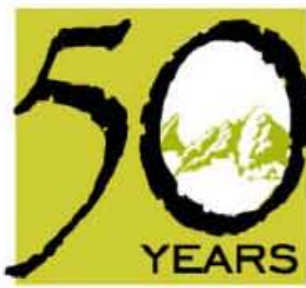

U.S. Department of Commerce

$1954-2004$

NISTIR 6618 and NTIA SP-04-416

\title{
Significant Papers from the First 50 Years of the Boulder Labs
}

Edited by

M.E. DeWeese M.A. Luebs

H.L. McCullough

\section{U.S. Department of Commerce Boulder Laboratories}

National Institute of Standards and Technology

National Oceanic and Atmospheric Administration
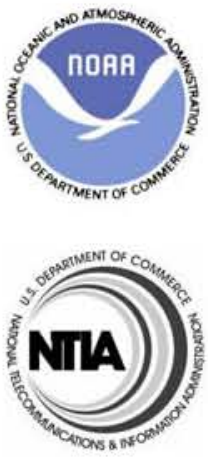


\section{Significant Papers from the First 50 Years of the Boulder Labs}

Edited by

M.E. DeWeese, NIST

M.A. Luebs, NTIA

H.L. McCullough, NOAA

Sponsored by

National Institute of Standards and Technology National Telecommunications and Information Administration National Oceanic and Atmospheric Administration Boulder, Colorado

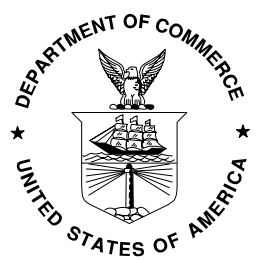

U.S. Department of Commerce Donald L. Evans, Secretary

National Institute of Standards and Technology Arden L. Bement, Jr., Director

National Oceanic and Atmospheric Administration Conrad C. Lautenbacher, Jr., Undersecretary of Commerce for Oceans and Atmosphere and NOAA Administrator 


\section{Acronym Definitions}

CEL CIRES

CRPL

CU

DOC

EDS

ERL

ESSA

ITS

ITSA

JILA

NBS

NCDC

NGDC

NIST

NOAA

NTIA

OAR

OT

OTP

RSMS

SEC
Cryogenic Engineering Laboratory Cooperative Institute for Research in Environmental Sciences Central Radio Propagation Laboratory University of Colorado

Department of Commerce

Environmental Data Service ESSA Research Laboratory Environmental Science Services Administration

Institute for Telecommunication Sciences Institute for Telecommunication Sciences and Aeronomy

Joint Institute for Laboratory Astrophysics

National Bureau of Standards National Climatic Data Center National Geophysical Data Center National Institute of Standards and Technology National Oceanic and Atmospheric Administration National Telecommunications and Information Administration

Office of Oceanic and Atmospheric Research Office of Telecommunications Office of Telecommunications Policy Radio Spectrum Measurement System Space Environment Center 


\section{Contents}

Page

Acronym Definitions ................................................................................... ii

Historical Publications Committee …............................................................ iv

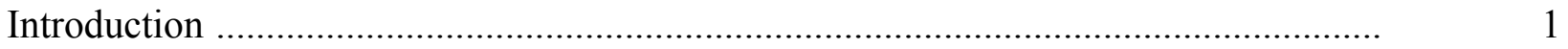

Historical Overview of the Federal Laboratories in Boulder ................................... 2

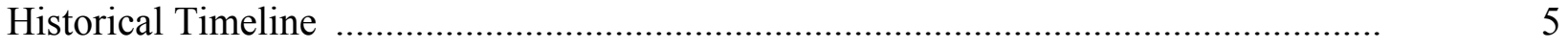

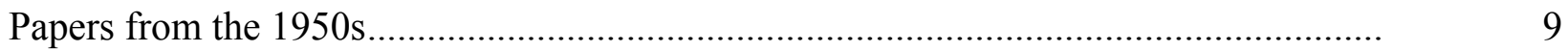

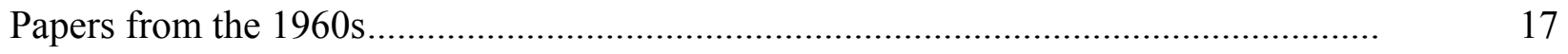

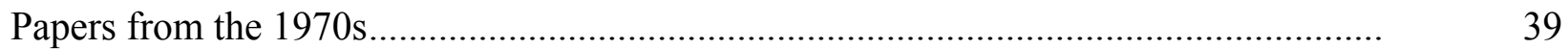

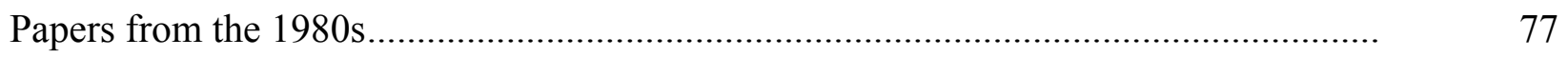

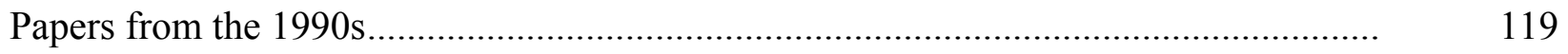

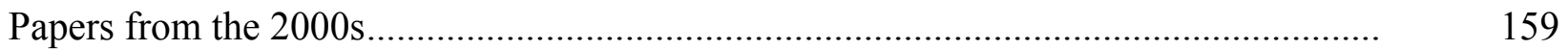

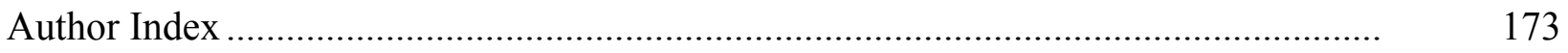

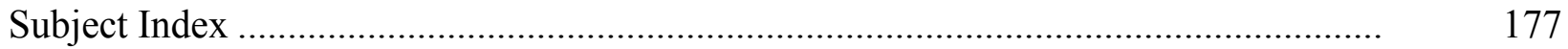




\title{
Boulder Labs 50th Anniversary Historical Publications Committee
}

\author{
Chair: Edie DeWeese, NIST \\ Debe Daily-Fisher, NOAA \\ Evie Gray, NTIA/ITS \\ Carol Knight, NOAA \\ Margaret Luebs, NTIA/ITS \\ Heather McCullough, NOAA \\ Kate Remley, NIST
}




\section{Introduction}

The Department of Commerce Boulder Labs were dedicated on September 14, 1954, in the shadow of World War II, and at the dawn of a bright future. This volume presents a snapshot of our research accomplishments in the half century since then. During this time, the Boulder Labs have evolved from the National Bureau of Standards' Central Radio Propagation Laboratory to today's National Institute of Standards and Technology, National Oceanic and Atmospheric Administration, and National Telecommunications and Information Administration's Institute for Telecommunication Sciences. The papers we have collected represent the most significant work of all the agencies of the Boulder Labs, whatever their name at the time of publication.

The past 50 years have been productive ones at the Boulder Labs. As you look through this collection of significant papers, think about the countless hours of work that brought them to being. Think, too, about the papers behind the papers: the works that provided the building blocks of the research represented here. Finally, think about the individual efforts of the staff members whose dedication has contributed so much to our community, the nation, and the world.

Edie DeWeese, Chair

Historical Publications Committee

Boulder, Colorado

September 2004 


\section{Historical Overview of the Federal Laboratories in Boulder}

In 1902, when Guglielmo Marconi observed from the SS Philadelphia that radio waves could be received at much greater distances at night than during the day, the foundation of a long history of joint radio and atmospheric research was laid. This interaction of research had ramifications for Boulder in the years to come.

Just months before Marconi realized this diurnal effect out in the Atlantic, the National Bureau of Standards (NBS) was established in Washington, DC, on March 3, 1901. Its mission was to establish the standards of measurement for the purposes of commerce. The Radio Laboratory Section was formed in 1913 and designated as Section 6 of the Electricity Division of NBS. This section moved to its new facility in October of 1918 far out on Connecticut Avenue in Northwest Washington, where it would remain until 1954.

On May 1, 1946, the Radio Laboratory Section was established as its own division within NBS and named the Central Radio Propagation Laboratory (CRPL). On that same day the Radio Propagation Executive Council held its first meeting and discussed the inadequate space available at the dated Connecticut Avenue campus. A new larger facility to house the new division was proposed at this location; however, CRPL staff realized that a new facility also required a quiet environment relatively free of radio interference. The existing campus could not provide such an environment.

In January of 1947, funding was approved for a new CRPL building at the NW Washington site with construction to begin in 1948. However, construction was postponed to consider a consolidated facility outside the Washington area. This site should accommodate the division as well as the test and field site, and ideally located near a suitable university with a relatively quiet radio environment. When President Truman issued a confidential policy in early 1949 directing that no more major government buildings be constructed in the District of Columbia, the search for a new location was on. This effort to decentralize the Federal government was a result of early cold war concerns of nuclear attacks on Washington.

In June of 1949, the director of NBS, Dr. Edward U. Condon, attended the Echo Lake Cosmic Ray Symposium at Mount Evans near Idaho Springs. Present at the Symposium was Dr. Walter Orr Roberts, Superintendent of the High Altitude Observatory, University of Colorado at Climax Springs. Dr. Roberts invited Dr. Condon to visit Boulder that weekend and take a tour of the CU campus. Dr. Roberts was convinced that Boulder was the ideal location for the new CRPL laboratory. Dr. Condon agreed and returned with several members of the CRPL staff to enjoy the 4th of July holiday in Boulder.

The 81 st Congress had authorized only $\$ 4,475,000$ in funding for the construction of the new radio laboratory. No appropriations were slated for the purchase of property. At the time, Charlottesville, Virginia, and Palo Alto, California, were under serious consideration for the location of the new laboratory because they had viable locations and land available. The Boulder Chamber of Commerce made a move to enter the competition. Led by its President, Vergyl H. 
Reynolds, and Junior Past President, John F. Allardice, the Chamber created the Standards Committee on February 27, 1950, in order to raise funds to purchase land. Two tracts of land totaling 217 acres were available south of town that could be obtained for approximately $\$ 70,000$. The land was situated west of Marshall Road (now South Broadway) and south of Green Mountain Cemetery.

On March 2, James J. Yeager, a Boulder businessman, accepted chairmanship of the campaign. By early April, Yeager had a large group of Boulder citizens organized for the Boulder Chamber of Commerce-U.S. Bureau of Standards Radio Laboratory Fund Campaign. The campaign officially kicked off at 7:30 a.m. on April 1 at a breakfast addressed by Elmore Peterson, Dean of the Business School. A short two weeks later, over $\$ 90,000$ was collected and the campaign was over. The largest contribution, totaling \$10,000, came from the Boulder Elks Lodge No. 566. All contributions of $\$ 100$ or more were recognized by public announcement and later by inscription on a metal plaque that was placed in the lobby entrance. The final negotiated price for the land was $\$ 63,000$, with the excess money used to purchase land east of town on Arapahoe Road. (This tract of land was known as the Boulder Industrial Park and was later occupied by the Ball Brothers Research Corporation.) On June 13, 1950, the deed to the land was transferred by the Boulder Chamber of Commerce to the United States of America.

The architectural firm of Pereira, Luckman, and Stanton was chosen to design the Radio building for the new property. This firm subsequently won the 1954 Award of Merit from the American Institute of Architects for their design. However, before construction of the Radio laboratory began, another important construction project began on the newly acquired property. On August 29, 1949, the Soviet Union detonated that country's first atomic bomb. The United States' response to this development was to accelerate research for the production of the super bomb, or hydrogen bomb. Initial designs called for copious amounts of liquefied hydrogen. No large liquifier facilities existed this side of the Mississippi, and none could supply the amounts required. Therefore, on March 28, 1951, it was announced that a large hydrogen liquefying plant would be constructed at the Boulder site. Construction was completed in a little over a year, an incredible feat of engineering. The new Cryogenic Engineering Laboratory was capable of producing 350 liters of liquid hydrogen an hour, and supplied the liquid used in the world's first hydrogen bomb. This bomb, code named Mike, was exploded on November 1, 1952, at Elugelab Island in the South Pacific. Part of the original building is still in use today, although subsequent hydrogen bomb tests did not require large amounts of liquid hydrogen.

About the time these large construction projects began, NBS negotiated for a temporary facility in Boulder. The Colorado National Guard Armory on Foothills Road (now North Broadway) was transferred to the CRPL. The staff would remain for over three years as the Radio building was constructed. The Radio building was constructed by the Olson Construction Company of Denver for $\$ 3,920,000$. Only four of the present six wings were built, for a gross area of approximately 172,800 square feet. The building was completed in March of 1954 with the people and equipment from the Armory site moving in April. By the time the dedication ceremonies were held in September, over 512 metric tons of equipment and over 450 people had moved to Boulder from Washington. The majority of the move was conducted in July during a recordbreaking heat wave of $104{ }^{\circ} \mathrm{F}$ in Boulder and up to $118{ }^{\circ} \mathrm{F}$ on the route across the mid-west. 
On September 14, 1954, President Eisenhower, the first incumbent President ever to visit Boulder, led the dedication ceremonies. With area schools called off, an estimated crowd of 10,000 citizens and dignitaries attended the ceremonies. Eisenhower declared "...it is my privilege to dedicate this facility of the National Bureau of Standards to the welfare of humanity - in America and throughout the world." With that he pushed a button and unveiled the metal plaque with the names of the Boulder contributors. Also unveiled was a slab of granite with the names of the architects and builders. Sealed behind the granite slab is a container with 15 important documents published by NBS.

Several more buildings were constructed in the sixties on the Bureau campus. All of the roads on the site were named after Nobel Prize winners in Physics. The roster of names included: Anderson, Appleton, Compton, Curie, Lawrence, Marconi, Millikan, and Wilson. Also constructed was the Joint Institute for Laboratory Astrophysics (JILA) building on the CU campus. JILA is a cooperative research facility between NIST and the University of Colorado, an association that produced its own Nobel Prize for Physics in 2001.

In the early sixties NBS acquired 1800 acres of land north of Boulder known as Table Mountain. With its large flat terrain, this site is still used as a radio field and test site. Other sites were also established at this time, including a $20 \mathrm{kHz}$ transmission site near the town of Sunset in Four Mile Canyon, an observation facility at Fritz Peak south of Rollinsville, and the WWV/WWVB broadcast site north of Ft. Collins.

On October 11, 1965, the Central Radio Propagation Laboratory was transferred from NBS to the United States Weather Bureau and became the Environmental Science Services Administration (ESSA), Institute for Telecommunication Sciences and Aeronomy (ITSA). With that transfer, the Boulder Laboratories, National Bureau of Standards was renamed the Boulder Laboratories, United States Department of Commerce. The ESSA Research Laboratory (ERL ) was created and with no facilities available, space was leased from CU.

On October 3, 1970, ESSA was renamed the National Oceanic and Atmospheric Administration (NOAA). This consolidated several groups into one agency to provide atmospheric, marine and oceanic services. In 1978 ITS joined NTIA, and on August 23, 1988 the National Bureau of Standards was renamed the National Institute of Standards and Technology (NIST). Through the years, these agencies have continued to grow and expand. On October 18 of 1996, ground was broken for a new NOAA building on the south part of the original 217-acre site that the citizens of Boulder had purchased 47 years before. Construction began on November 18, 1996, and the facility was dedicated on November 20, 1998. This facility houses 236,000 square feet of office and laboratory space at an estimated cost of $\$ 56$ million. The NOAA building consolidates the facilities that had been spread out around Boulder. 


\section{Boulder Labs Timeline}

1946: Central Radio Propagation Lab is organized to direct research and predictions in the field of radio wave propagation, including investigation of solar and geophysical effects on radio communication, and research on the upper atmosphere.

1950: In only two weeks, the people of Boulder raised $\$ 90,000$ to buy the 217 acres of land the Boulder Labs now occupies. This land donation was an important factor in the choice of Boulder for the Labs, along with its varied terrain, freedom from radio interference, and proximity to the University of Colorado.

1951: The first building is constructed - not to house radio research, but to serve as the world's largest cryogenic facility, able to produce up to 320 liters of liquid hydrogen per hour.

1951: The first CRPL staff were transferred to Boulder to work in the Colorado National Guard armory on North Broadway while construction was underway at the 325 Broadway site.

1954: On September $14^{\text {th }}$, President Dwight D. Eisenhower, the first incumbent President ever to visit Boulder, arrives to dedicate the Boulder Laboratories.

1954: The Bureau's first atomic clock, NBS-1, is built in Washington and shipped to Boulder. Using the oscillation of cesium atoms, it sets a new standard for the second, so accurate it lost only one second every 300 years.

1954-1965: CRPL develops worldwide ionospheric soundings, used to predict high-frequency usable frequencies for radio services, very low frequency (VLF) transmissions for reliable communication and navigation, as well as developing very high frequency (VHF) ionospheric scatter systems for transoceanic and Arctic links.

1956: Boulder's World Data Center system is established to collect, archive, and distribute crucial data for the first International Geophysical Year in 1957.

1957: Modern data record of atmospheric $\mathrm{CO}_{2}$, a major greenhouse gas, begins at Mauna Loa Observatory in Hawaii; measurements continue today under NOAA's Climate Monitoring and Diagnostics Laboratory.

1960s: ITSA's propagation research in the millimeter-wave bands (20 GHz and above) produces a body of knowledge now being used by new commercial wireless systems.

1962: A pulsed ruby laser is built by NBS's Donald Jennings, making possible Apollo 11's famous laser experiment measuring the Moon's distance from Earth.

1965: In 1965, CRPL is transferred from NBS to the newly formed Environmental Science Services Administration (ESSA), and renamed the Institute for Telecommunication Sciences and Aeronomy (ITSA). 
1965: Environmental Science Services Administration (ESSA), NOAA's forerunner, is created to encompass the Weather Bureau, Coast and Geodetic Survey, and the Central Radio Propagation Lab, now reorganized to form the Aeronomy Division, forerunner of NOAA's Aeronomy Laboratory, and the Space Environmental Forecasting Division, forerunner of NOAA's Space Environment Center.

1965: Institutes for Environmental Research established in ESSA to study atmospheric sciences, oceanography and earth sciences. The Institutes, predecessor of NOAA's Environmental Research Laboratories (ERL), are headquartered in Boulder.

1965: Environmental Data Service (EDS) formed within ESSA includes National Geophysical Data Center (NGDC), which collects, organizes, and disseminates data on the Earth and its systems, and continues under NOAA today.

1965: Space Disturbance Forecast Center begins routine forecasting service with USAF Air Weather Service, a relationships that continues today.

1965: Carl Disch, a Boulder Labs employee, disappears from an Ionosphere-Forward Scatter station in Byrd, Antarctica. He apparently missed a hand line during high winds and was never seen again.

1967: The new Joint Institute for Laboratory Astrophysics, JILA, is dedicated on the University of Colorado campus in Boulder. This opens up research projects in astrophysics, atomic physics, and aerodynamics, and is a cooperative effort between NIST and CU.

1967: NBS staff redefine the second, which had been based on a fraction of the mean solar day, as "the duration of 9,192,631,770 periods of radiation corresponding to the transition between the two nuclear hyperfine levels of the fundamental state of the atom of cesium 133."

1967: ITSA is split into four entities within ESSA, with three laboratories oriented toward environmental science issues, and the new Institute for Telecommunication Sciences (ITS) focused exclusively on telecommunications.

1967: NOAA's first joint institute, the Cooperative Institute for Research in Environmental Sciences, established at the University of Colorado to partner with the local labs in areas of atmospheric research.

1968: NIST scientists develop slush hydrogen, a promising and desirable rocket fuel.

1969: Apollo 11 leaves reflectors on the moon in order to more accurately measure the distance between Earth and the moon. JILA scientist James Faller suggested the idea, and NBS was able to accurately measure the distance to within $2.5 \mathrm{~cm}$. The reflectors are still used today to test gravitational and mass theories.

1972: NBS TvTime is demonstrated to show how subtitling can benefit the hearing impaired. It is later approved for mass use by the Federal Communications Commission and NBS wins an Emmy Award in 1980 for excellence in engineering. The NBS staff responsible for the work (Dick Davis, Jim Jespersen, and George Kamas) were invited to the White House to receive President Carter's congratulations. 
1972: Ken Evenson and his team are entered in The Guinness Book of World Records for measuring the frequency of laser light. It leads to a new definition of the meter that is 100 times more accurate than the previous standard.

1973: ITS designs the Radio Spectrum Measurement System (RSMS), a state-of-the-art computercontrolled measurement system in a mobile van.

1975: Aeronomy Lab develops the first Doppler radar wind profiler, located near the town of Sunset in the Boulder front range, for the measurement of winds up to 10 kilometers away. The Sunset radar pioneered wind-profiling technology that later involves other Boulder labs and results in improvements to daily weather forecasts and warnings.

1976: Based on its comprehensive measurements of man-made noise, ITS develops the CCIR / ITU$\mathrm{R}$ model still used today in radio link design.

1978: ITS becomes part of the National Telecommunications and Information Administration.

1979: Program for Regional Observing and Forecasting Systems (PROFS) is created in ERL to develop a weather forecast workstation for severe-storm warnings.

1980: ETL demonstrates first high-energy pulsed Doppler lidar (which uses optical rather than radio frequencies) for atmospheric wind measurements, deployed the next year to monitor winds during landing of the second space shuttle flight.

1986: Aeronomy Lab scientist proposes that the cause of the Antarctic ozone hole is the interaction of human-produced chlorine compounds with ice clouds in the upper atmosphere; scientists from NOAA's Aeronomy Lab and CMDL lead the first National Ozone Expedition to the South Pole that proves the theory correct, propelling international efforts to protect the ozone layer.

1987: Study of superconductivity continues, and research finds more uses for big magnets, such as magnetically levitated trains, and MRI imaging, which is widely used in medical science today.

1988: The name we came to Boulder with changes - the National Bureau of Standards becomes the National Institute of Standards and Technology.

1989: Staff at NIST build a 10 volt reference standard involving 14,184 Josephson junctions operating simultaneously.

1990: A new species of dinosaur — the only one named after a government agency—Drinker nisti, is discovered by Boulder NIST scientists and amateur paleontologists Jim Filla and Jim Siegwarth, along with CU professor Robert Bakker.

1991: NIST scientists Drullinger, Glaze, Lowe, and Shirley improve upon NIST-7, the international time standard, with new laser-line narrowing methods. The new methods increased both accuracy and stability for the atomic clock. 
1994: Developed by NOAA's Forecast Systems Laboratory, the Rapid Update Cycle (RUC) weather forecast model becomes operational at the National Weather Service as the first forecast model using isentropic coordinates in the vertical and high frequency data for aviation, severe weather, and general forecasts.

1995: ITS collaborates on a project to achieve land mobile radio (LMR) interoperability.

1995: Scientists at JILA discover a new form of matter, the Bose-Einstein condensate.

1996: FSL provides critical support for developing the core Advanced Weather Interactive Processing System (AWIPS) software, crucial to National Weather Service modernization across the country.

1997: Climate Diagnostics Center researchers contribute to advancing a new approach to predicting U.S. El Niño climate impacts that is applied to the 1997-1998 El Niño.

1998: Algorithms developed by ITS staff for estimating audio quality are adopted as an American National Standard.

1999: NIST-F1, the cesium fountain clock, is unveiled and is currently the most accurate clock in the world. It loses less than one second every 20 million years and ticks over 9 billion times per second.

1999: ETL conducts the first test of a buoy-mounted wind profiler, making possible better observations of conditions over the oceans, and leading to improved models for climate and weather forecasts.

2001: Eric Cornell and Carl Wieman of JILA, and Wolfgang Ketterle of MIT win the 2001 Nobel Prize for Physics for their discovery of the Bose-Einstein condensate.

2001: NIST celebrates its Centennial-The National Bureau of Standards was chartered by Congress on March 3, 1901.

2001: ITS-developed glossary of telecommunications terms, previously a Federal Standard, becomes an American National Standard.

2001: ITS characterizes ultrawideband (UWB) signals, which have been proposed as a partial solution to spectrum crowding. The results were used in a related study of the potential effects of UWB signals on GPS receivers.

2003: ITS Video Quality Metric software is adopted as an American National Standard.

2003: NOAA wind profiler network assists NASA in tracking debris from the space shuttle Columbia accident.

2003: NIST Materials Reliability staff analyze the U.S. Capitol Dome for repairs and participate in research on New York's World Trade Center disaster.

2004: NOAA scientist Susan Solomon wins the prestigious Blue Planet Prize for her work in identifying the cause of the Antarctic ozone hole. 


\section{Papers from the 1950s}

The constants in the equation for atmospheric refractive index at radio frequencies Ernest K. Smith, Jr., and Stanley Weintraub, 1953

Measurements of the phase of radio waves received over transmission paths with electrical lengths varying as a result of atmospheric turbulence

Jack W. Herbstreit and Moody C. Thompson, 1955

A refined X-band microwave microcalorimeter

Glenn F. Engen, 1959

Confidence intervals for the expectation of a Poisson variable

Edwin L. Crow and Robert S. Gardner, 1959

Cryogenic engineering

Russell B. Scott, 1959

System loss in radio wave propagation

Kenneth A. Norton, 1959

The riometer-A device for the continuous measurement of ionospheric absorption C. Gordon Little and Harold Leinbach, 1959 


\title{
The constants in the equation for atmospheric refractive index at radio frequencies
}

\author{
Ernest K. Smith, Jr. and Stanley Weintraub \\ NBS Central Radio Propagation Laboratory \\ Proceedings of the Institute of Radio Engineers, 41(8): 1035-1037, 1953
}

\section{Significance}

When Ernie Smith came out to Boulder in 1951, his first job with the Central Radio Propagation Laboratory (CRPL) was to be head of the Radio Meteorology group. Discovering that the thenaccepted constants for the atmospheric refractive index were seriously in error, he felt that it was the responsibility of NBS to improve them. He and Stanley Weintraub gathered together the existing experimental data on dry air and on water vapor and put together this report. Smith left Boulder in 1952 to finish his Ph.D. at Cornell (returning a few years later) and the paper was published in the Proceedings of the IRE. The constants have held up amazingly well over time.

\begin{abstract}
Recent improvements in microwave techniques have resulted in precise measurements which indicate that the conventional constants $K_{1}=79^{\circ} \mathrm{K} / \mathrm{mb}$ and $K_{2}{ }^{\prime}=4,800^{\circ} \mathrm{K}$ in the expression for the refractivity of air, $N=(n-1) 10^{6}=\left[K_{1} / \mathrm{T}\right]\left(p+K_{2}{ }^{\prime} e / T\right)$ should be revised. Various laboratories appear to have arrived at this conclusion independently. In much of radio propagation work the absolute value of the refractive index of the atmosphere is of small moment. However, in some work it is important and it seems highly desirable to decide upon a particular set of constant.
\end{abstract}

Through consideration of the various recent experiments this paper arrives at a relation

$$
N=\frac{77.6}{T}\left[p+4,810 \frac{e}{T}\right],
$$

where

$p=$ total pressure in millibars

$e=$ partial pressure of water vapor in millibars

$T=$ absolute temperature $={ }^{\circ} \mathrm{C}+273$

This expression is considered to be good to 0.5 per cent in $N$ for frequencies up to $30,000 \mathrm{mc}$ and normally encountered ranges of temperatures, pressure and humidity. 


\title{
Measurements of the phase of radio waves received over transmission paths with electrical lengths varying as a result of atmospheric turbulence
}

\author{
Jack W. Herbstreit and Moody C. Thompson \\ NBS Central Radio Propagation Laboratory \\ Proceedings of the Institute of Radio Engineers, 43(10): 1391-1401, October 1955
}

\section{Significance}

Jack Herbstreit, Chief of the Tropospheric Propagation Section of the CRPL, was the director of the Cheyenne Mountain tropospheric propagation experiments, which began in 1950, almost a year before the first CRPL employees arrived in Boulder. This paper, based on that project, looks at how atmospheric changes or discontinuities cause tropospheric scatter ("troposcatter") of radio waves. Troposcatter is the propagation of radio waves by scattering as a result of irregularities or discontinuities in the physical properties of the troposphere, such as humidity, temperature, and pressure, and temporal changes such as wind or other movement. The measurements described in this paper enabled researchers to apply atmospheric parameters to extend the range of communication far beyond "line of sight." Troposcatter was used as a method for transhorizon communications by the military beginning in the 1950s, most notably in the Distant Early Warning (DEW) line in northern Greenland, Canada, and Alaska, which was intended to warn against an over-the-pole invasion. These days troposcatter has been largely replaced by satellite communications; however, it is still used in some military applications, and in situations where relay stations are impractical.

\begin{abstract}
A system for the measurement of the variations in electrical lengths of radio propagation paths is described. The observed path-length instabilities are considered to be caused by the same atmospheric turbulence responsible for the existence of very high frequency and ultra high frequency fields far beyond the radio horizon. Results obtained on $172.8 \mathrm{mc}$ and 1,046 mc along $3 \frac{1}{2-}, 10-$, and 60 -mile paths are reported. It is pointed out that measurements of this type provide a powerful tool for the study of the size and intensity of the refractivity variations of the atmosphere giving rise to the observed phenomenon.
\end{abstract}




\title{
A refined $\mathrm{X}$-band microwave microcalorimeter
}

\author{
Glenn F. Engen \\ NBS Radio Standards Laboratory
}

NBS Journal of Research 63C(1): 77-82, July-September 1959

\section{Significance}

The paper describes the "Engen calorimeter" that appears in the Boulder Laboratories museum. This paper and a predecessor by Macpherson and Kerns of NBS in Washington, D.C., laid out basic principles of calorimeter design that are still applicable today. These design principles have been used at NIST and other national standards labs for over 40 years. A quick perusal of recent papers showed that at least four national measurement labs (China, Germany, Korea, and United Kingdom) have cited this paper since 1990, over 30 years after its original publication.

\begin{abstract}
The microcalorimetric method for evaluation of the efficiency and substitution error of a bolometer mount proposed by Macpherson and Kerns has been the object of further study and refinement at the Boulder Laboratories of the National Bureau of Standards, and an improved instrument based on this technique has been recently placed in operation.

The new microcalorimeter design features are: (1) Greatly improved ambient temperature control, permitting higher sensitivity and resolution; (2) improved d-c instrumentation; (3) improved mechanical construction giving better repeatability; (4) relocation of the thermopile so that it no longer is attached directly to the bolometer mount, thus providing flexibility in the choice of termination; and (5) a more comprehensive treatment of the calorimetric substitution or equivalence error. These features permit the determination of the effective efficiency of the bolometer mount to an accuracy better than 0.2 percent.
\end{abstract}




\title{
Confidence intervals for the expectation of a Poisson variable
}

\author{
Edwin L. Crow \\ NBS Central Radio Propagation Laboratory \\ Robert S. Gardner \\ U.S. Naval Ordnance Test Station, China Lake, California
}

Biometrika, 46: 441-453, 1959

\section{Significance}

This paper presents a fundamental advancement in mathematical theory and practice for calculating confidence intervals ("error bars") for the expectation of a Poisson-distributed random variable. The Poisson distribution is used to approximate the binomial distribution in situations where the number of "trials" is large and the probability of "success" on any individual trial is small. Such situations are commonly encountered in telecommunication performance measurement (e.g., measurement of bit or block error or loss ratios or blocking probabilities). Calculating confidence intervals can limit the duration and cost of such measurements. The technique described in this paper provides the narrowest possible confidence limits for measuring a Poisson random variable, and thus enables such measurements to be conducted with minimum measurement time and cost. Measurement methods based on this technique have been specified in national and international standards and are widely used in telecommunication performance assessment.

\begin{abstract}
A table of two-sided confidence intervals for the expectation of a Poisson variable is presented for confidence coefficients $0 \cdot 80,0 \cdot 90,0.95$, and 0.999 and all values of the variable from 0 to 300. We denote the Poisson variable by $c$ and its expectation by $m$ and plot them as rectangular coordinates, upper and lower confidence limits for $m$ being plotted vertically along with $c$. If the successive upper limits for $c=0,1,2, \ldots$ be joined by line segments and the lower limits likewise joined, a semi-infinite region, called the confidence belt, is formed between the upper and lower broken lines. The confidence belt tabulated herein is determined by the properties (i) that, of all confidence belts for the Poisson expectation with a given confidence coefficient which are based directly on the Poisson variable $c$, it is as narrow as possible if width is measured in the horizontal $c$-direction; and (ii) that, of all such equally narrow confidence belts, it is that one with the smallest possible upper confidence limits. The second condition serves to minimize the lengths of the confidence intervals satisfying (i) for small values of $c$ at the expense of inappreciable relative increase in length of larger values of $c$.
\end{abstract}




\title{
Cryogenic engineering
}

\author{
Russell B. Scott \\ NBS Cryogenic Engineering Laboratory \\ Princeton, New Jersey: Van Nostrand Company, 1959
}

\section{Significance}

This was the first book published on cryogenic engineering and was written by the former chief and founder of the Cryogenic Engineering Laboratory (CEL). In 1959, the book presented for the first time a written description of all the engineering principles and practices that are unique to the field of cryogenic engineering. Most of the material presented in this book summarizes the results from the extensive studies carried out at the CEL of the NBS Boulder Laboratories from its founding in 1952 up to about 1958. Thus, there is much original work as well as a wonderful summary of nearly all that is important for cryogenic engineering. Even today, in 2004, researchers refer to this book quite often as a major source of information on cryogenic engineering. This monograph, since its publication, helped put the Boulder CEL on the map.

\section{Table of Contents}

\section{Introduction}

II. Liquefaction of Gases

III. Separation of Gases

IV. Cooling by Adiabatic Demagnetization

V. Low-Temperature Thermometry

VI. Insulation

VII. Storing and Transporting Liquefied Gases

VIII. Transfer of Liquefied Gases

IX. Properties of Cryogenic Fluids

X. Low-Temperature Properties of Structural Materials 


\title{
System loss in radio wave propagation
}

\author{
Kenneth A. Norton \\ NBS Central Radio Propagation Laboratory \\ NBS Journal of Research, 63D(1): 53-73, August 1959
}

\section{Significance}

Kenneth Norton developed the concept of transmission loss in describing the characteristics of tropospheric radio propagation. In this paper, he provided a full set of definitions for system, transmission, basic transmission, path antenna gain, and related concepts. These define the boundary between antenna and propagation path, and are fundamental to all propagation modeling. Norton also showed that by going to power (instead of field strength) for signal and noise, one can use a link budget and readily see the gains and losses in a system. Norton's work on transmission loss led to one of CRPL's most famous publications, Tech Note 101 (see Longley et al. 1965, this volume).

\begin{abstract}
A summary is presented of the ways in which the concept of system loss and the closely related concepts of transmission loss, basic transmission loss, propagation loss, and path antenna gain may be used for precise, yet simple, descriptions of some of the characteristics of radio wave propagation which are important in the design of radio systems. Definitions of various terms associated with the concept of system loss are given which introduce a greater flexibility into its use without any loss in precision. It is shown that the use of these added terms and concepts makes feasible the extension of the use of this method of description to any portion of the radio spectrum. A more general formula for the system loss is given which may be used for antennas with an arbitrarily small separation. Using this formula it is shown that the system loss between small electric or magnetic dipoles separated by a distance $\mathrm{d}$ « $\lambda$ can be made arbitrarily small even though the individual antennas have large circuit losses. Formulas are developed for the percentage of time that a desired signal is free of interference, and these are used to demonstrate methods for the efficient use of the spectrum. In particular, contrary to general belief, it is shown that efficiency is promoted by the use of high power and high antennas and, in the case of a broadcast service, sufficiently small separations so that there is appreciable mutual interference. An analysis is made of the variance of the path antenna gain in ionospheric scatter propagation. Methods are given for the calculation of the transmission loss for the ground wave and tropospheric scatter modes of propagation through a turbulent model atmosphere with an exponential gradient. Examples of such calculations are given which cover a wide range of frequencies and antenna heights. Finally, examples are given of the expected range of various tropospheric point-to-point scatter systems such as an FM multichannel teletype system, a television relay or an FM broadcast relay.
\end{abstract}




\title{
The riometer: A device for the continuous measurement of ionospheric absorption
}

C. Gordon Little

NBS Central Radio Propagation Laboratory

Harold Leinbach

Geophysical Institute, University of Alaska

Proceedings of the Institute of Radio Engineers, 47, 315-320, 1959

\section{Significance}

The riometer (relative ionospheric opacity meter) measures the intensity of wideband noise that impinges on the earth from deep space. The equipment consists of a stable receiver, a suitable antenna, a recorder, and a noise diode antenna. Ionospheric absorption had heretofore been very difficult to measure, so this method was a real boon to ionospheric physics. It is still being used for studies of the ionosphere, especially regarding solar flares and geomagnetic storms.

\begin{abstract}
A sensitive, self-balancing, noise-measuring equipment, known as the riometer, is described. This instrument has been designed for routine measurement of ionospheric absorption during the IGY, using the cosmic noise method. Application of this technique in the auroral zone has resulted in quantitative measurements of ionospheric absorption, even during polar blackouts.

The riometer has the advantages over a simple total-power cosmic noise receiving system of (1) linear response to changes of input noise power, (2) high accuracy in the presence of narrowband RF interference, and (3) good long-term stability.
\end{abstract}




\section{Papers from the 1960s}

A comparison of atomic beam frequency standards

Roger E. Beehler, Richard C. Mockler, and Charles S. Snider, 1960

Advances in cryogenic engineering, Vol. 1, Proceedings of the 1954 Cryogenic

Engineering Conference

Klaus D. Timmerhaus, 1960

Apparatus for determination of pressure-density-temperature relations and specific heats of hydrogen to 350 atmospheres at temperatures above $14 \mathrm{~K}$

Robert D. Goodwin, 1961

Electromagnetic waves in stratified media

James R. Wait, 1962

A survey of ionospheric effects upon earth-space radio propagation

Robert S. Lawrence, C. Gordon Little, and Hugh J.A. Chivers, 1964

Characteristics of the earth-ionosphere waveguide for VLF radio waves

James R. Wait and Kenneth P. Spies, 1964

Radio propagation by reflection from meteor trails

George R. Sugar, 1964

Wave hop theory of long distance propagation of low-frequency radio waves

Leslie A. Berry, 1964

Ionospheric radio propagation

Kenneth Davies, 1965

Transmission loss predictions for tropospheric communication circuits

Philip L. Rice, Anita G. Longley, Kenneth A. Norton, and Albrecht P. Barsis, 1965

Measuring oscillator and clock performance:

1. Atomic timekeeping and the statistics of precision signal generators

James A. Barnes, 1966

2. Statistics of atomic frequency standards

David W. Allan, 1966

Calorimetric measurement of pulsed laser output energy

Donald A. Jennings, 1966 
Radio meteorology

Bradford R. Bean and Evan J. Dutton, 1966

Thermal-energy associative-detachment reactions of negative ions

Fred C. Fehsenfeld, Eldon E. Ferguson, and Arthur L. Schmeltekopf, 1966

Thermodynamic properties of $\mathrm{He}^{3}-\mathrm{He}^{4}$ solutions with applications to the $\mathrm{He}^{3}-\mathrm{He}^{4}$ dilution refrigerator

Ray Radebaugh, 1967

Development and operation of a specialized technical information and data center (the Cryogenic Data Center)

Victor J. Johnson, 1968

Prediction of tropospheric radio transmission loss over irregular terrain:

A computer method

Anita G. Longley and Philip L. Rice, July 1968

Acoustic methods for remote probing of the lower atmosphere

C. Gordon Little, 1969 


\title{
A comparison of atomic beam frequency standards
}

\author{
Roger E. Beehler, Richard C. Mockler, and Charles S. Snider \\ NBS Time and Frequency Division
}

Nature 187: 681-682, 1960

\section{Significance}

This paper is primarily of interest for historical reasons, describing the atomic clock (NBS 2) that became in 1960 the first U.S. atomic standard for time and frequency. The clock was not only a substantial improvement in accuracy for measuring time and frequency, but also the first standard based on fundamental properties of matter rather than an artifact (such as the standard kilogram or a complex device such as the array of quartz oscillators that previously served as the national standard for time and frequency).

NBS created the world's first atomic clock in 1949 in Washington, D.C., based on transitions in ammonia molecules. In 1952, NBS adapted the science and technology to beams of cesium atoms in NBS 1, its first atomic clock intended as a standard. However, NBS 1 was not sufficiently reliable to serve as an operational standard. The U.S. national standard for time and frequency remained an ensemble of quartz oscillators. In 1954, the atomic clock program was transferred to the new Boulder Laboratories. The Boulder Labs developed NBS 2, which had electronic servo-control that enabled the atomic clock for the first time to be operated reliably and automatically. On January 1, 1960, NBS 2 was officially installed as the nation's time and frequency standard. The paper reports the comparison of NBS 1 and NBS 2, demonstrating an uncertainty of about 1.5 parts in $10^{11}$, equivalent to about 1 second in 2,000 years. Since that time, NBS/NIST has improved the performance of atomic clocks by a factor of about 10 per decade, with the current NIST-F1 laser-cooled atomic fountain clock having an accuracy of about 1 part in $10^{15}$, equivalent to about 1 second in 30 million years. This continual improvement has led to transforming commercial applications, such as the Global Positioning System and cellular communications networks, and to new scientific applications such as precision tests of quantum mechanics, relativity, and astrophysics. Atomic clocks quickly became so accurate that it became clear the rotation of the earth changed by small amounts in unpredictable ways. Thus by international agreement in 1967, the second was redefined in terms of the cesium atomic clock transition, replacing the definition based on the rotation of the earth. Thanks to continuing advances in atomic clocks, the second is by far the most accurately realized of the seven fundamental units. 


\title{
Advances in cryogenic engineering, Vol. 1, Proceedings of the 1954 Cryogenic Engineering Conference
}

\author{
Klaus D. Timmerhaus, Editor \\ NBS Cryogenic Engineering Laboratory and \\ University of Colorado
}

New York: Plenum Press, 1960

\section{Significance}

This first conference on cryogenic engineering was organized by NBS and started what has become the biennial Cryogenic Engineering Conference (CEC) that when combined with the Cryogenic Materials Conference attracts about 800 to 900 participants from around the world. It is the largest conference in the field of cryogenic engineering. Klaus Timmerhaus was a professor in the Chemical Engineering Department at the University of Colorado, but was also employed at NBS as a part time employee for many years.

Abstract (since the book has no abstract, an excerpt from the Foreword is included)

... Cryogenic engineering is concerned with developing and improving low temperature processes, techniques, and equipment; determining the physical properties of structural and related materials used in producing, maintaining, and using low temperatures; and the practical application of low temperature techniques and processes. These low temperatures are below those usually encountered in refrigerating engineering. It is rather difficult to assign a definite temperature which serves to divide refrigerating engineering from cryogenic engineering. A temperature below $-150^{\circ} \mathrm{C}$, however, is generally associated with cryogenic engineering.

There is ample reason for treating cryogenic engineering as a special field of engineering. The physical properties of materials at very low temperatures differ so markedly from those commonly encountered that the engineer cannot rely on his ordinary experience. Because of the rapid growth of this field of engineering in the past ten to fifteen years, many new and highly complex engineering problems have arisen in the low temperature field. In order to improve the interchange of information among organizations active in cryogenic engineering, the National Bureau of Standards-Atomic Energy Commission Cryogenic Engineering Laboratory in Boulder, Colorado, sponsored the first Cryogenic Engineering Conference on September 8, 9, and 10, 1954, at the National Bureau of Standards Boulder Laboratories... . 


\title{
Apparatus for determination of pressure-density-temperature relations and specific heats of hydrogen to 350 atmospheres at temperatures above $14 \mathrm{~K}$
}

\author{
Robert D. Goodwin \\ NBS Cryogenic Engineering Laboratory
}

NBS Journal of Research 65C(4): 231-243, 1961

\section{Significance}

The determination of the relation among temperature, pressure, and density is a key problem in establishing the important thermodynamic properties of a fluid. The apparatus described in this paper remains as a near state-of-the-art system for wide ranging measurements, and its capabilities have been extended through automation and an increase in the temperature range. The instrument has been used nearly continuously for more than 40 years to measure PVT relations and specific heats of numerous fluids, and the apparatus can be considered the heart and soul of the fluid property measurement program in Boulder. The early measurements on hydrogen described here form the basis of current standards on hydrogen properties (of keen interest for the "hydrogen economy"), and the uncertainty assessment described for the apparatus remains an exemplary protocol for density metrology.

\begin{abstract}
Method and apparatus are designed for more rapid determination of accurate, closely spaced, PVT and specific heat data than realized by previous procedures. A sequence of pressuretemperature observations at nearly constant density is made by a modified Reichsanstalt method. Temperatures of the essentially adiabatic piezometer are regulated by electric heating under control of the measuring thermometer. Instruments for measurement and control are integrated with a high-pressure calorimeter for compressed liquid and fluid. Calorimetric experimentation is accelerated by use of an electronic battery for the calorimetric heat supply and of a d-c power regulator developed for automatic shield control. Details are given of the PVT calibrations, adjustment computations, and comparisons with independent data.
\end{abstract}




\title{
Electromagnetic waves in stratified media
}

\author{
James R. Wait \\ NBS Central Radio Propagation Laboratory, and \\ University of Colorado
}

New York: Pergamon Press, 1962 (revised 1970)

\section{Significance}

This book is based on fundamental research in electromagnetic wave propagation that Wait performed in the CRPL from 1956 to 1962. The mathematical formulations are very general, and the "stratified media" models are applicable to the earth crust, troposphere, and ionosphere. The frequencies of the communication, navigation, and remote sensing applications range from extremely low frequencies to microwaves. The propagation theories in the book have served to guide experimental and theoretical tropospheric and ionospheric propagation programs both within the Boulder Labs and worldwide for over three decades. Cited over 1500 times, it was reissued by IEEE Press in 1996.

\begin{abstract}
This book is concerned with electromagnetic waves in media whose properties vary in one particular direction. The variation may consist of abrupt or continuous changes. In this sense the media may be generally classified as stratified. This is an idealization of many situations which occur in nature. For example, there is a tendency for our terrestrial atmosphere to occur in horizontal layers. Similarly, the properties of the earth's crust do not vary significantly in the horizontal direction. Consequently, a proper understanding of wave phenomena in stratified media is of great practical importance. The major theoretical task is to find solutions of Maxwell's field equations which satisfy the appropriate conditions imposed by the various boundary conditions.

Rather than present a sterile and formal treatment couched in the language of the mathematician, a physical approach is adopted. Furthermore, applications to the real world are used as illustrations of the theoretical principles. Special emphasis is given to radio waves in the frequency range from 3 to $30 \mathrm{kc} / \mathrm{s}$ which is described as v.l.f. (very low frequency). These frequencies correspond to wavelengths ranging from 100 to $10 \mathrm{~km}$. In such cases the ionized layers in the upper atmosphere do indeed behave as stratified media since the scale of the irregularities is usually small compared with the wavelength. In addition, many comparisons are made with experimental data on v.l.f. radio transmissions since only in this way can we ascertain if the adopted theoretical model has any real significance. An important feature of these waves is their low attenuation which enables communications at global distances. In addition, they exhibit remarkably stable phase characteristics... .
\end{abstract}




\title{
A survey of ionospheric effects upon earth-space radio propagation
}

\author{
Robert S. Lawrence, C. Gordon Little, and Hugh J.A. Chivers \\ NBS Central Radio Propagation Laboratory
}

Proceedings of the Institute of Radio Engineers, 47, 315-320, 1964

\section{Significance}

This paper was an attempt by the CRPL to bring some order and understanding to what was then the new field of earth-space radio propagation. It was the most accurate and authoritative text on the subject at the time, and was considered by the Laboratory to have put to rest various questions regarding Faraday rotation, group and phase delay, total electron content and ionospheric absorption of radio waves transiting the ionosphere. Advances in high power, high frequency transmission have made satellite communications much less difficult, but the work in this paper remains useful to this day.

\begin{abstract}
The frequency dependence is derived and the order of magnitude is presented for various ionospheric effects upon radio waves which have frequencies greater than the penetration frequency of the ionosphere. Among the phenomena considered are phase-path length change, refraction, frequency change, group-path delay, polarization rotation, and absorption. A detailed discussion is given of the mean value and variability of ionospheric absorption, refraction, scintillations, and polarization changes.
\end{abstract}




\title{
Characteristics of the earth-ionosphere waveguide for VLF radio waves
}

\author{
James R. Wait and Kenneth P. Spies \\ NBS Central Radio Propagation Laboratory
}

NBS Technical Note 300, December 30, 1964

\section{Significance}

Dr. Wait came up with the idea of modeling the propagation of VLF radio waves, used in military applications, by thinking of the space between the earth and the ionosphere as a kind of giant "waveguide" for the extremely long VLF radio waves. A waveguide is a device, usually a hollow round or rectangular tube, used to trap radio waves and guide them internally to another location. This Technical Note included both the mathematics for VLF earth-ionosphere waveguide propagation and extensive numerical results for frequencies from 8 to $30 \mathrm{kHz}$. In the days before fast computers, this was the first publication to present extensive numerical results for the relevant propagation parameters (attenuation rate, phase velocity, and field distribution) for a realistic curved earth and layered ionosphere model. The graphical results that are included were the only ones available for many years, and as a result this publication received an unusually large number of citations in the literature.

\begin{abstract}
The principal results of this technical note are graphical presentations of the attenuation rates, phase velocities, and excitation factors for the dominant modes in the earth-ionosphere waveguide. The frequency range considered is $8 \mathrm{kc} / \mathrm{s}$ to $30 \mathrm{kc} / \mathrm{s}$. The model adopted for the ionosphere has an exponential variation for both the electron density and the collision frequency, and the effect of the earth's magnetic field is considered. Comparison with published experimental data confirms that the minimum attenuation of VLF radio waves in daytime is approximately at $18 \mathrm{kc} / \mathrm{s}$, while at night it is somewhat lower. The directional dependences of propagation predicted by the theory are also confirmed by experimental data.
\end{abstract}




\title{
Radio propagation by reflection from meteor trails
}

\author{
George R. Sugar \\ NBS Central Radio Propagation Laboratory
}

Proceedings of the Institute of Electrical and Electronics Engineers, 52(2): 116-136, 1964

\section{Significance}

In the early 1960s George Sugar developed one of the earliest examples of packet transmission. As meteors travel through the ionosphere they leave a column of ions which, when the aspect ratio is just right, allow a few seconds of radio transmission at frequencies well above the normal critical frequency of the ionosphere. The trick was to send a burst of signal during that brief period of transmission, then wait for the next favorable meteor trail to send the next. Sugar's paper, as well as his system, pioneered a new mode of propagation. This classic paper on the interaction of radio waves and meteors has recently been rediscovered due to renewed interest in meteor burst communications as an inexpensive (and now much more feasible) tool for longdistance communication.

\begin{abstract}
This paper is a survey of those characteristics of meteors, and of meteor propagation, which are important to the understanding and use of meteor ionization insofar as it provides a means of radio transmission. The subjects discussed include the utility of meteor bursts for intermittent radio communication, physical properties of meteors and meteor trails, reflection properties of individual trails, short-term statistical characteristics of received signals, and long-term statistical characteristics of received signals.
\end{abstract}




\title{
Wave hop theory of long distance propagation of low-frequency radio waves
}

\author{
Leslie A. Berry \\ NBS Central Radio Propagation Laboratory
}

Radio Science Journal of Research NBS/USNC-URSI, 68D(12): 1275-1284, December 1964

\section{Significance}

This paper provides a practical method of calculating field strength for propagation of lowfrequency radio waves over long distances in the LF and VLF frequency bands, when the propagation distances are well into the shadow region (the region beyond the radio horizon where radio waves must bend around the Earth). LF and VLF waves were and still are important for certain types of long distance communications, e.g., with submarines. The method presented in the paper is a simple physical interpretation of a very complex process, and represents a breakthrough in computation techniques for wave-hop propagation. The author demonstrates that this technique is valid not only for the homogeneous case, but also for the nonhomogeneous anisotropic case with only a small error. The characteristics of the propagation path that determine its delay include ground conductivity, distance, earth curvature, and height of reflection. The innovative computation theory presented in the paper was an important contribution to the scientific community at the time it was written.

\begin{abstract}
The idea that long radio waves propagating between the earth and ionosphere via discrete hops can be extended far into the shadow region by evaluating a series of complex integrals is exploited in this paper. Illustrative calculations of LF and VLF hops and total fields are shown as a function of distance. The second and higher hops show a pseudo-Brewster angle just before the caustic and attenuate like groundwaves in the shadow region.

The form of the series of integrals for an anisotropic ionosphere is given, and a model anisotropic ionosphere varying with height is used in a sample calculation.

Only a small error is caused by writing each hop as the product of an effective ionospheric reflection coefficient and an integral which is a function only of the other characteristics of the path.
\end{abstract}




\title{
Ionospheric radio propagation
}

\author{
Kenneth Davies \\ NBS Central Radio Propagation Laboratory
}

NBS Monograph 80, 1965 (Revised, updated, and reissued as Ionospheric Radio, IEE Electromagnetic Waves Series 31, Peter Peregrinus, Ltd., 1989)

\section{Significance}

The main function of the CRPL when it was formed in 1947 was the prediction of HF ionospheric propagation. Knowledge of how the ionosphere affects propagation was vital in radio communications. An early publication, NBS Circular 462, became a worldwide handbook. In 1957, the revision of this handbook was assigned to Ken Davies, a new employee. The result, which incorporates radio propagation research performed in Boulder during the 1950s and 1960s, was NBS Monograph 80, reprinted in 1966 by Dover. This excellent, broad-based text is notable for its clarity and accessibility.

For practitioners of HF communications and surveillance, Davies' books have been an invaluable reference. In fact, until the 1990s, Davies' books were the only sources for information about the practical aspects of radio wave propagation within and through the ionosphere. Most recent texts on HF communications and encyclopedia articles on the ionosphere refer to Monograph 80 and its later edition. Davies' books have been of value in many civilian and defense system developments, and in the evaluation of a number of earthspace and terrestrial communication concepts. This book set out to bridge the gulf between the pure scientific understanding of the ionosphere and the real world of commercial radio communication. Its success is demonstrated by the fact that some 40 years after the first edition, this text is still used by scientists and engineers. The original edition has had nearly 90 citations since 1975 and continues to be referenced now.

\begin{abstract}
There is not an abstract associated with the 1965 Monograph. However, the flyleaf of the 1989 edition sums up the intent and nature of the earlier work as well as the more recent. "Ionospheric Radio replaces an earlier publication, Ionospheric Radio Propagation, and is aimed at professional scientists, engineers, and students who need an intermediate-level reference and/or text. Students of aeronomy and radio wave propagation are introduced to basic wave theory in absorbing, anisotropic and dispersive media and to the physics of the production, loss, and movement of plasma in the ionosphere in the presence of the geomagnetic field. Various radio techniques are described and applications to the interpretation of ionospheric data to radio communications problems are presented. Topics include Earth-space propagation, ionospheric modification, ionospheric disturbances, propagation by scattering from plasma irregularities, topside sounding, and propagation on frequencies from the extremely low to the super high."
\end{abstract}




\title{
Transmission loss predictions for tropospheric communication circuits
}

\author{
Philip L. Rice, Anita G. Longley, Kenneth A. Norton, and Albrecht P. Barsis \\ NBS Central Radio Propagation Laboratory*
}

*Note: the 1967 revision, which is the definitive version, lists their affiliation as Institute for

Telecommunication Sciences and Aeronomy, Environmental Science Services Administration

NBS Technical Note 101, May 7, 1965 (revised May 1, 1966, and January 1, 1967)

\section{Significance}

The detailed point-to-point methods described in "Tech Note 101" established the basic framework for nearly all subsequent practical radio propagation prediction models, including the famous Longley-Rice model (also known as the Irregular Terrain Model). These methods serve as the basis for making quantitative estimates of radio propagation that, for example, determine how well a proposed radio system will meet the requirements for satisfactory service, free from harmful interference, thereby promoting more efficient use of the radio frequency spectrum. Nothing else has the breadth of this publication, which is why it is still very much sought after today.

\begin{abstract}
This report represents comprehensive methods for predicting cumulative distributions of transmission loss for a wide range of radio frequencies over any type of terrain and in several climatic regions. Such quantitative estimates of propagation characteristics help to determine how well proposed radio systems will meet requirements for satisfactory service, free from harmful interference. Thus they should provide an important step toward more efficient use of the radio frequency spectrum.
\end{abstract}

The need for comprehensive and accurate prediction methods is clearly demonstrated when measured transmission loss data for a large number of radio paths are shown as a function of path length. In figures I.1 to I.4 of annex I, long-term median values of attenuation relative to free space for more than 750 radio paths are plotted versus distance. The extremely wide scatter of these data is due mainly to path-to-path differences in terrain profiles and effective antenna heights. Values recorded for a long period of time over a single path show comparable ranges, sometimes exceeding 100 decibels. Such tremendous path-to-path and time variations must be carefully considered, particularly in cases of possible interference between co-channel or adjacent-channel systems.

The detailed point-to-point prediction methods described here depend on propagation path geometry, atmospheric refractivity near the surface of the earth, and specified characteristics of antenna directivity. They have been tested against measurements in the radio frequency range of 40 to $10,000 \mathrm{MHz}$ (megacycles per second)... . 


\section{Measuring oscillator and clock performance:}

\section{Atomic timekeeping and the statistics of precision signal generators}

James A. Barnes

NBS Time and Frequency Division

Proceedings of the Institute of Electrical and Electronics Engineers, 54: 207-220, February 1, 1966

\section{Statistics of atomic frequency standards}

David W. Allan

NBS Time and Frequency Division

Proceedings of the Institute of Electrical and Electronics Engineers, 54: 221-231, February 1, 1966

\section{Significance}

These two papers, appearing back-to-back in the same journal, build on each other to describe a crucial new statistical method to evaluate the performance of oscillators and timekeeping devices. This new method rapidly became universally used to measure oscillator and clock performance, and users quickly began calling it the "Allan variance" in recognition of one of the NBS scientists who developed the method.

NBS scientists James Barnes and David Allan recognized that standard statistics such as the mean and standard deviation were woefully inadequate to measure the performance of oscillators (such as quartz crystals, atomic clocks, etc.) because the noise in the systems depended on the frequency and/or observation time itself. Barnes and Allan conducted experiments and theoretical analyses to develop a new statistic based on second-difference methods which proved extremely useful for specifying the performance of oscillators and clocks, by frequency or by time of observation. This statistic became known as the "Allan variance" (although it probably should be called the "Allan-Barnes variance") and is now universally used by equipment manufacturers, test engineers, and researchers to specify the performance of oscillators. The statistic enables not just an overall specification of performance, but also in many cases identification of the different causes of noise in the system under test. Researchers at NBS/NIST and elsewhere expanded on the concept of the original Allan variance to develop a number of other closely related statistics which are also used to measure particular aspects of oscillator and system performance. Major users of the Allan variance and its close relatives include the telecommunications industry, defense applications, broadcasters, surveillance applications (radar, etc.), aerospace industry, and the time and frequency research community. 


\begin{abstract}
1
Since most systems that generate atomic time employ quartz crystal oscillators to improve reliability, it is essential to determine the effect on the precision of time measurements that these oscillators introduce. A detailed analysis of the calibration procedure shows that the third finite difference of the phase is closely related to the clock errors. It was also found, in agreement with others, that quartz crystal oscillators exhibit a "flicker" or $|\omega|^{-1}$ type of noise modulating the frequency of the oscillator.

The method of finite differences of the phase is shown to be a powerful means of classifying the statistical fluctuations of the phase and frequency for signal generators in general. By employing finite differences it is possible to avoid divergences normally associated with flicker noise spectra. Analysis of several cesium beam frequency standards have shown a complete lack of the $|\omega|^{-1}$ type of noise modulation.
\end{abstract}

\title{
Abstract 2
}

A theoretical development is presented which results in a relationship between the expectation value of the standard deviation of the frequency fluctuations for any finite number of data samples and the infinite time average value of the standard deviation, which provides an invariant measure of an important quality factor of a frequency standard. A practical and straightforward method of determining the power spectral density of the frequency fluctuations from the variance of the frequency fluctuations, the sampling time, the number of samples taken, and the dependence on system bandwidth is also developed. Additional insight is also given into some of the problems that arise from the presence of "flicker noise" (spectrum proportional to $\left.|\omega|^{-1}\right)$ modulation of the frequency of an oscillator.

The theory is applied in classifying the types of noise on the signals of frequency standards made available at NBS, Boulder Laboratories, such as: masers (both $\mathrm{H}$ and $\mathrm{N}^{15} \mathrm{H}_{3}$ ), the cesium beam frequency standard employed as the U.S. Frequency Standard, and rubidium gas cells.

"Flicker noise" frequency modulation was not observed on the signals of masers for sampling times ranging from 0.1 second to 4 hours. In a comparison between the NBS hydrogen maser and the NBS III cesium beam, uncorrelated random noise was observed on the frequency fluctuations for sampling times extending to 4 hours; the fractional standard deviations of the frequency fluctuations were as low as 5 parts in $10^{14}$. 


\title{
Calorimetric measurement of pulsed laser output energy
}

\author{
Donald A. Jennings \\ NBS Radio Standards Physics Division
}

IEEE Transactions on Instrumentation and Measurement, IM-15: 161-164, 1966

\section{Significance}

This paper reports the development of the first primary standard for laser energy measurements. NBS was the first national measurement institute to have such a standard, and provided the first traceable calibrations beginning in 1967.

\begin{abstract}
There are several methods by which one may measure the energy output of the pulsed laser. However, the technique which seems to be most promising as far as accuracy and precision are concerned is the calorimetric method. We have designed, built, and calibrated calorimeters for measuring the output energy of the pulsed ruby laser (6943 $\AA$ ). The heart of the calorimeter is a small absorption cell containing an aqueous solution of $\mathrm{CuSO}_{4}$. The temperature of the absorption cell, as measured by a thermocouple, indicates the energy absorbed by the calorimeter. The calorimeter was calibrated in two different ways: (1) the known heat capacity of the absorption cell and the thermocouple sensitivity calibration gives a calorimeter calibration, which agrees within 0.3 percent of (2) an electrical energy substitution calibration which is obtained via a heater wire contained in the absorption cell solution. A method has been devised by which two calorimeters may be intercompared. Calorimeters which we have built and calibrated agree with each other to about 0.7 percent. This specific calorimeter has been designed to measure energies up to $30 \mathrm{~J}$ and will take peak powers of up to $200 \mathrm{MW} / \mathrm{cm}^{2}$.
\end{abstract}




\title{
Radio meteorology
}

\author{
Bradford R. Bean and Evan J. Dutton \\ NBS Central Radio Propagation Laboratory
}

NBS Monograph 92, March 1, 1966

\section{Significance}

Radio Meteorology, published by NBS in 1966 and republished by Dover in 1968, is a compilation of work performed by the Central Radio Propagation Laboratory in radio meteorology during the previous decade. It is one of very few treatments of this subject, certainly the only one that goes into this much depth. Its focus is on the refraction, or bending, of radio waves by the atmosphere, under various meteorological conditions. Because the radio refractive index of air is a function of atmospheric pressure, temperature, and humidity, radio signals vary according to the time of year and the climate. They may also be attenuated by atmospheric gases and weather phenomena such as clouds, rain, and fog. Therefore, information in this monograph enables engineers to predict whether their communications systems will function properly in a specific place under specific conditions at a specific time of year. Radio Meteorology has been translated into several languages and is considered the "bible" on the subject, with maps from this volume showing up in various ITU Recommendations. The two editions have a total of 260 citations in the Science Citation Index since 1975.

\begin{abstract}
This volume brings together the work done in radio meteorology over the past ten years at the National Bureau of Standards' Central Radio Propagation Laboratory (CRPL). This decade has seen the development, on an international scale, of great emphasis upon the effects of the lower atmosphere on the propagation of radio waves. The CRPL group has concentrated upon the refraction of radio waves as well as the refractive index structure of the lower atmosphere on both synoptic and climatic scales. These are the areas of radio meteorology that are treated in this volume, with additional chapters on radio-meteorological parameters and the absorption of radio waves by the various constituents of the lower atmosphere. An effort has been made to include results obtained in other laboratories both in the United States and abroad.
\end{abstract}




\title{
Thermal-energy associative-detachment reactions of negative ions
}

\author{
Fred C. Fehsenfeld, Eldon E. Ferguson, and Arthur L. Schmeltekopf \\ ESSA Institute for Telecommunication Sciences and Aeronomy
}

Journal of Chemical Physics, 43(5): 1844-1845, 1966

\section{Significance}

This paper describes the discovery of an entirely new kind of reaction that occurs in the atmosphere, which the authors name "associative-detachment" reactions. In these reactions, a negatively charged molecule or atom reacts with a neutral species to release a "free" (unattached) electron and produce a new molecule. In the Earth's upper atmosphere, any process that releases free electrons is highly significant-electrons are the governing influence on the transmission of radio waves in the atmosphere. The new finding was therefore critical not only to telecommunications, but to national security and defense. The discovery came when researchers at the just-formed Aeronomy Laboratory were forging the very first understanding of the chemistry of the Earth's ionosphere (a region in the upper atmosphere, above the stratosphere, where charged particles exist because of high-energy radiation that the atmosphere receives at those high altitudes). It was critically important to develop a scientific understanding of the chemistry of the ionosphere, because of its influence on radio transmissions and the implications for communications and national security. This paper was a true breakthrough, because it announced that a whole new category of reactions existed that had a high impact on radio transmission in the atmosphere. The paper made impressive headway in announcing first results. Usually, a paper describes only one or a very small number of reactions - but Fehsenfeld et al. identified fifteen examples of the new types of reaction and specified the rates (speeds) at which they occur. As lead author Fred Fehsenfeld recalls, the researchers sensed that, as they discovered this new type of reaction in the laboratory, they were indeed working on a very exciting new frontier of atmospheric science. They were eager to announce their discovery, publishing it rapidly in the "letters" section of the Journal of Chemical Physics. It was a true "first" in the field of atmospheric chemistry.

\begin{abstract}
The ESSA-flowing afterglow reaction system which has been extensively used for thermalenergy positive-ion-neutral reaction studies has now been applied to negative-ion-neutral reaction studies. The following preliminary results suggest a fairly general behavior of negative ions reacting with neutrals, which has not been previously recognized. This is that many exothermic associative-detachment reactions appear to be rapid at thermal energies. These results have obvious important implications for the ion chemistry of the earth's lower ionosphere, for the chemistry of electro-negative gases in electrical discharges, and for deionization in discharge afterglows and in flames.
\end{abstract}




\title{
Thermodynamic properties of $\mathrm{He}^{3}-\mathrm{He}^{4}$ solutions with applications to the $\mathrm{He}^{3}-\mathrm{He}^{4}$ dilution refrigerator
}

\author{
Ray Radebaugh \\ NBS Cryogenics Division
}

NBS Technical Note 362, 1967

\section{Significance}

This 134-page Tech Note is noteworthy for two reasons. (1) It was the first publication to provide detailed thermodynamic properties with extensive tables and graphs of mixtures of $\mathrm{He}^{3}$ and $\mathrm{He}^{4}$ liquids for temperatures in the range from $0 \mathrm{~K}$ to $1.5 \mathrm{~K}$. (2) It gave a detailed thermodynamic analysis for the first time of the $\mathrm{He}^{3}-\mathrm{He}^{4}$ dilution refrigerator, which had been invented in 1966. A few other explanations of the dilution refrigerator had existed before, but they were difficult to follow and no longer used after the explanation from this Tech Note appeared. The dilution refrigerator has become a very important tool in low temperature physics and even today most people involved with the design of such refrigerators still refer to and use the properties given in Tech Note 362.

\begin{abstract}
The thermodynamic properties of liquid $\mathrm{He}^{3}-\mathrm{He}^{4}$ solutions between 0 and $1.5^{\circ} \mathrm{K}$ are calculated by using the weakly interacting Fermi-Dirac gas model for $\mathrm{He}^{3}$ in $\mathrm{He}^{4}$. Certain experimental data below about $0.4^{\circ} \mathrm{K}$ are used to evaluate some of the parameters in the model. The properties of both $\mathrm{He}^{3}$ in $\mathrm{He}^{4}$ and the total solution are calculated for concentrations of $\mathrm{He}^{3}$ up to 30 percent. All experimental data agree very well with the calculated results, although little data exist below $0.4^{\circ} \mathrm{K}$. The calculated properties are used to analyze the behavior of the $\mathrm{He}^{3}-\mathrm{He}^{4}$ dilution refrigerator in both the continuous and single-cycle processes. The maximum heat absorption below about $0.04^{\circ} \mathrm{K}$ is found to be $82 \mathrm{~T}^{2}$ joules per mole of $\mathrm{He}^{3}$ circulated. The effect of an imperfect heat exchanger and $\mathrm{He}^{4}$ circulation on the refrigeration capacity is discussed. An analysis of two new types of single-cycle processes is also given.
\end{abstract}




\title{
Development and operation of a specialized technical information and data center (the Cryogenic Data Center)
}

\author{
Victor J. Johnson \\ NBS Institute for Basic Standards
}

Journal of Chemical Documentation 8: 219-224, 1968

\section{Significance}

The Cryogenic Data Center was established at NBS in Boulder in 1958 to "cope with the problem of organizing the world's literature" in cryogenics and providing "reliable information and data." The field of cryogenics may have started in 1883 (with the liquefaction of air), and the interest in Boulder began with the opening of the Boulder Laboratories 50 years ago. The activities of collecting, evaluating, and disseminating information have been ongoing in Boulder, with emphasis ranging from cryogenics, to key fluids, to current efforts on essentially all chemical systems; the specific techniques used from the punch cards and magnetic tapes described in this paper to automated web-based and "expert" systems have evolved over the years. The bustling Cryogenic Data Center established some of the key features of information exchange that are at the heart of the "information and knowledge management" efforts of today.

\begin{abstract}
As is typical of many specialized areas of science and technology, cryogenics has had an explosive development in the last decade and a half. The Cryogenic Data Center was established in 1958 to cope with the problem of organizing the world's literature pertinent to the field of cryogenics and furnishing this rapidly developing industry with reliable information and data. The development of this facility over the 10-year period of its existence is discussed. Included is a description of the conversion from a manual to an automated bibliographic retrieval system, the development of cataloging and indexing techniques, the development of comprehensive awareness and literature acquisition procedures with public announcement of new literature, procedures for selecting and compiling data, and finally how the needs of the sponsors and the industry are being served. The discussion emphasizes the problems that are typical in facilities of this type and the need to consider standard or generally accepted solutions. The growing trend for a network system of technical information and data centers requires a high order of compatibility among them.
\end{abstract}




\title{
Prediction of tropospheric radio transmission loss over irregular terrain: A computer method
}

\author{
Anita G. Longley and Philip L. Rice \\ ERL Institute for Telecommunication Sciences and Aeronomy
}

ESSA Technical Report ERL 79-ITS 67, July 1968

\section{Significance}

The "Computer Method" provides the first detailed, comprehensive description of the general purpose radio propagation prediction model that subsequently became the ITS Irregular Terrain Model (ITM or Longley-Rice Model). It can be applied to a wide variety of radio engineering problems. Although the model is based in part on the concepts and methods of calculation found in Tech Note 101 (Rice et al., 1965-1967, this volume), there are numerous refinements and details of the implementation that are unique to this publication. This publication preceded the advent of the widespread availability of digital terrain elevation databases, so for a number of years the model was principally used in its area (or site-general) mode. More recently, good quality digital terrain elevation databases have become widely available, so the model has been used for radio system design and planning in its point-to-point (or site-specific) mode. Two recent examples of this use include the presumption of terrestrial broadcast service (or lack thereof) for the purposes of satellite delivery of distant network signals under the Satellite Home Viewer Improvement Act and the FCC's use of the model to develop the Table of Allotments for digital television.

\begin{abstract}
This report describes a computer method for predicting long-term median transmission loss over irregular terrain. The method is applicable for radio frequencies above $20 \mathrm{MHz}$ and may be used either with detailed terrain profiles for actual paths or with profiles that are representative of median terrain characteristics for a given area. Estimates of variability in time and with location, and a method for computing service probability, are included.
\end{abstract}




\title{
Acoustic methods for remote probing of the lower atmosphere
}

\author{
C. Gordon Little \\ ERL Wave Propagation Laboratory
}

Proceedings of the Institute of Electrical and Electronics Engineers, 57(4): 571-578, April 1969

\section{Significance}

The field of radar meteorology was born in the 1950s after researchers realized that radar systems developed in WWII were sensitive to precipitation. Research in the 1960s demonstrated the usefulness of radar for studying and monitoring precipitation events and the evolution of severe storms. However, the potential of acoustic waves for probing the lower atmosphere was relatively overlooked. This paper describes for the first time the use of acoustic methods for the remote probing of a variety of atmospheric parameters, including wind velocity, temperature and humidity structure, and associated turbulence parameters. It opened up a new vein of research that eventually led to the development of so-called echo-sounders or sodars (acoustic radars) now widely available and used routinely to monitor the lower atmosphere, both in operational and research settings.

\begin{abstract}
The potential usefulness of acoustic methods for the remote probing of the lower atmosphere is reviewed. Starting with a comparison of the effects of temperature, wind, and humidity fluctuations upon the refractive index of air to electromagnetic and acoustic waves, it is shown that the fluctuations in acoustic refractive index may be expected to be about 1000 times stronger than in the radio case. The opportunities for passive and for line-of-sight remote acoustical sensing of the troposphere offered by this relatively strong interaction are briefly discussed.

Since the scattered power is proportional to the square of the refractive index fluctuations, the scatter of acoustic waves may be expected to be roughly one million times stronger than for radio waves. Based on the theoretical work of Kallistratova (but including the effects of atmospheric absorption), the system parameters required for effective acoustic echo-sounding of the lower atmosphere are deduced. It is concluded that the acoustic sounding technique could be developed to monitor, to heights of at least 1500 meters, (1) the vertical profile of wind speed and direction, (2) the vertical profile of humidity, (3) the location and intensity of temperature inversions, (4) the three-dimensional spectrum of mechanical turbulence, and (5) the three-dimensional spectrum of temperature inhomogeneity (i.e., of optical refractive index fluctuation).

Typical time and height resolutions for the proposed acoustic echo-sounders could be of the order 10 seconds and 10 meters; the spatial wave number explored could range from about $10^{-2}$ $\mathrm{m}^{-1}$ to about $400 \mathrm{~m}^{-1}$.
\end{abstract}




\section{Papers from the 1970s}

A comparison of remote sensing of the clear atmosphere by optical, radio, and acoustic radar techniques

Vernon E. Derr and C. Gordon Little, 1970

A new method of interpolation and smooth curve fitting based on local procedures

Hiroshi Akima, 1970

Theory of isoperibol calorimetry for laser power and energy measurements

E. Dale West and Kenneth L. Churney, 1970

An alternative integral equation for propagation over irregular

terrain, 2

Randolph H. Ott, 1971

Miniature ultrasensitive superconducting magnetic gradiometer and its use in cardiography and other applications

James E. Zimmerman and N.V. Frederick, 1971

Modifying the ionosphere with intense radio waves

William F. Utlaut and Robert S. Cohen, 1971

Noise thermometry with the Josephson Effect

Robert A. Kamper and James E. Zimmerman, 1971

Thermophysical properties of oxygen from the freezing liquid line to $600 \mathrm{R}$ for pressures to 5000 Psia

Robert D. McCarty and Lloyd A. Weber, 1971

Performance of NBS cryogenic flow research facility

J.A. Brennan, Douglas B. Mann, J.W. Dean, and C.H. Kneebone, 1972

Speed of light from direct frequency and wavelength measurements of the methanestabilized laser

Kenneth M. Evenson, Joseph S. Wells, F. Russell Petersen, Bruce L. Danielson,

Gordon W. Day, Richard L. Barger, and John L. Hall, 1972

Theoretical study of the ionospheric $\mathrm{F}$ region equatorial anomaly

I. Theory

David N. Anderson, 1973

Thermal conductivity of solids at room temperature and below

Greg E. Childs, Lewis J. Ericks, and Robert L. Powell, 1973 
Generation of standard EM fields using TEM transmission cells

Myron L. Crawford, November 1974

International intercomparison of complex permittivity at $9 \mathrm{GHz}$

Howard E. Bussey, Derek Morris, and E.B. Zaltsman, 1974

Man-made radio noise, Part I: Estimates for business, residential, and rural areas

Arthur D. Spaulding and Robert T. Disney, 1974

Precipitating electron fluxes formed by a magnetic field aligned potential difference David S. Evans, 1974

Theory and application of antenna arrays

Mark T. Ma, 1974

Studies of winds in the upper troposphere with a sensitive VHF radar

John L. Green, James M. Warnock, Richard H. Winkler, and Thomas E. VanZandt, 1975

Magnetic suspension densimeter for measurements on fluids of cryogenic interest

William M. Haynes, Michael J. Hiza, and Nolan V. Frederick, 1976

Study of weld flaws in the Alaska pipeline:

1. Evaluation of girth welds from the Trans-Alaska pipeline

Richard P. Reed, M. Bud Kasen, Hassel M. Ledbetter, and Harry I. McHenry, 1976

2. A fracture mechanics evaluation of flaws in pipeline girth welds

Richard P. Reed, Harry I. McHenry, and M. Bud Kasen, 1979

3. Interim report on the significance of blunt flaws in pipeline girth welds

M. Bud Kasen and R.P. Mikesell, 1980

An analysis of three weather-related aircraft accidents

Tetsuya T. Fujita and Fernando Caracena, 1977

A network analyzer incorporating two six-port reflectometers

Cletus A. Hoer, 1977

Definitions of terms for practical superconductors:

1. Fundamental states and flux phenomena

Robert L. Powell and Alan F. Clark, 1977 
2. Critical parameters

Robert L. Powell and Alan F. Clark, 1978

3. Fabrication, stabilization, and transient losses

David T. Read, Jack W. Ekin, Robert L. Powell, and Alan F. Clark, 1979

4. Josephson phenomena

Fred R. Fickett, Steven B. Kaplan, Robert L. Powell, Ray Radebaugh, and Alan F. Clark, 1980

Effect of stress on the critical current of NbTi multifilamentary composite wire

Jack W. Ekin, Fred R. Fickett, and Alan F. Clark, 1977

Kinetics of the reaction of $\mathrm{HO}_{2}$ with $\mathrm{NO}$

Carleton J. Howard and Kenneth M. Evenson, 1977

Ocean surface currents mapped by radar

Donald E. Barrick, Michael W. Evans, and Bob L. Weber, 1977

Optimum reception in an impulsive interference environment

Part I: Coherent detection

Part II: Incoherent reception

Arthur D. Spaulding and David Middleton, 1977

69

Applications of closed-cycle cryocoolers to small superconducting devices

James E. Zimmerman and Thomas M. Flynn, 1978

Picosecond domain waveform measurements

Norris S. Nahman, 1978

Radiation-pressure cooling of bound resonant absorbers

David J. Wineland, Robert E. Drullinger, and Fred L. Walls, 1978

A hybrid of Erlang $B$ and $C$ formulas and its applications

Martin Nesenbergs, 1979

Mesoanalysis of the Big Thompson storm

Fernando Caracena, Robert A. Maddox, Charles F. Chappell, and L. Ray Hoxit, 1979

"Thru-reflect-line": An improved technique for calibrating the dual six-port automatic network analyzer

Glenn F. Engen and Cletus A. Hoer, 1979 


\title{
A comparison of remote sensing of the clear atmosphere by optical, radio, and acoustic radar techniques
}

\author{
Vernon E. Derr and C. Gordon Little \\ ERL Wave Propagation Laboratory
}

Applied Optics, 9(9): 1976-1992, September 1970

\section{Significance}

The field of tropospheric remote sensing was relatively new in the 1960s. Radio techniques were just beginning to be applied in earnest to probe and monitor severe storms. Optical methods based on laser technology invented in the late 1950s were still in their infancy. Two landmark studies by Little (1969) and McAllister (1969) had only just outlined the potential of acoustic techniques. The authors of the nominated paper noted that the complexity of emerging environmental observational needs, from weather prediction to air pollution monitoring, would require not one, but a full suite of sensors probing the same atmospheric volume to extract the information needed to understand fully the physical processes involved. The authors were the first to analyze and highlight the complementary and synergistic nature of radio, optical and acoustic radar technologies as applied to the probing of the clear lower atmosphere. Although many of the techniques they discuss are not in use today, their notion of combining sensor technologies to address critical observational needs is being realized today in the concept of test beds and integrated sounding systems.

\begin{abstract}
The considerable utility of ultrasensitive microwave radar in atmospheric studies can now be augmented by lidar and acoustic-sensing techniques. The current and potential capabilities, and limitations, of these methods of remote atmospheric probing are discussed and the techniques are compared. From this analysis the conclusions emerges that a remote sensing facility consisting of these instruments probing the same volume of the atmosphere can measure many of the meteorologically significant parameters necessary to increase our understanding of the structure and dynamics of the clear lower atmosphere, and provide the spatial and temporal density of measurements necessary for weather forecasting.
\end{abstract}




\title{
A new method of interpolation and smooth curve fitting based on local procedures
}

\author{
Hiroshi Akima \\ ERL Institute for Telecommunication Sciences
}

Journal of the Association for Computing Machinery, 17(4): 589-602, October 1970

\section{Significance}

Hiroshi Akima, a Japanese mathematician, first came to ITS as an exchange scientist in the 1960s and then was invited to return as an employee. Akima was a computer enthusiast long before computers became commonplace. This paper, published in 1970, describes a clean, neat, and very accurate computer method for drawing a smooth curve that joins a set of data points on a graph. Before this, the best way to achieve such a curve was for a trained scientist or engineer to draw it manually. The method described in the paper proved very popular in a wide range of fields, resulting in 344 citations since 1975.

\begin{abstract}
A new mathematical method is developed for interpolation from a given set of data points in a plane and for fitting a smooth curve to the points. This method is devised in such a way that the resultant curve will pass through the given points and will appear smooth and natural. It is based on a piecewise function composed of a set of polynomials, each of degree three, at most, and applicable to successive intervals of the given points. In this method, the slope of the curve is determined at each given point locally, and each polynomial representing a portion of the curve between a pair of given points is determined by the coordinates of and the slopes at the points. Comparison indicates that the curve obtained by this new method is closer to a manually drawn curve than those drawn by other mathematical methods.
\end{abstract}




\title{
Theory of isoperibol calorimetry for laser power and energy measurements
}

\author{
E. Dale West and Kenneth L. Churney \\ NBS Radio Standards Physics Division
}

Journal of Applied Physics 41: 2705-2712, 1970

\section{Significance}

This paper addressed the design of calorimeters for the measurement of laser power and energy, and provided the theoretical basis for all of the primary laser radiometry standards used by NIST today. It was identified as one of the 100 most significant NBS/NIST papers at the centennial celebration.

\begin{abstract}
Laser power and energy measurements are commonly made in calorimeters operating in a constant temperature environment. Calorimeters of this type are analyzed in terms of the first law of thermodynamics and the boundary value problem describing heat flow in the calorimeter. This theory of the measurement suggests design features of the calorimeter, sources of error to be avoided in design and operation, and tests to demonstrate experimentally the adequacy of the design. The analysis shows how time-temperature data can be used to allow for the temperature gradient on the calorimeter and the heat exchange due to transients in the temperature.
\end{abstract}




\title{
An alternative integral equation for propagation over irregular terrain, 2
}

\author{
Randolph H. Ott \\ OT Institute for Telecommunication Sciences
}

Radio Science, 6 (4): 429-435, 1971

\section{Significance}

This paper presents an improved derivation for an integral equation for propagation over irregular terrain. The integral equation was tested numerically for a spherical earth (where an exact solution was available) with excellent agreement. Then a more interesting terrain profile, a Gaussian hill, was analyzed, and the solution was compared with George Hufford's earlier (1952 - the "alternative" referred to in the title) integral equation and knife-edge diffraction. The numerical solutions agreed well and predicted the focusing of the field on the near side of the hill and the shadowing on the back side. The computer code Wagner that came out of this publication has received extensive use both inside and outside the Boulder Laboratories.

\begin{abstract}
An integral equation for calculating the attenuation of radio waves propagating over irregular terrain is rederived. The integral equation is applied to three terrain profiles, and the solutions are compared with solutions obtained by using classical methods such as the residue series and diffraction theory.
\end{abstract}




\title{
Miniature ultrasensitive superconducting magnetic gradiometer and its use in cardiography and other applications
}

\author{
James E. Zimmerman and Nolan V. Frederick \\ NBS Cryogenics Division
}

Applied Physics Letters, 19: 16-19, 1971

\section{Significance}

The Superconducting QUantum Interference Device or SQUID is a magnetic field sensor so sensitive that it is easily overwhelmed by the earth's weak magnetic field. In 1971, James Zimmerman and Nolan Frederick developed a new configuration of the SQUID magnetometer that is sensitive only to spatial variations in the magnetic field. Called a gradiometer, their device can be used to measure the extremely weak fields associated with the human heart and brain, even in the ambient field of the earth. Gradiometers based on their work are now routinely used to study noninvasively the neural activity of the brain and even to pinpoint centers of abnormal activity in the brains of epileptic patients.

\begin{abstract}
A portable magnetic gradiometer was built using an rf-biased point contact device as a sensor and a superconducting flux transformer $2.5 \mathrm{~cm}$ in diameter by $10 \mathrm{~cm}$ long to couple to the gradient of the external field. The instrument is limited by fluctuations which are probably associated with an uneven boiling of the cryogenic liquids and by environmental fluctuations in some locations. It is shown that the inherent sensitivity can be increased by an order of magnitude by increasing the bias frequency from 30 to $300 \mathrm{MHz}$. A possible use of the instrument as a magnetocardiograph in an unshielded environment was demonstrated.
\end{abstract}




\title{
Modifying the ionosphere with intense radio waves
}

\author{
William F. Utlaut \\ OT Institute for Telecommunication Sciences \\ Robert S. Cohen \\ Consultant \\ NOAA Aeronomy Laboratory
}

Science, 174: 245-254, October 15, 1971

\section{Significance}

Although controversial, the ITS experiments in "ionospheric modification" were actually quite benign. The ionosphere (the earth's upper atmosphere) is important in telecommunications because it interacts with radio waves in various, not always predictable, ways. In order to study the properties of the ionosphere, high-power transmitters known as "heaters" were constructed that could radiate vertical HF beams incident on the ionosphere. These beams would briefly, temporarily, "modify" the small section of ionosphere affected, by raising its temperature slightly (far less than the sun does every day). At the same time, a probe wave would be sent up to record what occurred in the ionosphere during the transmission. One of the first heaters was built at the Department of Commerce field site in Platteville, Colorado, and was operated during the late 1960s and 1970s. This article describes the work done at Platteville and discusses preliminary results. Although ionospheric modification is no longer performed in Colorado, these studies laid the groundwork for continuing research of this type in Alaska (HIPAS and HAARP), and elsewhere in the world.

\begin{abstract}
The ionospheric modification experiments provide an opportunity to better understand the aeronomy of the natural ionosphere and also afford the control of a naturally occurring plasma, which will make possible further progress in plasma physics. The ionospheric modification by powerful radio waves is analogous to studies of laser and microwave heating of laboratory plasmas (20). "Anomalous" reflectivity effects similar to the observed ionospheric attenuation have already been noted in plasmas modulated by microwaves, and anomalous heating may have been observed in plasmas irradiated by lasers. Contacts have now been established between the workers in these diverse areas, which span a wide range of the electromagnetic spectrum. Perhaps ionospheric modification will also be a valuable technique in radio communications.
\end{abstract}




\title{
Noise thermometry with the Josephson effect
}

\author{
Robert A. Kamper and James E. Zimmerman \\ NBS Cryogenics Division
}

Journal of Applied Physics, 42: 132-136, 1971

\section{Significance}

Only a few thermal effects are understood sufficiently well that they can be used as a absolute measure of temperature. In 1971, Robert Kamper and James Zimmerman proposed and demonstrated a new absolute thermometer based on the intrinsic electrical noise of a resistor. This noise, known as Johnson noise, depends only on the resistance, the temperature, and a fundamental constant of nature called Boltzman's constant. Using a sensitive superconducting amplifier, Kamper and Zimmerman were able to measure the Johnson noise of a resistor at temperatures near absolute zero and establish a new method of thermometry. Noise thermometry has since played an important role in establishing the temperature scale in this low-temperature regime.

\begin{abstract}
Thermal noise causes a random frequency modulation of the self-oscillation of a Josephson junction, and the temperature of the noise source can be determined by analysis of the generated signal. We show that a thermometer based on this principle would be theoretically capable of measuring temperatures in the microkelvin range, and describe a prototype thermometer which has recorded noise temperatures down to $0.075 \mathrm{~K}$.
\end{abstract}




\title{
Thermophysical properties of oxygen from the freezing liquid line to $600 \mathrm{R}$ for pressures to 5000 Psia
}

\author{
Robert D. McCarty and Lloyd A. Weber \\ NBS Cryogenics Division
}

NBS Technical Note 384, 1971

\section{Significance}

The National Bureau of Standards was well known for its properties studies - comprehensive and integrated projects of experimental measurement, data collection and evaluation, model development, and finally dissemination of standard values. After the initial work in Boulder on hydrogen, the mandate of the organization was broadened, and this early work on oxygen (continuing the trend in both cryogenics and challenging materials handling issues) represents one of the earliest efforts on property dissemination. The form of this technical note, page upon page of tabular material, represents the canonical output of the 1970s. Of course, such paper tables have been superceded by computer programs and web-based information sources, and the technical information has also been refined and updated. However, the numbers in this technical note went into the design of the important propulsion systems of the day (the oxygen tanks and technologies on NASA rockets relied on these property data), and this publication was the first in an important series of property standards.

\begin{abstract}
Tables of thermophysical properties of oxygen are presented for temperatures from the melting line to $600 \mathrm{R}$ for pressures to 5000 psia. The tables include, entropy, enthalpy, internal energy, density, volume, speed of sound, specific heat, thermal conductivity, viscosity, thermal diffusivity, Prandtl number and the dielectric constant for 79 isobars. Also included in the isobaric tables are quantities of special utility in heat transfer calculations: $(\partial \mathrm{P} / \partial \mathrm{V})_{\mathrm{T}},(\partial \mathrm{P} / \partial \mathrm{T})_{\rho}$, $\mathrm{V}(\partial \mathrm{H} / \partial \mathrm{V})_{\mathrm{P}}, \mathrm{V}(\partial \mathrm{P} / \partial \mathrm{U})_{\mathrm{V}},-\mathrm{V}(\partial \mathrm{P} / \partial \mathrm{V})_{\mathrm{T}}, 1 / \mathrm{V}(\partial \mathrm{V} / \partial \mathrm{T})_{\mathrm{P}}$

In addition to the isobaric tables, tables for the saturated vapor and liquid are given which include all of the above properties, plus the surface tension. Tables for the P-T of the freezing liquid, index of refraction and the derived Joule-Thomson inversion curve are also presented. The specific heat of the saturated liquid is given in graphical form. A temperature-entropy chart and a Mollier diagram are also included.
\end{abstract}




\title{
Performance of NBS cryogenic flow research facility
}

\author{
J.A. Brennan, Douglas B. Mann, J.W. Dean, and C.H. Kneebone \\ NBS Cryogenics Division
}

Advances in Cryogenic Engineering, Vol. 17, Plenum Press, NY, 199-205, 1972

\section{Significance}

The cryogenic flow research facility, now known as the "flow loop," has been housed in Building 3 on the Boulder campus for some 40 years. This unique facility - the only source of cryogenic flow calibrations in North America - is the connection between NIST standards and such diverse activities as measuring the amounts of cryogenic fuels in the space program and certifying the quantity of liquid nitrogen sold to hospitals. This paper provides an early performance assessment of the flow loop, and is the predecessor to the current uncertainty evaluations in flow metering. Although many components of the flow loop have been updated, this paper serves as a reference to the early protocols in cryogenic flow measurement.

\begin{abstract}
As the paper has no abstract, the introduction is given here)
The historical background and design of the Cryogenic Flow Research Facility have been given previously by Dean et al. This report describes the present status of knowledge with respect to performing cryogenic flow measurements after a year of operation of the facility. The sources of error presented here details just the contribution of the flow facility itself to the uncertainty of flow measurements. Subsequent reports will deal with the problems and uncertainties in using the facility in research measurements and in evaluation of flowmeters. The uncertainties given here are limits to the discrimination possible in these subsequent investigations.
\end{abstract}




\title{
Speed of light from direct frequency and wavelength measurements of the methane-stabilized laser
}

Kenneth M. Evenson, Joseph S. Wells, F. Russell Petersen, Bruce L. Danielson, Gordon W. Day, Richard L. Barger, and John L. Hall

NBS Quantum Electronics Division

Physical Review Letters 29: 1346-1349, 1972

\section{Significance}

This paper is one of the most important papers related to lasers, not only providing a new and 100-fold improvement in the determination of the speed of light, but leading directly to the redefinition of the meter. It was identified as one of the 100 most significant NBS/NIST papers at the centennial celebration.

\begin{abstract}
The frequency and wavelength of the methane-stabilized laser at $3.39 \mu \mathrm{m}$ were directly measured against the respective primary standards. With infrared frequency synthesis techniques, we obtain $v=88.376181627(50) \mathrm{THz}$. With frequency-controlled interferometry, we find $\lambda=$ 3.392231376 (12) $\mu \mathrm{m}$. Multiplication yields the speed of light c = $299792456.2(1.1) \mathrm{m} / \mathrm{sec}$, in agreement with and 100 times less uncertain than the previously accepted value. The main limitation is asymmetry in the krypton $6057-\AA$ line defining the meter.
\end{abstract}




\title{
Theoretical study of the ionospheric $F$ region equatorial anomaly I. Theory
}

\author{
David N. Anderson \\ NOAA/CIRES
}

Planetary and Space Science, 21: 409-419, 1973

\section{Significance}

Prior to the publication of this paper, significant observed longitudinal differences in the low latitude ionosphere could not be explained theoretically. Incorporating into a model, complex transformation techniques that realistically reproduced the Earth's geomagnetic field, the paper demonstrated that this one unifying coordinate system for both magnetic and electric fields was sufficient to account for the longitude variability in the low latitude ionosphere. The model described here theoretically solves the time dependent, coupled ion continuity and momentum equations in the equatorial $\mathrm{F}$ region ionosphere and provides ion and electron densities as a function of altitude, latitude and local time. This was the first time that a realistic geomagnetic field (represented by Euler potentials) had been included in a time dependent ionospheric model. This model has been the basis for a number of wide-ranging studies over the last three decades, on topics including: (a) ionosphere/neutral wind effects, (b) F-region ionospheric storm effects, (c) motion of depleted plasma flux tubes in the equatorial ionosphere, (d) generation of high latitude polar cap patches of ionization and, more recently, (e) data assimilation techniques as inputs to a Global Assimilation of Ionospheric Measurements (GAIM) model.

\begin{abstract}
When observed noontime values of the maximum electron density, $\operatorname{NNAX}(\mathrm{F} 2)$ in the ionospheric $\mathrm{F} 2$ region are plotted as a function of magnetic latitude, a curve is produced which has two peaks, one on either side of the dip equator at $\pm 16^{\circ}$ dip equator. This paper theoretically investigates the daily variation of this latitudinal distribution of NMAX(F2) (the so-called Appleton or equatorial anomaly) and specifically attempts to account for the longitudinal differences observed between the American and Asian sectors.

Part I outlines the theory involved in solving the time-dependent plasma continuity equation in which production, loss, and transport of ionization are taken into account, where the effects of neutral wind, ambipolar diffusion and ExB drift are included in the transport term. By describing the geomagnetic field in two equivalent ways, $\mathrm{B}=-\nabla \gamma$ and $\mathrm{B}=\nabla \alpha \times \nabla \beta$, where $\alpha, \beta$ and $\gamma$ are known magnetic scalar potentials, the spherical r, $\theta$ and $\phi$ space coordinates of the continuity equation are transformed to coordinates which define directions parallel and perpendicular to the magnetic field thus putting the equation in a form suitable for numerical integration.
\end{abstract}




\title{
Thermal conductivity of solids at room temperature and below
}

\author{
Greg E. Childs, Lewis J. Ericks, and Robert L. Powell \\ NBS Cryogenics Division
}

NBS Monograph 131, 1973

\section{Significance}

This monograph presents graphs of most of the published data at that time on the thermal conductivity of most solids of interest to scientists and engineers for temperatures below room temperature. This 608-page monograph provides very valuable data for the design of any cryogenic equipment. Such data was scattered throughout the literature before that time and difficult for most researchers to find. Because of the popularity of this monograph it soon became out of print. Such a monograph as this was needed at a time before such information could be made available electronically.

\begin{abstract}
An extensive compilation is given of the measured values of thermal conductivity for nearly all solid materials from room temperature down to 0.02 kelvin. The reviewed materials include elements, alloys and commercial metals, semiconductors, semimetals, ionic and valence crystals, minerals, molecular crystals, polymers, glasses, and disordered dielectrics. Excluded are foams, powders, earths, fibers, layers of composites, and similar other heterogeneous solids. Data for temperatures below 1 kelvin are presented separately. The tables and graphs are complete for literature references from 1900 to mid-1971. Experimental methods and physical phenomena are discussed in the text and coded in the tables for references for which curves are shown.
\end{abstract}




\title{
Generation of standard EM fields using TEM transmission cells
}

\author{
Myron L. Crawford \\ NBS Electromagnetic Fields Division
}

IEEE Transactions on Electromagnetic Compatibility, EMC-16(4): 189-195, November 1974

\section{Significance}

This paper describes the use of the transverse electromagnetic (TEM) mode in an expanded transmission line to generate a known electromagnetic (EM) field for probe calibration and the immunity testing of devices. This paper is basis for the development of TEM cells as electromagnetic compatibility (EMC) test device. TEM cells have become a standard tool at NIST and in the EMC community. Numerous standards based on TEM cells have been written covering tests for immunity, emissions, automotive components, probe calibrations, and biological effects. Many in the EMC community still refer to TEM cells as "Crawford Cells" in recognition of Crawford's role in pioneering this methodology.

\begin{abstract}
A new technique developed at the National Bureau of Standards (NBS) for establishing standard, uniform, electromagnetic (EM) fields in a shielded environment is described. The technique employs transverse electromagnetic (TEM) transmission cells that operate as $50 \Omega$ impedancematched systems. A uniform TEM field is established inside a cell at any frequency of interest below that for which higher order modes begin to propagate. Standard field strength levels from $10 \mu \mathrm{V} / \mathrm{m}$ to $500 \mathrm{~V} / \mathrm{m}$ can be established with uncertainties of less than $1.0 \mathrm{~dB}$ to $2.0 \mathrm{~dB}$ inside the NBS cells for frequencies from dc to $500 \mathrm{MHz}$. The cells are especially useful for calibrating EM radiation hazard meters, for emission and susceptibility testing of small to medium sized equipment, and for special low level calibration of very sensitive field strength meters.
\end{abstract}




\title{
International intercomparison of complex permittivity at $9 \mathrm{GHz}$
}

\author{
Howard E. Bussey \\ NBS Electromagnetics Division \\ Derek Morris \\ National Research Council of Canada \\ E.B. Zaltsman \\ Gosstandart, USSR
}

IEEE Transactions on Instrumentation and Measurements, IM-23: 235-238, 1974

\section{Significance}

This paper summarizes the measurement results of the international intercomparison between the NBS, National Research Council, Canada, and the All-Union Scientific-Research Institute of Physico-Technical and Radio-Technical Measurements (VNIIFTRI) USSR. This project provided a much needed check on the calculation of permittivity throughout the world. The materials used were easily available so it allowed other researchers to compare their results.

\begin{abstract}
Dielectric constant and loss measurements made by three government laboratories (of the USSR, Canada, and the U.S.A.) are compared. The two materials measured were glasses. The measurements utilized cavity resonators in the $H_{01 n}$ mode. The errors in dielectric constant reported by the laboratories were usually \pm 0.3 percent; actual differences between laboratories of the average corrected results were only \pm 0.05 percent. The loss tangent results disagreed when multimoding occurred; however, the errors may be as low as \pm 0.00002 or \pm 3 percent, whichever is the larger, if multimoding is avoided.
\end{abstract}




\title{
Man-made radio noise, Part I: Estimates for business, residential, and rural areas
}

\author{
Arthur D. Spaulding and Robert T. Disney \\ OT Institute for Telecommunication Sciences
}

OT Report 74-38, June 1974

\section{Significance}

This report is a description of a comprehensive effort by the Institute for Telecommunication Sciences to measure and analyze man-made radio noise in business, residential, and rural areas. The measurements were comprehensive in that they included approximately 30 locations in each of the areas and 10 frequencies separated approximately by octaves and ranging from $250 \mathrm{KHz}$ to $250 \mathrm{MHz}$. The analysis was also comprehensive in that it included the characterization of the variability of the measured parameters over frequency, widely spaced locations, and time withinthe-hour. Part II of this report (OT Report 75-63, published in May 1975), is an equally comprehensive bibliography of noise measurement data, applications, and methods. Intended to be read by a non-mathematician audience, the material in this report is still very useful today. Ultimately, results from this work were used as a world standard in the International Radio Consultative Committee (CCIR), the predecessor standards body of the International Telecommunication Union.

\footnotetext{
Abstract

The Office of Telecommunications, Institute for Telecommunication Sciences (OT/ITS), over the past several years, has accumulated a data base of man-made radio noise measurements in the frequency range from $250 \mathrm{kHz}$ through $250 \mathrm{MHz}$ taken in a number of geographical areas. This data base has been analyzed to provide estimates of the expected characteristics of man-made radio noise in business, residential, and rural areas. The parameters used are the average available power spectral density, the ratio of the rms to the average voltage of the noise envelope, and the ratio of the rms to the average logarithm of the envelope voltage. The variation of these parameters as a function of frequency, location, and time are shown and discussed. Examples of amplitude and time statistics of the received man-made radio noise process also are shown and discussed. The use of the estimates is shown (principally by references in Part II, Bibliography) in the solution of problems encountered in frequency management and telecommunication system design.
} 


\title{
Precipitating electron fluxes formed by a magnetic field aligned potential difference
}

\author{
David S. Evans \\ NOAA Space Environment Laboratory \\ Journal of Geophysical Research, 79: 2853, 1974
}

\section{Significance}

This paper, literally overnight, rendered a hitherto unacceptable explanation for aurora entirely credible. The paper did so by modeling the consequences of assuming that only the unacceptable explanation (electrons energized through an electrical potential established parallel to the geomagnetic field) was true and then demonstrating that the modeled consequences agreed in great detail with observations. To gain agreement with only a single assumption is an example of Occam's Razor principle so cherished by scientists: the theory that explains the most observations by using the fewest assumptions must be the most correct.

While before 1974 electrical potential differences along the geomagnetic field had been proposed to account for the energization of auroral electrons, the suggestion had been almost universally discarded on the basis of both theoretical arguments and features in the auroral electron distribution thought to be inconsistent with acceleration through a potential difference. This paper modeled the expected energy distribution of electrons accelerated through a potential difference, taking into account the role of backscattered and secondary electrons produced from the atmosphere. The modeled distribution both naturally accounted for features and agreed with actual observations. The paper made the idea of charged particle acceleration through magneticfield aligned potential respectable, and thus stimulated a great deal of work on the origin of such an electric field geometry. Since publication, the paper has been cited 287 times in the literature; 47 times since 1998, attesting to its long-lived impact on the field of auroral physics.

\footnotetext{
Abstract

A model is developed in which a magnetic field aligned potential difference is assumed to accelerate electrons downward into the atmosphere. It is pointed out that the upgoing backscattered electrons produced by this electron beam may process insufficient kinetic energy to overcome the hypothetical potential difference. These electrons will be reflected downward to appear as members of a precipitating electron population. A numerical model was constructed in order to describe the total precipitating electron flux in terms of a primary accelerated beam and backscatter from the atmosphere. It is pointed out that many features that appear in the model are observed in the auroral electron beam as well. An example of an auroral beam, measured by Frank and Ackerson (1971), is compared favorably with a model electron beam formed by a parallel potential.
} 


\title{
Theory and application of antenna arrays
}

\author{
Mark T. Ma \\ OT Institute for Telecommunication Sciences, and \\ University of Colorado
}

New York: John Wiley \& Sons, Inc., 1974

\section{Significance}

In addition to standard array theory for one- and two-dimensional arrays of point sources in free space, this book includes the effects of lossy ground and real antennas, making it useful in determining the effects of ground losses and reflections. It also allowed the inclusion of mutual coupling effects. Practical antenna arrays, such as Yagi-Uda antennas and log-periodic dipole arrays, were treated in detail along with numerical results. Traveling-wave antennas, such as the sloping vee, sloping rhombic, and side-terminated vertical half-rhombic antennas, were also analyzed. The numerical results and elegant mathematics combine to make this book still useful to both engineers and theoreticians.

\begin{abstract}
Chapter 1 introduces the reader to the fundamentals of linear arrays of discrete elements. It starts with the analysis for simple uniform arrays and then proceeds to arrays with tapered amplitudes, phases, and spacings. A relatively new approach, using finite Z-transforms for analyzing arrays with nonuniform amplitude excitations, is presented in detail with illustrative examples. Difference patterns produced by a monopulse array are also formulated and studied. Chapter 2 offers many recently developed techniques for synthesizing various linear arrays. It is here that power and field patterns are equally emphasized. Considerable effort is devoted to the understanding of arrays with equal sidelobes. In addition to the synthesis of array patterns, optimization of the array directivity is also thoroughly discussed. As an extension to the material presented in the first two chapters, both analysis and synthesis of two-dimensional arrays are given in Chapter 3. Greater attention is placed on ring and elliptical arrays.

In Chapter 4, currents, impedances, fields, and power gains for simple antennas such as dipoles, monopoles, and sleeve antennas above lossy ground are presented in detail. Equal attention is also given to arrays of these antennas in the form of the Yagi-Uda antenna, the curtain array, or the Wullenweber antenna. The basic principle and analytical formulation for a class of broadband antennas, namely, the log-periodic dipole array above lossy ground, are given in Chapter 5. Placing this array in alternative geometric positions (relative to the ground) to yield maximum radiations at low or high angles from the ground plane is also explored for different possible applications. In Chapter 6, a few commonly used traveling-wave antennas above lossy ground are analyzed.
\end{abstract}




\title{
Studies of winds in the upper troposphere with a sensitive VHF radar
}

\author{
John L. Green, James M. Warnock, Richard H. Winkler, and Thomas E. VanZandt \\ NOAA Aeronomy Laboratory
}

Geophysical Research Letters, 2(1): 19-21, 1975

\section{Significance}

This paper announces the development of the forerunner of the wind-profiling radar used widely today for studying winds in the troposphere (lower atmosphere) and above. By the late 1960s, researchers had noted that as radar waves propagate through the atmosphere, part of their energy is reflected back to the radar instrument, even in clear air. Researchers proposed that these echoes might be used as a measure of the wind speed in the atmosphere, because of the measurable change in the echo caused by having been reflected off a moving parcel of air (the "Doppler" effect). Some early studies made with existing large radars indicated that the approach had promise. But Aeronomy Lab scientists set out to design and build a radar specifically for wind measurements. It was located at Sunset, Colorado, in the foothills near Boulder. This paper announces their success and presents their first data gathered on lower-atmosphere winds. They compare the radar measurements with measurements made from balloon-borne instruments (the method used by meteorologists at that time). The results confirmed that the radar technique was a viable new method of obtaining wind data in the lower atmosphere.

The new technique offered a "quantum leap" in meteorological capabilities, permitting a much higher cadence of observations than balloons. Later research led to wind profilers that can operate unattended in remote locations. Aeronomy Lab partnerships with scientists in NOAA's former Wave Propagation Lab (now Environmental Technology Lab) helped to transition the wind profiling technology into operational use, and the Forecast Systems Lab developed the NOAA Profiler Network in the late 1980s and early 1990s. Wind-profiling radars are a fundamental tool in today's meteorological science and weather forecasting.

\begin{abstract}
A large $40.475 \mathrm{MHz}$ Doppler radar has been constructed near Sunset, Colorado, $16 \mathrm{~km}$ west of Boulder, in order to study winds, the troposphere, stratosphere, and mesosphere. Early results from the troposphere are presented here. It is concluded that: (1) With a sensitive VHF radar it is possible to detect echoes from throughout the upper troposphere almost all the time, under both clear and cloudy conditions; (2) Horizontal winds derived from the Doppler shifts of the echoes are consistent at heights more than $2 \mathrm{~km}$ above the local terrain with rawinsonde winds measured over $55 \mathrm{~km}$ away; (3) The reflectivity of the scatterers in the troposphere is not strongly aspect sensitive, up to an angle of $30^{\circ}$ from the zenith.
\end{abstract}




\title{
Magnetic suspension densimeter for measurements on fluids of cryogenic interest
}

\author{
William M. Haynes, Michael J. Hiza, and Nolan V. Frederick \\ NBS Institute for Basic Standards, Cryogenics Division
}

Review of Scientific Instruments 47(10): 1237-1250, 1976

\section{Significance}

The use of Archimedes' principle to measure the density of fluids certainly has a long history, but the use of magnetic suspensions to isolate the force determination from the system being studied is more recent, with much of the technology having been developed at the Boulder Laboratories. The use of large-scale liquefaction and global commerce in cryogenic natural gases (LNG) has made the density metrology for such cryogenic systems of great importance. The apparatus described in this paper was used to provide accurate, consistent, and comprehensive density data for liquefied natural gas mixtures. It could operate over a wide range of temperature and pressure with the precision and accuracy in the measurements required for anticipated technical applications. The uncertainty assessments that comprise a major part of this paper have tied commerce in LNG to fundamental NIST measurement standards.

\begin{abstract}
An apparatus incorporating a magnetic suspension technique has been developed for density measurements on liquids and liquid mixtures, particularly at saturation, at temperatures between 90 and $300 \mathrm{~K}$ and at pressures to $5 \mathrm{MPa}$ (approximately $50 \mathrm{~atm}$ ). The feasibility of adapting this method, previously used at room temperature, for low temperature use had been demonstrated in an earlier study with a density measurement on saturated liquid nitrogen near its normal boiling point. The present apparatus, which is significantly improved, and in most respects different from the earlier model, is described in detail. It includes a cryostat for continuous wide-range temperature control, a windowed equilibrium cell particularly suited for studies of liquid mixtures, and a new electronic servocircuit with a linear differential transformer for position control of the magnetic buoy. Extensive tests and density measurements have been carried out to evaluate the performance of this apparatus. Densities of saturated liquid nitrogen between $95 \mathrm{~K}$ and $120 \mathrm{~K}$ and saturated liquid methane between $105 \mathrm{~K}$ and $160 \mathrm{~K}$ are reported. The estimated standard deviation of a single density measurement is less than $0.02 \%$. The total systematic error in the measurement process from known sources is approximately $0.05 \%$. The total uncertainty of a single density measurement, which is taken as three times the standard deviation plus the systematic error, is approximately $0.1 \%$. Comprehensive comparisons of the present results with previous experimental data are presented.
\end{abstract}




\title{
Study of weld flaws in the Alaska pipeline:
}

\author{
1. Evaluation of girth welds from the Trans-Alaska pipeline \\ Richard P. Reed, M. Bud Kasen, Hassel M. Ledbetter, and Harry I. McHenry \\ NBS Fracture and Deformation Division
}

Second Report to the Office of Pipeline Safety Operations, September 29, 1976

2. A fracture mechanics evaluation of flaws in pipeline girth welds

Richard P. Reed, Harry I. McHenry, and M. Bud Kasen

NBS Fracture and Deformation Division

Welding Research Council Bulletin 245, 1979

\section{Interim report on the significance of blunt flaws in pipeline girth welds}

M. Bud Kasen and R.P. Mikesell

NBS Fracture and Deformation Division

Proceedings, International Conference on Pipeline and Energy Plant Piping, Calgary, Alberta, Canada, November 10-13, 1980

\section{Significance}

The Alaska pipeline was constructed of $1.22 \mathrm{~m}$ diameter steel pipe sections that were joined in the field by manual welding. Construction was subject to various commercial and federal codes, with relatively conservative acceptance criteria, which prescribed radiographic inspection of the girth (end-to-end) joints. The inspection included a quick evaluation during construction, and some later reexamination. As the pipeline approached completion, the reevaluation revealed flaws which may have exceeded the weld-quality standards. Some were in locations where the pipeline passed under rivers or were in other environmentally sensitive areas, where an unnecessary repair could cause more harm to the environment or the pipeline. The Office of Pipeline Safety Operations asked NBS to help evaluate the suspect welds based on the actual service requirements.

NBS used a fracture mechanics approach to determine whether the flaws shown by the radiographs would affect the fitness-for-service of the pipeline, using the actual pipeline pressures, operating temperatures, and material properties, rather than the conservative approximations built into the construction codes. In addition, NBS tested pipeline sections with flaws under simulated service conditions and was able to develop techniques to estimate flaw depths from the radiographs. This was the first use of fracture mechanics to evaluate the significance of flaws in pipeline girth welds. They showed that this material was highly resistant to brittle fracture from these blunt flaws under realistic pipeline operating conditions (and so was safe to operate), but cautioned that further work was needed to assess the long-term effects of stress corrosion. 


\title{
An analysis of three weather-related aircraft accidents
}

\author{
Tetsuya T. Fujita \\ Department of the Geophysical Sciences, University of Chicago \\ Fernando Caracena \\ NOAA Atmospheric Physics and Chemistry Laboratory
}

Bulletin of the American Meteorological Society, 58: 1164-1181, 1977

\section{Significance}

This paper is a historical landmark in both meteorology and air safety. Fujita had earlier identified what he hypothesized as wind damage footprints of severe downdrafts (later termed microbursts) in his areal storm damage studies. He showed the plausibility of downbursts as a threat to aviation in a companion paper Fujita and Beyers (1977): Spearhead echo and downbursts in the crash of an airliner. Mon. Wea. Rev., 105, 129-146. However, his hypothesis remained controversial until this paper. Working independently, Dr. Fernando Caracena had collected and analyzed data from wind towers and other pressure and temperature sensors that constituted a de facto meteorological mesonetwork surrounding the crash site of Continental flight 426 on 7 August 1975. Dr. Caracena's definitive analysis (NTSB exhibit No. 5E-1 of the Stapleton Accident, Washington, D.C., 12 pp.) led the National Transportation Safety Board to conclude that this accident was the result of windshear. Caracena brought the critical piece of evidence to Fujita's downburst hypothesis, which definitively showed that not only could cumulus convection result in downdraft-induced, damaging winds, but that such a phenomenon could also bring down a modern jetliner. This paper paved the way for many followup studies and field projects, which resulted in the construction of Terminal Doppler Radars at major airports and new pilot training programs. Consequently, since 1994, there have been no air disasters in the United States attributable to microbursts.

\begin{abstract}
Two aircraft accidents in 1975, one at John F. Kennedy International Airport in New York City on 24 June and the other at Stapleton International Airport in Denver on 7 August, were examined in detail. A third accident on 23 June 1976 at Philadelphia International Airport is being investigated. Amazingly, there was a spearhead echo just to the north of each accident site. The echoes formed from 5 to $50 \mathrm{~min}$ in advance of the accident and moved faster than other echoes in the vicinity. These echoes were photographed by National Weather Service radars, 130-205 km away. At closer ranges, however, one or more circular echoes were depicted by airborne and ground radars. These cells were only 3-5 km in diameter, but they were accompanied by downdrafts of extreme intensity, called downbursts. All accidents occurred as aircraft, either descending or climbing, lost altitude while experiencing strong wind shear inside downburst cells.
\end{abstract}




\title{
A network analyzer incorporating two six-port reflectometers
}

\author{
Cletus A. Hoer \\ NBS Electromagnetics Division \\ IEEE Transactions on Microwave Theory and Techniques, MTT-25(12): 1070-1074, \\ December 1977
}

\section{Significance}

This paper by Cletus Hoer was the first publication that described the dual six-port technique for making network analyzer measurements. The dual six-port network analyzer, used to measure broadband (2-18 GHz), complex input and output wave variables from any linear, reciprocal two-port circuit, was a significant advancement to the microwave industry in that it provided a simpler and more accurate method for measuring complex scattering parameters and power. The instrument described here provides calibration capabilities superior to commercially available four-port network analyzers, since its measurement "heads" may be connected directly to a device-under-test without connecting cables. The dual six-port network analyzer has been used as a calibration tool in many national metrology laboratories and universities. It has also been the subject of many publications both nationally and internationally since its invention in 1977 . The dual six-port continues to be a significant invention in that it provides a simple technique for building a microwave network analyzer. The technique is still viable today particularly for measurements at millimeter and higher frequencies. This instrument is still the national standard, in use in the Electromagnetics Division to calibrate circuits and devices for U.S. industry.

\begin{abstract}
This paper outlines the theory and design of a microwave network analyzer capable of measuring the network parameters of any linear reciprocal or nonreciprocal, active or passive two port. An RF signal from one source is applied at the same time to two six-port reflectometers which measure the incident and reflected waves at the ports of the two port being measured. An experimental dual six-port network analyzer for the 2 to $18 \mathrm{GHz}$ range has been completed and is described briefly. Some advantages of the proposed design over existing network analyzer designs are (1) only one source is needed, (2) no phase detectors are required, (3) no flexible cables or arms are used between the reflectometers and the two port being measured, and (4) self-calibration techniques are readily applied.
\end{abstract}




\title{
Definitions of terms for practical superconductors:
}

NBS Thermophysical Properties Division

\section{Fundamental states and flux phenomena}

Robert L. Powell and Alan F. Clark

Cryogenics 17(12): 697-701, December 1977

\section{Critical parameters}

Robert L. Powell and Alan F. Clark

Cryogenics 18(3): 137-41, March 1978

\section{Fabrication, stabilization, and transient losses}

David T. Read, Jack W. Ekin, Robert L. Powell, and Alan F. Clark

Cryogenics 19(6): 327-32, June 1979

\section{Josephson phenomena}

Fred R. Fickett, Steven B. Kaplan, Robert L. Powell, Ray Radebaugh, and Alan F. Clark Cryogenics 20: 319-25, June 1980

\section{Significance}

This sequence of four articles defined for the first time in a physically accurate and meaningful way all of the terms used in superconductivity. Practical superconductors were first developed in the late 1960s and were only beginning to find applications in the mid-1970s. The strong low temperature background and early studies of superconductivity in the Cryogenics Division enabled the authors of these works to develop the sound basis needed for the development of measurements and standards in this rapidly growing field. The complete set of definitions was adopted by the then new ASTM Committee on Superconductivity as their first standard and led to several continuing efforts to ease the applications of practical superconductors into the marketplace. These efforts continue to this day, developing measurement techniques for new products and applications of superconductivity. The standardization efforts now involve the International Standards Organization in which NIST staff still play a key role.

\begin{abstract}
The definitions of terms used in describing the phenomenology and measurement practices of practical superconductive materials are proposed. The definitions cover the subject categories of: 1, fundamental states and flux phenomena; 2, critical parameters; 3 , fabrication, stabilization, and transient losses; and 4, Josephson phenomena. It is intended that these terms will become the basis for the development of standard measurement practices and responses are invited.
\end{abstract}




\title{
Effect of stress on the critical current of NbTi multifilamentary composite wire
}

\author{
Jack W. Ekin, Fred R. Fickett and Alan F. Clark \\ NBS Thermophysical Properties Division
}

Advances in Cryogenic Engineering, Vol. 22, New York: Plenum Press, 449-452, 1977

\section{Significance}

The practical applications of superconducting wires and the first functional superconducting magnets made from those wires were having frequent failures in the early 1970s. The staff of the Cryogenics Division, having experience in low temperature research, the mechanical properties of structural materials, and the electrical properties of superconductors, concluded that there must be a mechanical/electrical interaction. Starting with a superconducting wire wrapped around a piece of broom handle inserted into the grip of a low temperature tensile test machine, they initiated one of the longest running research programs at NBS/NIST. This publication is the report of the first observation of the effect of stress on the critical current in superconductors, demonstrating that the ability of a superconductor to carry electrical current without resistance is degraded when the conductor is under stress. Subsequent programs studied both tensile and compressive stress in superconducting wires, cables, composites, and magnet structures and discovered patterns of uniform behavior and scaling laws. These now allow both understanding of the fundamental physics of the effect as well as sophisticated engineering design.

Abstract (As the paper has no abstract, a portion of the introduction is given here)

....Typically, data on the critical-current characteristics of superconducting composites are obtained on wires that are in an unstressed state. Very little is known, however, about how the critical current behaves in wires experiencing stresses of the type described above. To measure these effects, short sample critical-current tests were made under conditions simultaneously simulating the mechanical and magnetic environments of high-field superconducting magnets. The program was initiated both to form a basis for the fundamental understanding of stress effects on superconductor stability and to provide engineering data for optimizing design and construction of high-field magnets. As will be seen, the preliminary results indicate that $\mathrm{NbTi}$ multifilamentary superconducting composite wires are not characterized by a single critical current vs. field curve, but rather by a family of curves, each corresponding to a different operating stress level. 


\title{
Kinetics of the reaction of $\mathrm{HO}_{2}$ with NO
}

\author{
Carleton J. Howard \\ NOAA Aeronomy Laboratory \\ Kenneth M. Evenson \\ NBS Time and Frequency Division
}

Geophysical Research Letters, 4(10): 437-440, 1977

\section{Significance}

This paper by Howard and Evenson studied what is perhaps the "linchpin" reaction in the lower atmosphere - the reaction of hydroperoxyl radicals $\left(\mathrm{HO}_{2}\right)$ with nitric oxide $(\mathrm{NO})$. These two very reactive and naturally occurring species are present in the atmosphere in trace amounts, yet they have a disproportionately large effect on the production of ozone in the atmosphere's protective stratospheric ozone layer. Their reaction makes nitrogen dioxide $\left(\mathrm{NO}_{2}\right)$, which almost immediately breaks down in sunlight to make ozone. Thus, the reaction of $\mathrm{HO}_{2}$ with $\mathrm{NO}$ is, in essence, an ozone-making machine in the atmosphere. Before Howard and Evenson's work, the rate of the reaction had only been estimated by indirect means, and the best estimate was too slow to explain the amount of ozone that was present in the atmosphere. Howard and Evenson set out to make the first direct measurement of the reaction's rate. The experiment was tricky, because of the extreme reactivity of the $\mathrm{HO}_{2}$ with the surfaces of the experimental apparatus. They found a value about 10 times faster than the earlier estimates. This finding has withstood the test of time, and remains the recommended value for the rate of the reaction. This work enabled atmospheric scientists to reconcile models of atmospheric chemistry with real-world measurements, thus providing a more accurate rendering of ozone chemistry to underpin decisions on the ozone layer, as well as "smog" chemistry. For example, the new understanding implied that industrially produced chlorofluorocarbons (CFCs) were even more important at depleting the ozone layer than previously thought. That insight served as input to the international decisions made later to ban the production of CFCs.

\begin{abstract}
The gas phase reaction of $\mathrm{HO}_{2}$ radicals with $\mathrm{NO}$ was studied using a discharge-flow system and laser-magnetic resonance detection. Concentrations of $\mathrm{HO}_{2}$ down to $2 \times 10^{9}$ molecules $/ \mathrm{cm}^{3}$ were detectable, thus allowing the reaction to be studied under pseudo-first-order kinetic conditions. The rate constant was measured using three independent kinetic schemes, one of which involved the detection of the reaction products $\mathrm{OH}$ and $\mathrm{NO}_{2}$. The three schemes gave results in excellent agreement. The rate constant, $\mathrm{k}_{1}$, for the reaction $\mathrm{HO}_{2}+\mathrm{NO} \rightarrow \mathrm{OH}+\mathrm{NO}_{2}$ is $(8.1 \pm 1.5) 510^{-12}$

$\mathrm{cm}^{3} /$ molecule $\cdot \mathrm{s}$ at $296 \mathrm{~K}$ and a pressure of 140 to $220 \mathrm{~Pa}$ (1.1 to $1.7 \mathrm{torr}$ ). The measurements also indicate that the rate constants for two other exothermic channels of the reaction are less than $2 \%$ of $\mathrm{k}_{1}$.
\end{abstract}




\title{
Ocean surface currents mapped by radar
}

\author{
Donald E. Barrick, Michael W. Evans, and Bob L. Weber \\ NOAA Wave Propagation Laboratory
}

Science, 10(4313): 138-144, October 14, 1977

\section{Significance}

An accurate knowledge of surface currents is critical for the sound management of our coastal waters. It aids emergency managers by helping them to predict the drift of surface pollutants; it is of interest to ocean biologists because it is an important factor in determining the distribution of near-surface zooplankton and phytoplankton, which are critical components of the ocean food chain; and it benefits the fisheries industry by providing a better insight into the drift of many types of fish eggs. Surface currents also transport water with anomalous temperature differences that can impact regional weather patterns. Prior to the 1970s, the measurement of surface currents was accomplished via moored current meters, or by tracking floating objects or material suspended in the flow. These methods were often expensive and time intensive, and did not always yield current maps with the required spatial and temporal resolution.

The nominated paper describes the first successful effort to remotely map surface currents over a large area (out to $70 \mathrm{~km}$ from shore) using a relatively inexpensive and compact shore-based radar system that offered continuous, unattended operation. It represents a much larger body of work carried out in ETL over a period of a decade or more, and is preceded by a number of groundbreaking theoretical studies describing the interaction of electromagnetic waves and the sea surface, and succeeded by a number of investigations that extended this and related technologies to include wider-region coverage offered by satellite and skywave (over-the horizon systems that employ the scatter of electromagnetic waves from the ionosphere) systems. The system described in this paper was eventually commercialized and is now used worldwide to address a variety of current mapping needs.

\begin{abstract}
Mobile coastal units can map variable surface currents in real time to $70 \mathrm{~km}$, using ocean wave scatter.
\end{abstract}




\title{
Optimum reception in an impulsive interference environment Part I: Coherent detection Part II: Incoherent reception
}

\author{
Arthur D. Spaulding \\ OT Institute for Telecommunication Sciences \\ David Middleton \\ Contractor
}

IEEE Transactions on Communications, COM-25 (9): 910-923 and 924-934, September 1977

\section{Significance}

This pair of articles provides a comprehensive review of Don Spaulding's work in signal detection in a non-Gaussian noise environment. The papers make use of a physical-statistical interference model to analyze the performance of a large class of digital systems. This model and the analysis techniques developed by the authors have found significant use for both system analysis and the physical design of receiving systems.

\begin{abstract}
Part I
Because communications systems are seldom significantly interfered with by classical white Gaussian noise, it is necessary to consider other, appropriate (and tractable) interference models, if realistic estimates of system performance are to be obtained for the general spectral-use environment. For this purpose, Middleton's recently developed canonical statistical-physical model of "impulsive" interference is applied to real-world communication channels. The principal features of this model are first summarized, including the statistical relations required for the solution of signal detection problems. [Excellent agreement of these model statistics with correspondingly measured statistics is also noted.]

The model for narrow-band impulsive interference (Class A noise, a subset of the overall model) is next specifically applied to an important class of coherent signal detection problems. Algorithms for error probabilities in optimum detection are then obtained, along with performance bounds, for the same error probabilities. Since it is known that in order to gain significant improvement over current receivers, the number of (essentially) independent samples of the received interference waveform must be enlarged (i.e., large "processing gains"), the performance results here are given parametrically in the number of samples, or equivalently, in the time-bandwidth product. Performance of current suboptimum receivers is then obtained and compared to the optimum performance. It is shown that very substantial savings in signal power and/or spectrum space can usually be achieved by using the indicated optimal algorithms.
\end{abstract}

Since physical realization of the completely optimum detection algorithms cannot, in general, be economically realized, the somewhat more conservative, corresponding locally optimum Bayes 
detection (LOBD) receivers are derived. In general, these LOBD structures require adaptive, highly non-linear filters, preceding the conventional correlation detector elements characteristic of optimum receivers for Gaussian interference. Performance for these non-linear, optimum threshold systems is then determined, specifically in Part I for coherent reception.

\begin{abstract}
Part II
In Part I, the relevant statistical properties of the recently developed statistical-physical model of generalized impulsive interference have been briefly reviewed (for sub Class A noise) and then applied specifically to optimum coherent detection. It is shown that by using optimum and (locally optimum) detection algorithms (canonically and explicitly derived), substantial savings in signal power and/or spectrum space can be achieved for operation in these highly nonGaussian interference environments. This paper (Part II) extends the preceding analysis to cover various important cases of incoherent reception. The same general model for narrow-band (Class A) impulsive interference and interference examples used in Part I are again employed here. In addition to providing both canonical LOBD structures and expressions for performance, this permits explicit quantitative comparisons between coherent and incoherent reception for common classes of specific digital signal waveforms.
\end{abstract}




\title{
Applications of closed-cycle cryocoolers to small superconducting devices
}

James E. Zimmerman and Thomas M. Flynn, editors

NBS Electromagnetic Technology Division

NBS Special Publication 508, 1978

\section{Significance}

This NBS Special Publication is the proceedings of a conference organized by Jim Zimmerman and held at NBS on October 3-4, 1977. This conference started what has become the International Cryocooler Conference (ICC) that is held every two years. The $13^{\text {th }}$ ICC was held at the end of March, 2004. The initial NBS conference reported in this special publication is now referred to as the 0th International Cryocooler conference rather than the 1st because of a mixup in naming them at a later date. What is known as the 1st was also organized by Jim Zimmerman and held at NBS in 1980. The ICC now draws about 250 attendees and over 100 papers at each conference. The proceedings are about 1000 pages each and have been published by Plenum/Kluwer Press since the 8th ICC. The ICC and its proceedings are the major source of published papers related to cryocoolers.

\begin{abstract}
This document contains the proceedings of a meeting of specialists in small superconducting devices and in small cryogenic refrigerators. Industry, Government, and academia were represented at the meeting held at the National Bureau of Standards (NBS) on October 3 and 4, 1977. The purpose of the meeting was to define the refrigerator requirements for small superconducting devices and to determine if small cryogenic refrigerators that are produced in relatively large quantities can be adapted or developed to replace liquid helium as the cooling medium for the superconducting devices. Because the focus was on small superconducting devices, the discussion was primarily limited to refrigerators with capacities from zero up to a watt or two in the temperature range of 2 to $20 \mathrm{~K}$. The meeting was jointly sponsored by the Office of Naval Research (ONR) and NBS.
\end{abstract}




\title{
Picosecond domain waveform measurements
}

\author{
Norris S. Nahman \\ NBS Electromagnetic Technology Division
}

Proceedings of Institute of Electrical and Electronics Engineers, Special Issue on

Electromagnetic Measurements Science, Invited Paper, 66(5): 441-454, April 1978

\section{Significance}

This seminal paper recognized and emphasized the importance of bringing together electrical and optical picosecond domain pulse measurement technologies into a unified technology. The technologies described in the paper ranged from DC to terahertz electrical techniques, including superconductive devices and Debye polar molecular pulse-forming filters, optical pulse generation and sampling of electrical pulse measurements, electrical and optical auto- and crosscorrelation methods, and computer controlled measurement and signal processing of picosecond domain electrical and optical pulse waveforms. The paper in its entirety was translated and published in Japanese by McGraw-Hill in Nikkei Electronics with the banner admonition, "Every electronics engineer in Japan should read this paper" (Nikkei Electronics, April 1979, Tokyo, Japan).

\begin{abstract}
A review of the state-of-the-art of picosecond time-domain measurements is presented which draws together techniques from the electrical and optical regions of the electromagnetic spectrum. Measurement methods are listed in categories, which exhibit the commonality between electrical and optical methods. State-of-the-art values for temporal resolution are presented with reference citations to specific methods and related technical topics.
\end{abstract}




\title{
Radiation-pressure cooling of bound resonant absorbers
}

\author{
David J. Wineland, Robert E. Drullinger, and Fred L. Walls \\ NBS Time and Frequency Division
}

Physical Review Letters, 40: 1639-1642, 1978

\section{Significance}

This paper described the first experimental observation of laser-cooling particles (in this case, ions) to low temperatures. It opened up a new field of using light to manipulate the translational states of atomic particles. Today, laser-cooling is used in atomic clocks and frequency standards. It is also one of the first steps in cooling atomic gases to quantum degeneracy where BoseEinstein Condensation has been observed. This work underpins the current U.S. standard for time and frequency (NIST-F1 laser-cooled atomic fountain clock), led to two Nobel prizes, and opened up a large vigorous global research effort spanning such topics as quantum computing, atom lasers, and new states of matter.

NIST Fellow Dave Wineland was one of the originators in 1975 of the concepts of using lasers to cool atoms or ions to very near absolute zero. Wineland joined NBS in 1975 and his group soon turned those concepts into reality, using laser to cool magnesium ions to about 40 Kelvin (40 degrees above absolute zero), the first demonstration of laser cooling of any species (simultaneously and independently of a demonstration by Peter Toschek and Hans Dehmelt). Wineland's group's work led directly to work by several other groups throughout the world on laser cooling and trapping of neutral atoms. Wineland's group and other NIST researchers have been world leaders in this field for more than 20 years, as demonstrated in part by the 1997 Nobel Physics Prize to NIST's Bill Phillips (along with Steve Chu and Claude Cohen-Tannoudji) for development of neutral atom laser cooling, and the 2001 Nobel Physics Prize to NIST's Eric Cornell (along with Carl Weiman and Wolfgang Ketterle) for using laser cooling to create the Bose-Einstein Condensate, a new form of ultra-cold matter. Wineland's work has led NIST researchers to current cutting-edge research on quantum computing, atomic clocks, atom lasers, fundamental research on the properties of quantum mechanics, and a host of other advances. This paper is one of the top 50 papers in physics for the 20th century.

\begin{abstract}
We report the first observation of radiation pressure cooling on a system of resonant absorbers which are elastically bound to a laboratory fixed apparatus. $\mathrm{Mg}$ II ions confined in a Penning electromagnetic trap are cooled to $<40 \mathrm{~K}$ by irradiating them with the $8-\mu \mathrm{W}$ output of a frequency doubled, single-mode dye laser tuned to the low-frequency side of the Doppler profile on the ${ }^{2} \mathrm{~S}_{1 / 2} \leftrightarrow{ }^{2} \mathrm{P}_{3 / 2}\left(\mathrm{M}_{\mathrm{J}}=+1 / 2 \leftrightarrow \mathrm{M}_{\mathrm{J}}=+3 / 2\right.$ or $\left.\mathrm{M}_{\mathrm{J}}=-1 / 2 \leftrightarrow \mathrm{M}_{\mathrm{J}}=-3 / 2\right)$ transitions. Cooling to approximately $10^{-3} \mathrm{~K}$ should be possible.
\end{abstract}




\title{
A hybrid of Erlang $B$ and $C$ formulas and its applications
}

\author{
Martin Nesenbergs \\ NTIA Institute for Telecommunication Sciences
}

IEEE Transactions on Communications, COM-27 (1): 59-68, January 1979

\section{Significance}

This paper integrates and extends the classical Erlang B ("blocked calls lost") and Erlang C ("blocked calls delayed") formulas for calculating grade of service in switched telecommunication networks by introducing a new parameter $(\theta)$ that describes customer behavior when a customer request cannot immediately be served due to network congestion. The paper is important because it defines a practical means of dimensioning and operating networks that support different types of traffic (e.g., telephone calls and store-and-forward data) and must meet grade-of-service objectives under conditions not addressed by the classical formulas (e.g., variable customer responses to blocked service requests, alternate routing, repeated call attempts, traffic control). These real-world conditions will need to be addressed in emerging multi-service networks supporting assured quality levels (e.g., packet-based NGNs).

\begin{abstract}
This paper attempts to broaden the usefulness of existing Erlang grade of service formulas, especially when faced with the evolving extensive changes in telephone networks. The old Markov traffic arrival and Markov call duration (or service) models are combined with what may be called more realistic user behavior and network scenarios. For the specified service system models, a generalized merger of ideal loss and ideal delay steady-state representations is developed. It is shown that a particular weighted inverse combination of Erlang $B$ and $C$ formulas, herein called the hybrid, is useful for grade of service estimates under several conditions. In addition to the usual offered load and the number of servers, the hybrid involves a relative Erlang $B$ versus $C$ weighting parameter $\theta$. This parameter is non-negative and depends on system applications, as illustrated in a number of examples given. When less than or equal to unity, such as for the popular Poisson model, $\theta$ has a simple meaning. It is then the conditional probability that a typical customer elects to wait, or a service request is entered into a queue, given that all servers are busy.
\end{abstract}




\title{
Mesoanalysis of the Big Thompson storm
}

\author{
Fernando Caracena, Robert A. Maddox, and Charles F. Chappell \\ NOAA Atmospheric Physics and Chemistry Laboratory \\ L. Ray Hoxit \\ Federal Aviation Administration
}

Monthly Weather Review, 107, 1-17, 1979

\section{Significance}

The study of the Big Thompson flash flood presented in this paper became a landmark because it awakened the meteorological community to the importance of forecasting flash floods. It resulted in other new initiatives related to flash floods that have since matured into programs. Several components of this future work were accomplished by members of the Mesometeorological Group, who were awarded a NOAA Unit Citation for their initial work, which paved the way for many other publications on this subject.

\begin{abstract}
Mesoscale analyses and descriptions of meteorological conditions that produced the devastating flash flood in the Big Thompson Canyon on 31 July 1976 are presented. The storm developed when strong low-level easterly winds to the rear of a polar front pushed a moist, conditionally unstable air mass upslope into the Front Range of the Rocky Mountains. The main thrust of the moisture flux focused initially into the Big Thompson area. Orographic uplift released the convective instability, and light south-southeasterly winds at steering levels allowed the storm complex to remain nearly stationary over the foothills. Heavy rains fell within the storm along a narrow corridor only $5 \mathrm{~km}$ wide oriented north-northeast by south-southwest. Minimal entrainment of relatively moist air at middle and upper levels, very low cloud bases and a slightly tilted updraft structure contributed to a high precipitation efficiency. A deep, warm layer of convective cloud fostered precipitation growth through warm cloud processes. The greatest concentrations of precipitation size particles remained at low elevations and as result of poor vertical beam resolution returned anomalously weak radar echoes to a WSR-57 located $110 \mathrm{n} \mathrm{mi}$ away.
\end{abstract}




\title{
"Thru-reflect-line": An improved technique for calibrating the dual six-port automatic network analyzer
}

\author{
Glenn F. Engen and Cletus A. Hoer \\ NBS Electromagnetic Technology Division
}

IEEE Transactions on Microwave Theory and Techniques, MTT-27(12): 987-993, December 1979

\section{Significance}

The work in this paper formalizes the use of transmission lines as impedance standards. It was a major breakthrough in that it led to a calibration scheme which did not require the use of a known short or other calibration "circuits." This laid the foundation for the use of six-port systems developed at NIST over the past 20 years. These systems cover the frequency range from $10 \mathrm{MHz}$ to $100 \mathrm{GHz}$ and are still used for NIST calibration services of thermistor detectors and $n$-port devices. This work has been extensively referenced in the literature as the methods developed in that paper remain the most accurate for calibration vector network analyzers. The myriad of calibration schemes are ultimately compared to the TRL method developed by Hoer and Engen.

\begin{abstract}
In an earlier paper, the use of a "thru-short-delay" (TSD) technique for calibrating the dual sixport automatic network analyzer was described. Another scheme required only a length of precision transmission line and a "calibration circuit." The better features of these two somewhat different approaches have now been combined and the requirement for either a known short, or a "calibration circuit" eliminated. This paper will develop the theory for this new procedure.
\end{abstract}




\section{Papers from the 1980s}

Analytical and numerical techniques for analyzing an electrically short dipole with a nonlinear load

Motohisa Kanda, 1980

Ground wave attenuation function for a spherical earth with arbitrary surface impedance

David A. Hill and James R. Wait, 1980

Interactive FORTRAN IV computer programs for the thermodynamic and transport properties of selected cryogens (fluids pack)

Robert D. McCarty, 1980

Fracture and strength properties of selected austenitic stainless steels at cryogenic temperatures David T. Read and Richard P. Reed, 1981

Plane-wave scattering-matrix theory of antennas and antenna-antenna interactions David M. Kerns, June 1981

Prediction of transport properties, 1. Viscosity of fluids and mixtures

James F. Ely and Howard J.M. Hanley, 1981

Selected properties of hydrogen (engineering design data)

Robert D. McCarty, Jesse Hord, and Hans M. Roder, 1981

A guide to the use of the ITS irregular terrain model in the area prediction mode

George A. Hufford, Anita G. Longley, and William A. Kissick, 1982

An attenuation function for multiple knife-edge diffraction

Lewis E. Vogler, 1982

Critical current measurements: A compendium of experimental results

Loren F. Goodrich and Fred R. Fickett, 1982

Optical fiber characterization, Volumes 1 and 2

George E. Chamberlain, Bruce L. Danielson, Gordon W. Day, Douglas L. Franzen,

Robert L. Gallawa, Ernest M. Kim, and Matt Young, 1982

Design and error analysis for the WR10 thermal noise standard

William C. Daywitt, December 1983

Direct frequency measurement of the I2-stabilized He-Ne 473-THz (633 nm) laser Donald A. Jennings, Clifford R. Pollock, F. Russell Petersen, Robert E. Drullinger, Kenneth M. Evenson, Joseph S. Wells, John L. Hall, and Howard P. Layer, 1983 
Large-scale meteorological conditions associated with midlatitude, mesoscale convective complexes

Robert A. Maddox, 1983

Liquefied natural gas densities: Summary of research program at the National Bureau of Standards

William M. Haynes, Robert D. McCarty, and Michael J. Hiza, 1983

Materials at low temperatures

Richard P. Reed and Alan F. Clark, 1983

The Colorado wind-profiling network

Richard G. Strauch, David A. Merritt, Kenneth P. Moran, Kenneth B. Earnshaw, and Douglas van de Kamp, 1984

A practical Josephson voltage standard at $1 \mathrm{~V}$

Clark A. Hamilton, Richard L. Kautz, and Frances L. Lloyd, 1985

Electron-tunneling experiments using $\mathrm{Nb}-\mathrm{Sn}$ "break" junctions

John Moreland and Jack W. Ekin, 1985

A comparison of three types of pulse tube refrigerators: New methods for reaching $60 \mathrm{~K}$ Ray Radebaugh, James Zimmerman, David R. Smith, and Beverly Louie, 1986

On the depletion of Antarctic ozone

Susan Solomon, Rolando R. Garcia, F. Sherwood Rowland, and Donald J. Wuebbles, 1986

Reducing the risk of refinery explosions:

1. Examination of a pressure vessel that ruptured at the Chicago refinery of the Union

Oil Company on July 23, 1984

Harry I. McHenry, T. Robert Shives, David T. Read, J. David McColskey,

Charles H. Brady, and Patrick T. Purtscher, 1986

2. Observations of hydrogen damage in a failed pressure vessel

Harry I. McHenry, Patrick T. Purtscher, and T. Robert Shives, 1987

3. Failure analysis of an amine-absorber pressure vessel

Harry I. McHenry, David T. Read, and T. Robert Shives, 1987

Models and observations of the impact of natural hydrocarbons on rural ozone

Michael Trainer, Eric J. Williams, David D. Parrish, Martin P. Buhr, Eugene J. Allwine, Halvor H. Westberg, Fred C. Fehsenfeld, and Shaw C. Liu, 1987 
Second viscosity and thermal conductivity virial coefficients of gases:

Extension to low reduced temperature

James C. Rainwater and Daniel G. Friend, 1987

Tunneling spectroscopy of a La-Sr-Cu-O break junction: Evidence for strong coupling superconductivity

John M. Moreland, Alan F. Clark, Loren F. Goodrich, Harry C. Ku,

and R.N. Shelton, 1987

Error analysis techniques for planar near-field measurements

Allen C. Newell, 1988

Josephson-junction model of critical current in granular $\mathrm{Y}_{1} \mathrm{Ba}_{2} \mathrm{Cu}_{3} \mathrm{O}_{7-\delta}$ superconductors

Robert L. Peterson and Jack W. Ekin, 1988

Handbook of basic tables for chemical analysis

Thomas J. Bruno and Paris D.N. Svoronos, 1989

MPM-An atmospheric millimeter-wave propagation model

Hans J. Liebe, 1989

Performance standards for packet-switched services

Neal B. Seitz, 1989

Stainless steel weld metal: Prediction of ferrite content

Chris N. McCowan, Thomas A. Siewert, and D.L. Olson, 1989

The accuracy of RASS temperature measurements

Peter T. May, Kenneth P. Moran, and Richard G. Strauch, 1989

Automated Computer Time Service:

1. The NIST Automated Computer Time Service

Judah Levine, Marc Weiss, Dickie D. Davis, David W. Allan, and D.B. Sullivan, 1989116

2. Time synchronization using the Internet

Judah Levine, 1998 


\title{
Analytical and numerical techniques for analyzing an electrically short dipole with a nonlinear load
}

\author{
Motohisa Kanda \\ NBS Electromagnetic Fields Division
}

IEEE Transactions on Antennas and Propagation, AP-28(1): 71-78, January 1980

\section{Significance}

This paper develops the principles for dipole probes used to measure electric fields. This basic design led to the successful development of a wide variety of NIST field probes as well as being adopted by industry as the basis for commercial probes. These short dipole probes form the backbone for many antenna calibrations, electromagnetic compatibility test site qualification, radiation hazard monitoring, and bioelectromagnetic effects characterization. Subsequent papers on field probe development and application often begin with a reference to this fundamental paper.

\begin{abstract}
An electrically short dipole with a nonlinear dipole load is analyzed theoretically using both analytical and numerical techniques. The analytical solution is given in terms of the Anger function of imaginary order and imaginary argument and is derived from the nonlinear differential equation for the Thévenin's equivalent circuit of a dipole with a diode. The numerical technique is to solve the nodal equation using a time-stepping finite difference equation method. The nonlinear resistance of the diode is treated using the Newton-Raphson iteration technique. A comparison between the analytical and numerical solutions is given.
\end{abstract}




\title{
Ground wave attenuation function for a spherical earth with arbitrary surface impedance
}

\author{
David A. Hill \\ NTIA Institute for Telecommunication Sciences \\ James R. Wait \\ NOAA Environmental Research Laboratory \\ Radio Science, 15(3): 637-643, May-June 1980
}

\section{Significance}

For layered earth models with arbitrary conductivity, permittivity, and permeability, the surface impedance can take on a large range of complex values. This paper was the first to treat groundwave propagation over a spherical earth for arbitrary surface impedance and path length. This required the use of several methods of calculation (residue series, power series, and small curvature approximation) in order to write a completely general computer code. Various propagation effects (such as the trapped surface wave for an inductive surface impedance) are illustrated numerically for a vertical electric dipole source for frequencies from 1 to $30 \mathrm{MHz}$. The computer code was requested by many users outside the Boulder Laboratories for about 10 years after publication of the paper.

\begin{abstract}
Previous methods developed for computation of the attenuation function for a homogeneous spherical earth are generalized for an arbitrary surface impedance. The power series and smallcurvature formula developed for short distances can be used for such surfaces without modification. Care must be taken, however, to include properly the trapped surface wave that may arise in the formulation. In general, it is found that the inductive type of boundary enhances the total field but, if the resistive part is small, rather strong interference takes place between the trapped surface wave and the usual ground wave. These effects are illustrated for a surface-based vertical electric dipole for frequencies from 1 to $30 \mathrm{MHz}$.
\end{abstract}




\title{
Interactive FORTRAN IV computer programs for the thermodynamic and transport properties of selected cryogens (fluids pack)
}

\author{
Robert D. McCarty \\ NBS Thermophysical Properties Division
}

NBS Technical Note 1025, 1980

\section{Significance}

The dissemination of standardized thermophysical property information from NBS evolved from reams of tables and hand-drawn curves to computer programs - on tapes, personal computers, or through the World Wide Web. This Tech Note from 1980 describes a very early version of a computer program used to calculate the properties of several fluids at arbitrary values of temperature and pressure. The work actually provides a listing of the source code used in the computer program and provides source references and even uncertainty estimates for the calculated values. This "Fluids Pack" computer program is the forerunner of several computerized standard reference databases. The program was adopted as a standard calculational tool within NASA, and thus has become part of the space program, an important part of our explorations from the moon to the stars.

\begin{abstract}
The thermodynamic and transport properties of selected cryogens have been programmed into a series of computer routines. Input variables are any two of $\mathrm{P}, \rho$ or $\mathrm{T}$ in the single phase regions and either $\mathrm{P}$ or $\mathrm{T}$ for the saturated liquid or vapor state. The output is pressure, density, temperature, entropy, enthalpy for all of the fluids and in most cases specific heat capacity and speed of sound. Viscosity and thermal conductivity are also given for most of the fluids. The programs are designed for access by remote terminal; however, they have been written in a modular form to allow the user to select either specific fluids or specific properties for particular needs.

The program includes properties for hydrogen, helium, neon, nitrogen, oxygen, argon, and methane. The programs include properties for gaseous and liquid states usually from the triple point to some upper limit of pressure and temperature which varies from fluid to fluid. Computer listings of the FORTRAN IV codings are presented.
\end{abstract}




\title{
Fracture and strength properties of selected austenitic stainless steels at cryogenic temperatures
}

\author{
David T. Read and Richard P. Reed \\ NBS Fracture and Deformation Division
}

Cryogenics, 415-417, July 1981

\section{Significance}

This paper is one of many on mechanical properties of materials at low temperatures that were produced by NIST Boulder. This particular paper established the quantitative trade-off between strength and toughness for these materials. This relationship was used in the design and selection of weld processes.

Austenitic stainless steels have an excellent combination of mechanical and physical properties for load-bearing structures of large superconducting magnets for plasma containment in magnetic fusion experiments. To assess their relative suitability, fracture toughness, fatigue crack growth, and tensile properties data for five austenitic steels at 295, 76, and $4 \mathrm{~K}$ were obtained. The steels were AISI 304, 316, 304LN, and 316LN, and an Fe-21Cr-12Ni-5Mn alloy with a higher nitrogen content than the other four grades. The two principal findings were the systematic variation of yield strength with nitrogen content and a systematic inverse correlation between fracture toughness and yield strength. Data from previous studies confirmed the trends of the present data.

\footnotetext{
Abstract

Nitrogen-strengthened austenitic stainless steels have attracted interest as cryogenic structural materials because of their superior strength but data on their toughness at cryogenic temperatures are scarce. As cryogenic structures become larger and magnetic fields more intense, materials with higher strengths than the widely used alloy AISI 304 are necessary. In fact, nitrogenstrengthened AISI 304LN and 316LN have been selected for, respectively, the magnet cases for the Magnetic Fusion Test Facility at Lawrence Livermore Laboratory and a toroidal field coil being built for the Large Coil Project. Clearly, development of a data base on the structural properties of nitrogen-strengthened austenitic stainless steels is needed to support materials selection and design for the large cryogenic magnet structures of the near future.
} 


\title{
Plane-wave scattering-matrix theory of antennas and antenna-antenna interactions
}

\author{
David M. Kerns \\ NBS Electromagnetic Fields Division
}

NBS Monograph 162, June 1981

\section{Significance}

This monograph is David Kerns' final compilation on the subject of near-field antenna measurements and was published in 1981 shortly before he retired. It remains the best and most exhaustive treatment of planar near-field scanning theory. Under the leadership of Kerns and other pioneers at NBS, near-field techniques have become the preferred methods for characterizing the radiating properties of microwave antennas. In addition to the well-known NIST antenna group, several universities maintain active near-field measurement research programs. Half a dozen companies market near-field scanning equipment and well over 200 laboratories, worldwide, routinely perform near-field measurements. The IEEE Antennas and Propagation Society has published a special issue (IEEE Transactions on Antennas and Propagation, June 1988) and regularly features sessions on near-field techniques at its conferences.

\begin{abstract}
This monograph is distinguished by the use of plane-wave spectra for the representation of fields in space and by the consideration of antenna-antenna (and antenna-scatterer) interactions at arbitrary separation distances. The plane-wave representation is eminently suitable for this purpose as well as for the expression of conventional asymptotic quantities of antenna theory, such as power gain, effective area, and polarization. The primary objective of the monograph is to facilitate the critical acceptance and proper application of antenna scattering-matrix (PWSM) theory of antennas and antenna-antenna interactions. A secondary objective is to present some recent and some new theoretical results based on this theory. The three chapters of this monograph provide (a) an introduction to the basic theory and practice of microwave network analysis (which form an inescapable part of microwave antenna measurement expertise); (b) a thorough formulation of the PWSM theory of antennas and antenna-antenna interactions, including analytical techniques for derived measurement methods; and (c) recent and new theoretical results and analytical examples. Topics in (c) include: a convenient and attractive reformulation of the PWSM theory; some theory of minimum scattering antennas; theory of classes of completely solvable antenna-antenna interaction problems; and convergent, asymptotic expansions of transmission- and reflection-integrals in reciprocal power of $r_{0}$, where $r_{0}$ is the magnitude of the relative displacement vector $\boldsymbol{r}_{0}$ and the direction of $\boldsymbol{r}_{0}$ is a parameter.
\end{abstract}




\title{
Prediction of transport properties, 1. Viscosity of fluids and mixtures
}

James F. Ely and Howard J.M. Hanley

NBS Thermophysical Properties Division

Industrial and Engineering Chemistry Fundamentals, 20: 323-332, 1981

\section{Significance}

The theoretical model described in this paper forms the basis for the most accurate and broad ranging methods used to describe and predict the viscosity surfaces for a variety of pure fluids and mixtures. The work presents an extended corresponding-states principle that allows a calculation of viscosity even when very little information on the fluid is available. The model has been extended since this early version, but is still being used in the NIST standard reference databases for such diverse systems as natural gases, refrigeration fluids, and reservoir fluids.

\begin{abstract}
A model for the prediction of the viscosity of nonpolar fluid mixtures over the entire range of PVT states is presented. The model is an extension of an earlier version (Hanley, 1976) to molecular weights which roughly correspond to that of C20. The proposed model is based on an extended corresponding states principle and requires only critical constants and Pitzer's acentric factor for each component as input. Extensive comparisons with experimental data for pure fluids and binary mixtures are presented. The average deviation between experiment and prediction is $8 \%$ for pure species and $7 \%$ for mixtures.
\end{abstract}




\title{
Selected properties of hydrogen (engineering design data)
}

\author{
Robert D. McCarty, Jesse Hord, and Hans M. Roder \\ NBS Thermophysical Properties Division
}

NBS Monograph 168, 1981

\section{Significance}

A 20-year effort at the Boulder Laboratories on the properties of hydrogen culminated in the publication of this important synthesis of information. The monograph is still in demand today, although copies are rare and some information is outdated: the work will soon be available on a NIST web site. In addition to thermodynamic property tables and charts for parahydrogen and normal hydrogen (two of the important forms), there is some information on combustion properties, toxicity, radiative properties, etc. The scope of the work and the care that went into its preparation make it a key source of data even for today's efforts on the "hydrogen economy."

\begin{abstract}
The National Bureau of Standards has been engaged in the compilation, review, analytical and experimental derivation, and publication of hydrogen properties for over 20 years. The properties data presented herein are compiled largely from those accumulated data; of course, pertinent data and work of other researchers in the field are also included.

The general interests of scientists and engineers engaged in energy systems studies were given top priority in choosing the properties material presented in this book. Hydrogen systems cut across many energy related fields, e.g., nuclear fusion, magneto-hydrodynamics, electrolysis and thermochemical decomposition of water, coal and shale derivate fuels, solar and wind power, ocean energy, geothermal processes, etc. It is generally conceded that hydrogen could be used to satisfy virtually all fuel requirements that are currently being met with natural gas and oil. To satisfy the demand for properties data over this broad spectrum of interests, we have attempted to provide comprehensive coverage of physical properties over a wide range of pressures and temperatures.

Thermophysical properties of liquid, liquid-vapor, vaporous, and gaseous hydrogen are presented in Chapter 1 and the solid-liquid, solid-vapor, and solid phase properties are compiled in Chapter 2. Ortho-para modifications of the hydrogen molecule and attendant property variations are considered in both chapters. Combustion and safety data, pertinent to hazard analysis of hydrogen systems, is collected in Chapter 3. Important miscellaneous properties are compiled in Chapter 4, data figures are compiled in Chapter 5, data tables are collected in Chapter 6, and Chapter 7 summarizes symbols, units, and conversion factors used throughout the book.
\end{abstract}




\title{
A guide to the use of the ITS irregular terrain model in the area prediction mode
}

George A. Hufford, Anita G. Longley, and William A. Kissick

NTIA Institute for Telecommunication Sciences

NTIA Report 82-100, April 1982

\section{Significance}

The "Guide" describes the basis of the ITS Irregular Terrain Model (ITM or Longley-Rice Model), its implementation, and some advantages and limitations of its use. In addition, sample problems using the model in the area (site-general) prediction mode are included to demonstrate applications of the model. This classic work contains a description and implementation (version 1.2.1) of the model as it developed and matured in the years subsequent to the publication of the "Computer Method" (Longley and Rice 1968, this volume). In particular, the algorithm and its modular implementation found here represent dramatic improvements that endure to the present. In addition to providing definitive descriptions of the use of the model and its limitations, the sample problems contained here established methods of application which still guide present users. More than 20 years after this report was published, the ITS publications office receives requests for this volume almost every week.

\begin{abstract}
The ITS model of radio propagation for frequencies between $20 \mathrm{MHz}$ and $20 \mathrm{GHz}$ (the LongleyRice model) is a general purpose model that can be applied to a large variety of engineering problems. The model, which is based on electromagnetic theory and on statistical analyses of both terrain features and radio measurements, predicts the median attenuation of a radio signal as a function of distance and the variability of the signal in time and in space.

The model is described in the form used to make "area predictions" for such applications as preliminary estimates for system design, military tactical situations and surveillance, and landmobile systems. This guide describes the basis of the model, its implementation, and some advantages and limitations of its use. Sample problems are included to demonstrate applications of the model.
\end{abstract}




\title{
An attenuation function for multiple knife-edge diffraction
}

\author{
Lewis E. Vogler \\ NTIA Institute for Telecommunication Sciences
}

Radio Science, 17(6): 1541-1546, November-December 1982

\section{Significance}

For analytical and computational tractability reasons, terrain and other obstacles on the path in radio propagation problems are often approximated as a series of perfectly absorbing knifeedges. This publication presented a nearly exact expression for an arbitrary sequence of multiple (more than two) knife-edges. This was a great improvement on approximate solutions that relied on linear combinations of the single knife-edge function. In addition, it provided a series approximation to the exact expression that could be implemented in a numerically tractable fashion. Finally, the results for several sample calculations of interest were presented, along with derivations of certain closed form limiting results. Elegant and nonintuitive, this technique is the most logical way to deal with the knife-edge problem, and is considered the "gold standard" in this area.

\begin{abstract}
A recently derived attenuation function for multiple knife-edge diffraction is described, and some example calculations are presented. The function, in the form of a multiple integral, is transformed into a series representation which is amenable to computer implementation. A program has been written to compute the attenuation over propagation paths containing up to a maximum of ten knife-edges. Attenuation curves for three example paths are presented showing the effects of multiple knife-edges on the received signal. A means of estimating the statistical distribution of attenuation for a given path from statistical knowledge of the atmospheric surface refractivity is suggested.
\end{abstract}




\title{
Critical current measurements: A compendium of experimental results
}

\author{
Loren F. Goodrich and Fred R. Fickett \\ NBS Electromagnetic Technology Division
}

Cryogenics, 22(5): 225-241, May 1982

\section{Significance}

The key measurement for practical, large-scale superconductor applications is that of "critical current," the current-carrying capacity of a superconductor. Critical current is difficult to measure accurately, as demonstrated in many interlaboratory comparisons in which it is not unusual to find measurement discrepancies of up to $30 \%$. This landmark paper - published in Cryogenics, the leading journal in the field - reviewed and advanced NIST metrology for critical current. It includes an evaluation of the effect of approximately 30 experimental parameters on the critical-current determination. Everything from sample geometry and holder material to suggested uncertainties for 10 variables is described.

The paper was the prime reference in the first superconductor measurement standard in the world, published in 1982 by the American Society for Testing and Materials (ASTM). It also formed the basis for the first standard published by the International Electrotechnical Commission (IEC) in 1998. This compendium paper was also used as a guide in the late 1980s when high-temperature superconductors were discovered, and when the excitement of this discovery brought many new researchers into the field. Even with the new materials and advances that have taken place in superconductor research, the paper has stood the test of time and remains relevant to even today's best superconductors.

\begin{abstract}
The results of a program to evaluate the measurement of the critical current of relatively small $(<600 \AA)$ practical superconductors are presented. Experimental data showing the effect of various parameters on the measurement are given. Specific areas covered are: experimental design and sample mounting; electric field and resistivity criteria; temporal and spatial variations in the field and current; and temperature and strain effects. The goal of the presentation is to describe the critical current measurement process and its pitfalls in sufficient detail to serve as a guide for those relatively new to the field of practical superconductors.
\end{abstract}




\title{
Optical fiber characterization, Volumes 1 and 2
}

\author{
George E. Chamberlain, Bruce L. Danielson, Gordon W. Day, Douglas L. Franzen, Robert L. \\ Gallawa, Ernest M. Kim, and Matt Young \\ NBS Electromagnetic Technology Division
}

NBS Special Publication 637, 1982

\section{Significance}

These volumes are a collection of NBS Technical Notes that individually discuss specific areas of optical fiber characterization. The Tech Notes appeared during the commercial infancy of optical telecommunications fiber. During that period, commercially viable fibers were of the multimode type; that is, they supported the transmission along the fiber of a number of electromagnetic modes having different combinations of electric field and magnetic field configurations. While single mode fibers could be fabricated, the technology to exploit them was not readily available. Thus, this work is primarily concerned with the characterization of multimode fiber. In the early 1980s, single mode fiber became technically accessible, and today s long distance and metropolitan telecommunication networks consist solely of single mode fiber. However, multimode fiber is still used in high speed local area networks (LANs).

Fiber optic technology is measurement intensive, and multimode fibers present significant characterization problems. In the early period of optical fiber development, there were no standard measurement procedures. In a large U.S. standards effort started by the Electronic Industries Association, NBS acted as a neutral party to evaluate procedures proposed by industry. In most cases, the measurement techniques described in these volumes were, or became, industry standards. The efforts described here also provided the impetus for future NBS/NIST programs in optical fiber metrology. Fiber metrology is now a major component of the Optoelectronics Division. These volumes were identified as one of the 100 most significant NBS/NIST papers at the centennial celebration.

\begin{abstract}
Volume 1
Optical fiber waveguide measurements are described. Systems to determine the backscatter, bandwidth, and index profile are covered in detail. Measurement comparisons between laboratories are given for fiber attenuation, bandwidth, numerical aperture, and core diameter.
\end{abstract}

\begin{abstract}
Volume 2
This is the second volume of a series intended to describe optical fiber measurement systems developed at the National Bureau of Standards. The topics covered in this volume are attenuation, bandwidth (frequency domain), and far-field near-field radiation patterns. Each chapter includes a tutorial section and a detailed description of the apparatus. The volume concludes with a glossary of optical communications terms.
\end{abstract}




\title{
Design and error analysis for the WR10 thermal noise standard
}

\author{
William C. Daywitt
}

NBS Electromagnetic Fields Division

NBS Technical Note 1071, December 1983

\section{Significance}

This paper introduces the cryogenic primary noise standard, a significant improvement in the calibration of noise sources because of its high accuracy and immunity from deterioration that is common in oven-based noise standards. The cryogenic noise standard described in this Technical Note is still in use today at NIST. As well, many modern commercially available primary noise standards are based on the NBS design presented here.

\begin{abstract}
This note describes the design and error analysis of a WR10 thermal noise power standard. The standard is designed to operate at the boiling point of liquid nitrogen with a noise temperature accurate to $\pm 1 \mathrm{~K}$.
\end{abstract}




\section{Direct frequency measurement of the I2-stabilized He-Ne 473-THz (633-nm) laser}

Donald A. Jennings, Clifford R. Pollock, F. Russell Petersen, Robert E. Drullinger, Kenneth M. Evenson, and Joseph S. Wells

NBS Time and Frequency Division

John L. Hall

NBS Quantum Physics Division

Howard P. Layer

NBS Length and Mass Measurement and Standards Division

Optics Letters 8: 136-138, March 1, 1983

\section{Significance}

This paper was a crucial part of research to improve the measurement of the speed of light, which then led to international redefinition of the meter. The original definition of the meter was based on an artifact, a precision "ruler" maintained at the International Bureau of Weights and Measures in France. In 1960, the meter was redefined based on the wavelength of an optical transition in the atom krypton. But rapid improvements in laser technology after its 1961 introduction led to new high precision measurements of the speed of light, the meter, and frequency, which are all interrelated. This paper reported exquisitely accurate measurements of a wide range of frequencies, all directly interlinked, which reduced the uncertainty in the speed of light by a factor of 100 . With this improvement, it became clear that the meter could be much more accurately defined by basing it on the speed of light, and in 1983 the international agreement became: "The meter is the length of the path traveled by light in vacuum during a time interval of 1/299 792458 of a second," with the speed of light taken as a defined fundamental constant. Note that this redefinition makes the meter dependent on the measurement of time (the second), which is by far the most accurately realized of the seven fundamental units.

\footnotetext{
Abstract

The absolute frequency of the 473-THz He-Ne laser $(633 \mathrm{~nm})$, stabilized on the $g$ or $i$ hyperfine component of the ${ }^{127} \mathrm{I}_{2}$ 11-5 R(127) transition, was measured by comparing its frequency with a known frequency synthesized by summing the radiation from three lasers in a He-Ne plasma. The three lasers were (1) the 88- $\mathrm{THz} \mathrm{CH}_{4}$-stabilized He-Ne laser $(3.39 \mu \mathrm{m})$, (2) a $125-\mathrm{THz}$ color-center laser $(2.39 \mu \mathrm{m})$ with its frequency referenced to the $\mathrm{R}_{\mathrm{II}}(26){ }^{13} \mathrm{C}^{18} \mathrm{O}_{2}$ laser, and (3) the $260-\mathrm{THz} \mathrm{He}-\mathrm{Ne}$ laser $(1.15 \mu \mathrm{m})$ referenced to an $\mathrm{I}_{2}$-stabilized dye laser at $520 \mathrm{THz}(576 \mathrm{~nm})$. The measured frequencies are 473612340.492 and $473612214.789 \mathrm{MHz}$ for the $g$ and $i$ hyperfine components, respectively, with a total uncertainty of 1.6 parts in $10^{10}$. The frequency of the $i$ component adjusted to the operating conditions recommended by the Bureau International des Poids et Mesures is $473612214.830 \pm 0.074 \mathrm{MHz}$.
} 


\title{
Large-scale meteorological conditions associated with midlatitude, mesoscale convective complexes
}

\author{
Robert A. Maddox \\ NOAA Weather Research Program \\ Monthly Weather Review, 111: 1475-1493, 1983
}

\section{Significance}

After establishing quantitative criteria that essentially defined Mesoscale Convective Complexes (MCCs), here Maddox describes the large-scale settings in which MCCs form. His work inspired a large body of further research, including yearly summaries of MCC occurrences in the United States, a (successful) search for MCCs on other continents, examinations of the hydrologic significance of the severe weather produced in MCCs, and modeling studies intent on setting out the physical mechanisms driving these large mesoscale circulations. The concept of MCCs helps to explain several other phenomena, e.g., the nocturnal maximum of warm season rainfall observed over much of the central and northern US. It has also initiated continued debate over whether MCCs are distinct meteorological phenomena with an inherent scale, or if they are simply one of many on a continuum of scales between convective cells and short waves. Warmseason precipitation forecasting has been strongly influenced by the understanding of MCCs, enabling forecasters to put the large anvil features seen on satellite imagery in context.

\begin{abstract}
Objective analyses of composited meteorological conditions attending ten Mesoscale Convective Complexes (MCC) reveal a number of distinctive characteristics and important interactions with the large-scale environment. The systems appear to be linked to eastward progression of a weak, middle-tropospheric, short-wave trough. Initial thunderstorms develop within a region of mesoscale convergence and lifting that is apparently forced primarily by low-level warm advection. The MCC system acquires mesoscale organization while it moves eastward ahead of the short-wave trough. Diabatic heating eventually produces a system that is warm core in the middle troposphere and cold core in upper levels. The mature MCC exhibits many similarities to tropical convective systems, although it occurs within a considerably different large-scale setting. Inflow within the lower half of the troposphere feeds convection within a region characterized by significant net upward mass flux and widespread precipitation. Thickness increases within this region produce anomalously high heights in the upper-troposphere above the MCC and intense outflow develops in the region where the height gradient has increased. Decay typically occurs as the system moves east of the region of conditionally unstable air and low-level warm advection. However, as the system decays, atmospheric response to residual temperature perturbations results in intensification of the precursor short-wave trough within the upper half of the troposphere.
\end{abstract}




\title{
Liquefied natural gas densities: Summary of research program at the National Bureau of Standards
}

\author{
William M. Haynes, Robert D. McCarty, and Michael J. Hiza \\ NBS Chemical Engineering Science Division
}

NBS Monograph 172, 1983

\section{Significance}

The program in the Boulder Laboratories on the properties of liquefied natural gas (LNG) provided the core metrology to allow custody transfer-commerce-in this key commodity. The properties of LNG vary, by composition or source, temperature, and pressure, and transport of this fuel often involves mixing, cooling, warming, pressurizing and venting. It is important to know the fluid properties at each stage of the process. Monograph 172 summarizes the measurements made in Boulder as well as models for describing LNG fluids - and an important sector of our energy economy is based on the information in this monograph. A one percent error in density, which was typical of the state of the art for LNG commerce at the inception of this project, corresponds to an inequity of a quarter of million dollars in the assessed value of a single shipload of LNG.
Abstract
This report summarizes the results of a project concerning the densities of liquefied natural gas (LNG) and its components. This project, initiated in the Properties of Fluids Section of the Cryogenics Division of the National Bureau of Standards in July 1972, was carried out under the sponsorship of a consortium of 18 energy companies.
The experimental part of this project has included the following accomplishments: (a) development of a magnetic suspension densimeter for absolute density measurements on liquids, including liquid mixtures in equilibrium with their vapor, at temperatures from 90 to $300 \mathrm{~K}$; (b) orthobaric liquid density measurements on the major components of LNG, which include nitrogen $(95-120 \mathrm{~K})$, methane $(105-160 \mathrm{~K})$, ethane (100-270 K), propane (100-288.7 K), isobutane $(115-300 \mathrm{~K})$, and normal butane (135-300 K); (c) orthobaric liquid density measurements on approximately 35 binary mixtures of the above components for all combinations except nitrogen + butane systems, primarily in the temperature range of 105 to 130 $\mathrm{K}$; and (d) orthobaric liquid density measurements on 27 multicomponent mixtures (105-120 K), including several LNG-like mixtures with up to 8 components. The total uncertainty of a single density measurement is approximately 0.1 percent at low temperatures and decreases to approximately 0.06 percent at room temperature. The estimated standard deviation of a single density measurement is less than 0.02 percent.

The density data have been used to optimize, test, and compare several mathematical models as to their suitability for the calculation of LNG densities for custody transfer. Models selected for 
optimization and testing included an extended corresponding states method, a hard sphere model, a cell model, and an empirical model due to Klosek and McKinley. The ultimate goal of this project was to produce one or more mathematical models that could be used to predict the density of any LNG mixture to within an uncertainty of 0.1 percent from an input of pressure, temperature, and composition. After revisions based on the new experimental data from this project, each of the models investigated here satisfy this goal for typical LNG compositions. The limitations and ranges of validity of the various models are discussed. Also presented are techniques for predicting LNG densities from dielectric constant measurements and from excess volume calculations.

The last section of this report consists of publications that provide a complete and detailed account of the results of this project. 


\title{
Materials at low temperatures
}

\author{
Richard P. Reed and Alan F. Clark, editors \\ NBS Fracture and Deformation Division
}

American Society for Metals, 1983, 590 pp.

\section{Significance}

This book summarizes about 30 years of data and experience developed within the Cryogenics Division of NBS. Most of the chapters are on categories of properties such as: elastic properties, specific heat, thermal expansion, thermal conductivity, electrical properties, magnetic properties, mechanical properties, and fracture mechanics. Other chapters look at particular materials classes such as structural alloys, composites, and superconductors.

The book describes the division's support of the industries that required low-temperature data and materials. Study of the technical roadblocks led to both new materials (cryogenic structural alloys with higher strength and higher toughness, better insulators, and improved superconductors) and improved procedures and designs (lower loss Dewars, reduction in thermal stresses through balancing of material expansion, quantitative design assessment techniques, and test procedures that reflect new parameters).

Abstract (since the book has no abstract, a selection from the Preface is given here)

... The thirteen contributors to this book formed the nucleus of the materials group of the Cryogenics Division of the National Bureau of Standards (NBS). The Division was formed in 1952 as part of the expansion of NBS to their new Boulder (Colorado) Laboratories, and our group was composed of specialists in physical and mechanical properties of solids at low temperatures. We primarily served emerging low-temperature technologies requiring cryogenic materials, such as space, LNG storage and transportation, magnetohydrodynamic energy conversion, fusion, high-energy physics, and other aspects of applied superconductivity. We evolved into the largest materials research group in the free world devoted to low-temperature research.

We have now dispersed into several disciplines, but most of our low-temperature research capabilities and interests remain. While these are still strong, we thought it appropriate to summarize our data and experience in this book. We hope our contributions will establish a firm basis for the understanding of materials behavior and property measurement to use for design and material selection at low temperatures .... 


\title{
The Colorado wind-profiling network
}

\author{
Richard G. Strauch, David A. Merritt, Kenneth P. Moran, Kenneth B. Earnshaw, and \\ Douglas van de Kamp \\ NOAA Wave Propagation Laboratory
}

Journal of Atmospheric and Oceanic Technology, 1(1): 37-49, 1984

\section{Significance}

This paper describes the work that led to the deployment of the NOAA Wind Profiling Network that was implemented in 1989 and continues to provide real-time wind data today. The techniques developed for the Colorado Wind-Profiling Network formed the basis for the original specifications to industry for procurement of the NOAA profilers. The wind profiling method described in this paper is still how Doppler wind profilers are operated worldwide. This work built on research conducted in the Aeronomy Lab and subsequently transferred to the Forecast Systems Lab. The Colorado network comprised VHF radars and the first UHF wind profiler radar. This latter was crucial for showing that wind profilers could be operated at higher frequencies where frequency authorizations were possible and with smaller antennas where sites were more readily available.

The development of the Doppler wind profiler technology within the Boulder Labs has resulted in significant impacts to the nation, and the world, primarily in improved weather forecasts and safety of life and property. This technology represents the most significant advancement in continuous, all-weather, real-time atmospheric wind measurements in the past 50+ years. NOAA and the National Weather Service distribute the data worldwide for use by many other meteorological agencies. Data from the NOAA Network have even been shown to improve weather forecasts in Europe. In addition, economic, health, and additional safety benefits have been realized, including new manufacturing/repair activities that developed in support of the wind profiling technology, improved aviation safety and efficient commercial aircraft routing, and Space Shuttle and other rocket launch/landing support, among others. This paper has been referenced 94 times in journal articles, and across multiple disciplines, including radio science, geophysics, meteorological research, operational meteorology, and optics.

\begin{abstract}
Remote sensing instrumentation has advanced to the point where serious consideration is being given to a next-generation tropospheric sounding system that uses radars and radiometers to provide profiles of tropospheric variables continuously and automatically. A network of five wind-profiling radars has been constructed in Colorado. This network represents a significant step in the development of a new observing system for operational and research meteorology. The radars and their capabilities and limitations are described.
\end{abstract}




\title{
A practical Josephson voltage standard at $1 \mathrm{~V}$
}

\author{
Clark A. Hamilton, Richard L. Kautz, and Frances L. Lloyd \\ NBS Electromagnetic Technology Division
}

IEEE Electron Device Letters, EDL-6: 623-625, 1985

\section{Significance}

The Josephson junction was officially adopted as the basis for precise voltage measurement in 1972. While early Josephson standards provided the first reference voltage that was stable over long periods, they were difficult to use because the voltage generated was only a few millivolts. In 1985, Clark Hamilton, Richard Kautz, and Frances Lloyd introduced a new type of Josephson voltage standard based on a series array of 1,484 junctions fabricated on a single microchip. The original array could be adjusted to produce a variety of voltages up to one volt, and later versions with over 14,000 junctions yielded up to $10 \mathrm{~V}$. Array standards have revolutionized voltage measurement, making it easier and more accurate, and are now used in government and industrial laboratories around the world.

\begin{abstract}
A series array of 1484 pairs of Josephson junctions, biased by microwaves at $72 \mathrm{GHz}$, is demonstrated to provide stable quantized voltages at the $1 \mathrm{~V}$ level. The niobium/lead-alloy junctions used in the array are not affected by thermal cycling.
\end{abstract}




\title{
Electron-tunneling experiments using Nb-Sn "break" junctions
}

\author{
John Moreland and Jack W. Ekin \\ NBS Electromagnetic Technology Division
}

Journal of Applied Physics, 58: 3888-3895, November 1985

\section{Significance}

Break junctions, the subject of this paper, were originally developed at NIST for tunneling spectroscopy studies of certain superconductors to measure energy gap and phonon coupling. These materials were difficult to prepare in thin-film form for conventional tunnel junction measurements. Several authors have subsequently expanded on break junctions to include research on atomic wires and molecular electronics, taking advantage of the adjustable subatomic positioning capabilities of the technique. Following the seminal work at NIST, 295 articles have referenced break junctions in their abstracts, employing concepts originally put forth in the 1985 NIST article. Over 50 of these cite the NIST work directly. Tunneling spectroscopy and tunnel-junction technology research and theory has led to several Nobel prizes.

\begin{abstract}
$\mathrm{An} \mathrm{Nb}-\mathrm{Sn}$ filament mounted on a flexible glass beam can be broken to form an electron tunneling junction between the fracture elements. Breaking the filament in liquid helium prevents oxidation of the freshly exposed fracture surfaces. A sharp superconducting energy gap in the $I-V$ characteristics measured at $4 \mathrm{~K}$ indicates the formation of a high-quality tunneling barrier between the fracture elements. The resistance of the junction can be continuously adjusted by varying the surface bending strain of the beam. An estimated $0.1 \mathrm{~nm}$ change in the barrier thickness produces about an order of magnitude change in the resistance over the range from $10^{5}$ to $10^{8} \Omega$. The exponential character of this dependence shows that the tunnel junction is freely adjustable without intimate contact of the junction elements. "Break" junctions made in this way offer a new class of tunneling experiments on freshly exposed surfaces of a fractured sample without the oxide barrier previously required for junction stability. Such experiments provide a simple technique for tunneling to new materials and may eliminate complications that can be encountered during interpretation of data obtained using oxide barriers.
\end{abstract}




\title{
A comparison of three types of pulse tube refrigerators: New methods for reaching $60 \mathrm{~K}$
}

Ray Radebaugh, James Zimmerman, David R. Smith, and Beverly Louie

NBS Chemical Engineering Science Division

Advances in Cryogenic Engineering, Vol. 31, pp. 779-789, 1986

\section{Significance}

This paper, published in the proceedings of the 1985 Cryogenic Engineering Conference, presented the first results of achieving temperatures below $100 \mathrm{~K}$ with a new type of refrigerator that was first proposed in Moscow two years earlier. It won the best paper award at the conference. This work started a worldwide interest in pulse tube refrigerators, which have become the most efficient cryocoolers for temperatures in the range of $60 \mathrm{~K}$ to $100 \mathrm{~K}$. These refrigerators are now commercially available and are replacing many other types of cryocoolers for a wide variety of applications. They have become the most common type of cryocooler to use for space applications by the Air Force and by NASA. Many authors of papers on pulse tube refrigerators reference this paper.

\begin{abstract}
Pulse tube or thermoacoustic refrigerators require only one moving part—an oscillating piston or diaphragm at room temperature. Refrigeration occurs within a tube connected to the pressure wave generator when the thermal relaxation time between gas and tube is comparable to a half period. Three types have been discussed in the literature recently by Gifford, by Mikulin, and by Wheatley. A record low temperature of $60 \mathrm{~K}$ was achieved in our work using a single stage pulse tube similar to that of Mikulin. Previously $105 \mathrm{~K}$ was the lowest temperature achieved. Because of only one moving part, all three types have the potential for long life, but their efficiency and intrinsic limitations have never been investigated. This paper compares the three types with each other and with common refrigerators such as Joule-Thomson and Stirling refrigerators. An apparatus is described which can measure the intrinsic behavior of the different types from temperatures of about $30 \mathrm{~K}$ to $300 \mathrm{~K}$. Overall cycle efficiency as well as sources of loss such as conduction and regenerator ineffectiveness are discussed and the advantages of various phase shifting techniques to increase refrigeration capacity are compared.
\end{abstract}




\title{
On the depletion of Antarctic ozone
}

\author{
Susan Solomon \\ NOAA/CIRES Aeronomy Laboratory \\ Rolando R. Garcia \\ National Center for Atmospheric Research \\ F. Sherwood Rowland \\ Department of Chemistry, University. of California, Irvine \\ Donald J. Wuebbles \\ Lawrence Livermore National Laboratory
}

Nature, 321(6072): 755-758, 1986

\section{Significance}

In this paper, Dr. Solomon proposed what turned out to be the correct theory of the cause of the Antarctic ozone hole and propelled international efforts to protect the ozone layer.

When the Antarctic ozone hole was discovered in 1986 by the British Antarctic Survey, theories abounded as to its cause. Dynamical, chemical, and solar arguments were made. This paper by Solomon et al. pointed out that human-produced chlorine compounds could be interacting with stratospheric ice clouds and that, in the meteorological setting that is unique to the polar regions, the combination of conditions could produce extreme ozone losses. It was a remarkable insight, one that required thinking "outside the box" of any one discipline in the atmospheric sciences. Chemistry, dynamics, and seasonal meteorology were all critical aspects of the theory. And as it turned out, the authors were right. Later observational evidence, gathered by an international team of researchers in the National Ozone Expeditions of 1986 and 1987 led by lead author Solomon, confirmed the theory advanced by this landmark 1986 Nature paper. This paper and the subsequent confirmation of its theory have literally launched a new area of research in atmospheric science: the study of chemical reactions occurring on the surfaces of particles. Prior to the discovery of the ozone hole, this so-called "heterogeneous chemistry" was a fledgling area of stratospheric research (a fact that explains why the ozone hole caught the entire research community off guard!). Today, the field is transformed and heterogeneous chemistry is recognized as a pivotal factor in ozone depletion as well as other atmospheric issues. Lead author Solomon was awarded the 1999 National Medal of Science by President Clinton in recognition of her achievement, and coauthor Rowland received the Nobel Prize for this and other work on the ozone layer.

\begin{abstract}
Recent observations by Farman et al. reveal remarkable depletions in the total atmospheric ozone content in Antarctica. The observed total ozone decreased smoothly during the period from about 1975 to the present, but only in the spring season. The observed ozone content at Halley Bay was $\sim 30 \%$ lower in the Antarctic spring seasons (October) of 1980-84 than in the springs of 195773. No such obvious perturbation is observable in other seasons, or at other than the very highest
\end{abstract}


latitude in the Southern Hemisphere, and the magnitude of the observed change there far exceeds climatological variability. We present here balloonsonde ozone data which show that these ozone changes are largely confined to the region from about 10 to $20 \mathrm{~km}$, during the period August to October. We show that homogeneous (gas phase) chemistry as presently understood cannot explain these observed depletions. On the other hand, a unique feature of the Antarctic lower stratosphere is its high frequency of polar stratospheric clouds, providing a reaction site for heterogeneous reactions. A heterogeneous reaction between $\mathrm{HCl}$ and $\mathrm{ClONO}_{2}$ is explored as a possible mechanism to explain the ozone observations. This process produces changes in ozone that are consistent with the observations, and its implications for the behaviour of $\mathrm{HNO}_{3}$ and $\mathrm{NO}_{2}$ in the Antarctic stratosphere are consistent with observations of those species there, providing an important check on the proposed mechanism. Similar ozone changes are obtained with another possible heterogeneous reaction, $\mathrm{H}_{2} \mathrm{O}+\mathrm{ClONO}_{2}$. 


\section{Reducing the risk of refinery explosions:}

\section{Examination of a pressure vessel that ruptured at the Chicago refinery of the Union Oil Company on July 23, 1984}

Harry I. McHenry, T. Robert Shives, David T. Read, J. David McColskey, Charles H. Brady, and Patrick T. Purtscher

NBS Fracture and Deformation Division

NBSIR 86-3049, 1986

\section{Observations of hydrogen damage in a failed pressure vessel}

Harry I. McHenry, Patrick T. Purtscher, and T. Robert Shives

NBS Fracture and Deformation Division

Corrosion Science, 27(10/11): 1041-1057, 1987

\section{Failure analysis of an amine-absorber pressure vessel}

Harry I. McHenry, David T. Read, and T. Robert Shives

NBS Fracture and Deformation Division

Materials Performance, 26(8): 18-24, August 1987

\section{Background}

In July 1984, an explosion and fire occurred at the Chicago Refinery of Union Oil Company, killing 17 people and causing extensive property damage. NBS was requested by the Occupational Safety and Health Administration (OSHA) to conduct an investigation into the failure of the pressure vessel that eyewitnesses identified as the initial source of the explosion and fire. Sections of the vessel were shipped to NBS in August 1985, where a multi-disciplinary team, led by Harry McHenry, the Deputy Chief of the Fracture and Deformation Division, sought the cause of the failure. Twenty-three NBS staff members participated in the study, from both Boulder and Gaithersburg.

\section{Summary of the primary publication}

The study's findings were published as NBSIR 86-3049. This report outlined the history of the vessel and described the steps leading up to its failure. The investigation involved examination of the vessel pieces, material property tests, corrosion and hydrogen cracking studies, fracture surface observations, and a fracture mechanics assessment. The investigation determined that the vessel fractured along a path weakened by extensive cracking adjacent to a repair weld. These pre-existing cracks grew through the vessel wall due to hydrogen pressure cracking. When the 
depth of the largest of these cracks exceeded 90 to $95 \%$ of the wall thickness, the remaining thin ligament of steel in the cracked section ruptured and leakage occured. This crack triggered a complete fracture of the vessel circumference at the operating stress level of only $35 \mathrm{MPa}$ because the toughness of the vessel steel had been reduced by hydrogen embrittlement. The publication identified previously unrecognized interactions between pressure vessel steel, the thermal cycles that occur during repair welds, and hydrogen-containing environments.

Rapid dissemination of the information was important because other vessels might also have been at risk of catastrophic failure. In 1986, the report findings were summarized in articles in Corrosion Science and Materials Performance, journals that are read by industrial process engineers and safety officials in these industries.

\section{Short-term impact}

OSHA recognized the technical complexities involved in assessing the mechanical integrity of the various items of equipment used in these industries. To provide OSHA inspectors and regulators with the technical information needed, OSHA engaged NIST to perform two additional tasks:

- Prepare an information document titled: "Guidelines for Pressure Vessel Safety Assessment" for distribution within OSHA, and

- Organize and conduct a training course, Course 340 (Hazard Analysis in the Chemical Processing Industries), for OSHA inspectors with information lectures concerning pressurized equipment. The first course was taught at Boulder in 1988.

In addition, the reporting of the findings in Corrosion Science and Materials Performance allowed industrial process engineers and inspection personnel to update the inspection plans for plants under their control. Now that they were aware of the conditions found in this study, the inspection personnel could search for similar problems (such as hydrogen blisters and cracks) during the next annual inspections or shutdowns of processing plants under their control, and inspection intervals could be adjusted accordingly.

\section{Long-term impact}

Several years following the NIST report, OSHA issued a final rule titled "Process Safety Management of Highly Hazardous Chemicals, Explosives and Blasting Agents." This rule was published as 29CFR Part 1910 in the Federal Register on February 24, 1991, and became effective on May 26, 1992. These rules have had a major impact on the process industry; particularly those parts that indicated for example, that inspection and testing procedures to assess the mechanical integrity of process equipment shall follow recognized and generally good engineering practices. These rules have prompted cooperatively funded activities in the process and allied industry sectors that focused on the preparation of technical documents that describe and define methodology, procedures, and techniques that constitute "recognized and good engineering practices." The first document developed in this activity is expected to be available in early 2000. Meanwhile, the OHSA training center continues to offer the training course on hazard analysis to its inspectors. It has been held 11 times, and about 230 inspectors have attended. 


\title{
Models and observations of the impact of natural hydrocarbons on rural ozone
}

Michael Trainer and Eric J. Williams

NOAA Aeronomy Laboratory/CIRES

David D. Parrish

Department of Chemistry

Metropolitan State College

Martin P. Buhr

NOAA Aeronomy Laboratory/CIRES

Nature, 329(6141): 705-707, 1987
Eugene J. Allwine and Halvor H. Westberg

Laboratory for Atmospheric Research

Washington State University

Fred C. Fehsenfeld and Shaw C. Liu

NOAA Aeronomy Laboratory

\section{Significance}

Air pollution, or "smog" consists of elevated amounts of ozone, fine particles, and other trace species near the earth's surface. Ozone is formed in the atmosphere by the interaction of nitrogen oxides, hydrocarbons, and sunlight. However, in some parts of the country, attempts to improve air quality by regulating human-caused emissions of hydrocarbons have been unsuccessful. This paper demonstrated that natural sources of hydrocarbons (trees and other vegetation) can be so large that human emissions are (relatively) insignificant. The results suggested that controlling human emissions of other compounds, e.g., the nitrogen oxides, would be more effective in reducing surface-level ozone pollution. Subsequent research has identified regions in the country, primarily in the southeastern U.S., where natural hydrocarbons are more abundant than had been recognized. The use of scientifically customized approaches to air quality management has led to greater improvements in air quality for many regions of the nation.

\begin{abstract}
Large quantities of non-methane hydrocarbons (NMHCs) are emitted into the atmosphere from vegetation. A recent inventory of Lamb et al., indicates that the emissions of natural hydrocarbons is significant compared to that of anthropogenic NMHCs in most regions of the United States. Because of their chemical activity, the natural NMHCs can play important parts in the formation of trace gases, such as ozone $\left(\mathrm{O}_{3}\right)$, peroxyacetyl nitrate $(\mathrm{PAN})$ and oxygenated secondary hydrocarbons, which contribute to regional-scale air pollution and may be harmful to crops and forest. The impact of natural hydrocarbons on the formation of $\mathrm{O}_{3}$ in the atmosphere has been discussed previously. In general, it has been concluded that their impact is small. But lack of data on the ambient concentrations of key photochemical species and an incomplete analysis of the photochemistry has prevented a definitive evaluation of the impact of natural $\mathrm{NMHCs}$ on rural $\mathrm{O}_{3}$. Here we report on concentrations of key trace gases measured concurrently at a rural site in the eastern USA during the summer of 1986 and a modelling study conducted to analyze these measurements. This study demonstrated that natural NMHCs can have a significant impact on ozone formation in rural air.
\end{abstract}




\title{
Second viscosity and thermal conductivity virial coefficients of gases: Extension to low reduced temperature
}

\author{
James C. Rainwater and Daniel G. Friend \\ NBS Thermophysics Division
}

Physical Review A, 36: 4062-4066, 1987

\section{Significance}

The ability to predict the properties of fluids from first principles, whether using quantum mechanics or classical approaches, remains an elusive goal. Some rigorous results are available, but a systematic approach based on density expansions - a virial series - had not been successful for transport properties. This paper showed how to account for particle interactions in the first transport property virial coefficients, even at low reduced temperature. It was published in part to explain some recent (1986) measurements on the viscosity of two specific vapors (benzene and methanol), but has since been used as the basis for describing the viscosity surface of many vapors. The theoretical techniques have evolved into practical methods of describing empirical data, and the ideas have now been incorporated in several international standards.

\begin{abstract}
A recent theory of the initial density dependences of both viscosity and thermal conductivity has been extended to include lower reduced temperatures. New data on the second viscosity virial coefficients of some organic vapors are found to be in substantial agreement with the theory even at the lowest temperatures. We present in tabular form the numerical values for both transport virial coefficients in the reduced temperature range $0.5 \leq \mathrm{T}^{*} \leq 100$ and include values for the constituent two-monomer, three-monomer, and monomer-dimer contributions. A brief discussion of the theoretical approach and calculational methods is also given.
\end{abstract}




\title{
Tunneling spectroscopy of a La-Sr-Cu-O break junction: Evidence for strong coupling superconductivity
}

\author{
John M. Moreland, Alan F. Clark, and Loren F. Goodrich \\ NBS Electromagnetic Technology Division \\ Harry C. Ku and R.N. Shelton \\ Ames Laboratory and Physics Department \\ Iowa State University, Ames, Iowa
}

Physical Review B 35(16): 8711-8713, June 1987

\section{Significance}

This is the report of the first observation and measurement of the energy gap in a high critical temperature superconductor (HTS). These unique materials were discovered by the Nobel Prize winners, Bednorz and Mueller, in late 1986 and first revealed in backroom discussions at the Materials Research Society meeting in December 1986. With just a few materials urgently developed by colleagues at Iowa State University (the coauthors Ku and Shelton), this group of NBS researchers quickly adapted a measurement technique developed by John Moreland to make the first determination of the superconducting energy gap in any HTS material. This established the certainty of superconductivity and determined the nature and magnitude of this most important physical parameter of superconductivity. The authors were selected to be some of the few to report their measurements at the now famous 1987 "March Meeting" of the American Physical Society where thousands of physicists literally scrambled over each other for seats to hear these exciting results. This Physical Review article summarizes the verbally reported results and outlines their relevance to the now accepted high temperature superconductors. The work led to a DOC Silver Medal and APS Fellowship.

\begin{abstract}
Detailed structure in the quasiparticle tunneling has been observed in $\mathrm{La}-\mathrm{Sr}-\mathrm{Cu}-\mathrm{O}$ superconductive tunneling junctions using the break-junction technique. Variability in the energy gap and associated structure in the current-voltage curves are observed indicating significant inhomogeneity in the superconducting properties. Large energy gaps $(7.0 \mathrm{meV})$ and deep structure in the conductance derivative are evidence for a strong-coupling mechanism.
\end{abstract}




\title{
Error analysis techniques for planar near-field measurements
}

\author{
Allen C. Newell \\ NBS Electromagnetic Fields Division \\ IEEE Transactions on Antennas and Propagation AP-36: 754-768, June 1988
}

\section{Significance}

This work is the model from which most uncertainty evaluations of near-field measurements are derived. The paper received the "S.A. Schelkunoff Award" in 1989 and the "W.R.G. Baker Award" in 1990. These are the best paper awards of the IEEE Antennas and Propagation Society and the overall IEEE, respectively.

\begin{abstract}
The results of an extensive error analysis on planar near-field measurements are described. The analysis provides ways for estimating the magnitude of each individual source of error and then combining them to estimate the total uncertainty in the measurement. Mathematical analysis, computer simulation, and measurement tests are all used where appropriate.
\end{abstract}




\title{
Josephson-junction model of critical current in granular $\mathrm{Y}_{1} \mathrm{Ba}_{2} \mathrm{Cu}_{3} \mathrm{O}_{7-\delta}$ superconductors
}

\author{
Robert L. Peterson and Jack W. Ekin \\ NBS Electromagnetic Technology Division
}

Physical Review B, 37: 9848-9851, June 1988

\section{Significance}

While ceramic high-temperature superconductors (HTS) were being intensely investigated in the late 1980s, it seemed that their transport critical currents were strongly affected by "weak links" that blocked the flow of supercurrents at very low magnetic fields. However, no quantitative theory existed to explain the experimental evidence and support the hypothesis. Peterson and Ekin presented the first quantitative evidence that identified grain-boundary weak links as the source of the transport current problem. This often-cited paper showed that all earlier experimental results-including the pioneering measurements of Ekin et al. (Journal of Applied Physics, vol. 62, pp. 4821-4828, 1987) — could, in fact, be quantitatively explained in terms of a grain-boundary Josephson-tunneling weak-link model with no adjustable parameters. The grainboundary weak-link effect has dominated much of the research on HTS materials ever since. It has become the principal consideration in the development of practical HTS conductors

\begin{abstract}
We calculate the transport critical-current density in a granular superconductor in magnetic fields below about $5 \times 10^{-3} \mathrm{~T}$. The field dependence in this region is assumed to be controlled by intragranular or intergranular Josephson junctions. Various model calculations are fitted to transport critical-current data on bulk $\mathrm{Y}_{1} \mathrm{Ba}_{2} \mathrm{Cu}_{3} \mathrm{O}_{7-\delta}$ ceramic superconductors, whose average grain size somewhat exceeds $10 \mu \mathrm{m}$. The results yield an average junction cross-sectional area (thickness $\times$ length) of 4-6 $\mu \mathrm{m}^{2}$. If the junctions are at the grain boundaries, a London penetration depth of about 150-300 nm is inferred, consistent with other estimates. We conclude that Josephson junctions are limiting the transport current in these samples and that they lie at the grain boundaries. The parameters of the fit are not consistent with Josephson junctions at twinning boundaries.
\end{abstract}




\title{
Handbook of basic tables for chemical analysis
}

\author{
Thomas J. Bruno and Paris D.N. Svoronos \\ NIST Thermophysics Division
}

CRC Press, Boca Raton, FL, 1989

\section{Significance}

This important handbook, whose second edition was published in 2003, is one of the most highly cited references in the field of chemical analysis. The book was an outgrowth of materials prepared for a course on chemical instrumentation presented in the Boulder Laboratories, and covers the classical fields of chromatography, spectroscopy, and chemical (wet) methods. The tables, notes, spectra, and references in this handbook provide a compact and complete set of information used in analytical laboratories throughout the world.

Abstract (since this book has no abstract, an excerpt from the Preface is included)

... Although there have been considerable changes since the first version of the tables, the aim has remained essentially the same. We have tried to provide a single source of information for those practicing scientists and research students who must use various aspects of chemical analysis in their work. In this respect, it is geared less toward the researcher in analytical chemistry than to those practitioners in other chemical disciplines who must make routine use of chemical analysis. We have given special emphasis to those "instrumental techniques" which are most useful in solving common analytical problems. In many cases, the tables contain information gleaned from the most current research papers and provide data not easily obtainable elsewhere. In some cases, data are presented which are not available at all in other sources. An example is the section covering supercritical fluid chromatography, in which a tabular P- $\rho-\mathrm{T}$ surface for carbon dioxide has been calculated (specifically for this work), using an accurate equation of state .... 


\title{
MPM-An atmospheric millimeter-wave propagation model
}

\author{
Hans J. Liebe \\ NTIA Institute for Telecommunication Sciences \\ International Journal of Infrared and Millimeter Waves, 10 (6): 631-650, 1989
}

\section{Significance}

This paper is one of the most inclusive publications of a body of work that produced measurements and models of millimeter wave propagation that remain today unmatched in accuracy. This article introduces MPM, a model for complex refractivity of a clear atmosphere below $1 \mathrm{THz}$. It incorporates the quantum effects of molecular oxygen and other atmospheric constituents to describe propagation effects (delay and loss) and their dependencies on temperature, pressure, humidity, and composition. This work defined atmospheric absorption for a broad piece of the radio spectrum for such applications as remote sensing technology. Fifteen years after this work was published, MPM is still considered an international standard and is widely used.

\begin{abstract}
A broadband model for complex refractivity is presented to predict propagation effects of loss and delay for the neutral atmosphere at frequencies up to $1000 \mathrm{GHz}$. Contributions from dry air, water vapor, suspended water droplets (haze, fog, cloud), and rain are addressed. For clear air, the local line base ( $44 \mathrm{O}_{2}$ plus $30 \mathrm{H}_{2} \mathrm{O}$ lines) is complemented by an empirical water-vapor continuum. Input variables are barometric pressure, temperature, relative humidity, suspended water droplet concentration, and rainfall rate.
\end{abstract}




\title{
Performance standards for packet-switched services
}

\author{
Neal B. Seitz
}

NTIA Institute for Telecommunication Sciences

Proceedings of the IEEE International Communications Conference, 63-70, June 1989

\section{Significance}

This paper describes a family of CCITT (now ITU-T) Recommendations, developed under NTIA/ITS leadership, that collectively standardize performance metrics and worst-case performance values for international packet-switched services using the X.25/X.75 protocols. The standard metrics have been implemented in network management systems and stand-alone test equipment and the recommended performance values have been used in planning, designing, operating, and maintaining first generation packet switched networks in many countries. The performance specification and measurement concepts described in the paper provided the framework for over a dozen subsequent national and international data network performance standards, including, most recently, an ITU-T Recommendation defining standard Quality of Service (QoS) classes for IP-based networks.

\begin{abstract}
Four new Recommendations on the performance of international packet-switched services were developed in CCITT Study Group VII during the 1985-1988 Study Period. Recommendation $X .134$ defines apportionment boundaries and packet layer reference events which provide a basis for performance description. The boundaries are physical interfaces at which CCITT Recommendation $X .25$ or $X .75$ procedures are used, and the events are corresponding interface state transitions. Recommendations $X .135, X .136$, and $X .137$ define specific packet-switched service performance parameters and associated values. The parameters are measures of packetswitched service speed, accuracy, dependability, and availability applicable to end-to-end virtual connections and their component sections. The values are worst-case performance limits for the national and international portions of an international virtual connection. It is anticipated that these performance parameters and values will be widely used in the planning and operation of packet-switched services supporting international data communications. The approach used in developing the packet-switched service parameters has been adapted for use in developing performance parameters for Integrated Services Digital Networks (ISDNs).
\end{abstract}




\title{
Stainless steel weld metal: Prediction of ferrite content
}

\author{
Chris N. McCowan, Thomas A. Siewert, and D.L. Olson \\ NIST Materials Reliability Division
}

Welding Research Council Bulletin 342, April 1989

\section{Significance}

The ferrite content of austenitic stainless steel welds must be tightly controlled. Welds that are too low in ferrite may exhibit hot cracking, while those that are too high may exhibit poor corrosion performance or cause stray magnetic fields in magnetic applications. While there are various techniques to measure the ferrite content in the final product, most engineers prefer to avoid problems from the very beginning, by choosing materials and procedures based on accurate predictions. The procedures and graph in this report have been adopted by many codes and standards, such as the ASME Boiler and Pressure Vessel Code. The American Welding Society selected a summary of this procedure for the 1989 McKay Helm Award, for "a significant advancement in the knowledge of stainless weld metals."

\begin{abstract}
A new diagram to predict the ferrite number $(\mathrm{FN})$ in stainless steel welds is proposed in this Bulletin. The diagram has a range from 0 to $100 \mathrm{FN}$ and more accurately predicts the ferrite content for welds having a FN less than 18 . The database contains over 950 welds and is included as an appendix to the report.
\end{abstract}

Publication of this report, WRC Bulletin No. 342 was sponsored by the Subcommittee on Welding Stainless Steel of the High Alloys Committee of the Welding Research Council. 


\title{
The accuracy of RASS temperature measurements
}

\author{
Peter T. May, Kenneth P. Moran, and Richard G. Strauch \\ NOAA Wave Propagation Laboratory
}

Journal of Applied Meteorology, 28(12): 1329-1335, December 1989

\section{Significance}

A Radio Acoustic Sounding System, or RASS, is a novel technique for remotely profiling temperature in the atmosphere. It uses clear-air Doppler radar technology to measure the speed of an upward propagating acoustic wave, which can be processed to yield a profile of the virtual temperature. Although the RASS concept was first proposed in the 1960s, successful implementations of the technology by various international research groups were delayed until the late 1970s and early 1980s because of hardware limitations. In the late 1980s, researchers at NOAA's Aeronomy Laboratory realized that this technique could be easily implemented on existing wind profiler radars that relied on scatter from clear-air turbulence to infer atmospheric winds. Subsequent work by NOAA's Environmental Technology Laboratory advanced the RASS technology to a point where it could be transitioned to the operational community. The cited paper represents a critical step towards the acceptance of RASS as a viable and important remote sensing tool. The authors performed a statistical study assessing the utility of the technique for meteorological studies by comparing RASS and radiosonde temperature measurements over extended periods during the summer and winter months of 1988 through 1989. Their study showed that the RASS technique yields temperature measurements comparable in accuracy with radiosonde observations, the current standard. Their conclusion led to the rapid adoption of the RASS technique by NOAA's Wind Profiler Demonstration Network. Tropospheric wind and temperature information can now be routinely retrieved using a large number of wind profiler/RASS installations across the country.

\begin{abstract}
Temperature measurements obtained using radiosondes and Radio Acoustic Sounding Systems (RASS) are compared to assess the utility of the RASS technique for meteorological studies. The agreement is generally excellent; rms, temperature, differences are about $1.0^{\circ} \mathrm{C}$ for comparisons during a variety of meteorological conditions. Observations taken under ideal circumstances indicate that a precision of about $0.2^{\circ} \mathrm{C}$ is achievable with the RASS technique. A processor being designed for RASS should allow routine temperature measurements approaching this precision.
\end{abstract}




\section{Automated Computer Time Service:}

\section{The NIST Automated Computer Time Service}

Judah Levine, Marc Weiss, Dickie D. Davis, David W. Allan, and D.B. Sullivan

NIST Time and Frequency Division

NIST Journal of Research, 94(5): 311-321, September-October, 1989

\section{Time synchronization using the Internet}

Judah Levine

NIST Time and Frequency Division

IEEE Transactions on Ultrasonic Ferromagnetics, 45: 450-460, March 1, 1998

\section{Significance}

These two papers describe unique NIST services initiated to meet demand for accurate time for computers, networks, and electronic information exchange. The explosion of the use of computers and networks in the 1980s and 1990s fundamentally changed the way information is exchanged for commerce, defense, science, and many other applications. Accurate and reliable timing became crucial for both the functioning of the networks and for accurately and traceably ordering electronic transactions.

NIST's Judah Levine was one of the first people to both recognize this need and develop practical and easy-to-use solutions. The first paper describes a system initiated in the 1980s to synchronize computers and other electronic devices to NIST time using an automated dialup system and free client software provided by NIST. This Automated Computer Time Service (ACTS) immediately became a crucial tool for information technology applications and grew rapidly with very little publicity on NIST's part.

Levine also anticipated early the impact of the Internet and initiated in the early 1990s a similar service using the Internet and free client software to automatically synchronize computer clocks and other networked devices to NIST time. This Internet Time Service (ITS) grew even more rapidly than the Internet itself. Today NIST processes more than 1 billion ITS requests for NIST time every day, with demand doubling about every 10 months since the service was introduced. The NIST software is free, but dozens of companies now provide their own versions of the software using ITS. The National Association of Securities Dealers (NASD) Order Audit Trail System requires that all of the electronic NASDAQ transactions be time-stamped to NIST time, involving more than 600,000 companies and tens of billions of dollars in transactions each day, and ACTS and ITS are the primary sources for complying with these regulations. NIST time provided through ITS and ACTS is by many orders of magnitude the most heavily and broadly used NIST service, and arguably the service with the largest direct economic impact. 


\begin{abstract}
1
The NIST Automated Computer Time Service (ACTS) is a telephone time service designed to provide computers with telephone access to time generated by the National Institute of Standards and Technology at accuracies approaching $1 \mathrm{~ms}$. Features of the service include automated estimation by the transmitter of the telephone-line delay, advanced alert for changes to and from daylight saving time, and advanced notice of insertion of leap seconds. The ASCII-character time code operates with most standard modems and computer systems. The system can be used to set computer clocks and simple hardware can also be developed to set non-computer clock systems.
\end{abstract}

\begin{abstract}
2
This paper discusses a new algorithm for synchronizing the clocks of networked computers using messages transmitted over the network itself. The design is based on a statistical model of the clock and the network, and uses this model to define the parameters of a frequency-lock loop which is used to discipline the local oscillator. The design was tested by synchronizing a standard workstation to a time server located $1200 \mathrm{~km}$ away; the time offset between the clock synchronized in this way and UTC is $2 \mathrm{~ms}$ rms. This analysis also can be used to design algorithms that provide lower accuracy at lower cost.
\end{abstract}




\section{Papers from the 1990s}

Improved technique for determining complex permittivity with the transmission/reflection method

James Baker-Jarvis, Eric J. Vanzura, and William Kissick, 1990

Observational constraints on the global atmospheric $\mathrm{CO}_{2}$ budget

Pieter Tans, Inez Fung, and Taro Takahashi, 1990

Alternating-field susceptometry and magnetic susceptibility of superconductors

Ron B. Goldfarb, Curt A. Thompson, and Mark Lelental, 1991

124

A model for the complex permittivity of water at frequencies below $1 \mathrm{THz}$

Hans J. Liebe, George A. Hufford, and Takeshi Manabe, 1991

An isentropic three-hourly data assimilation system using ACARS aircraft observations Stanley G. Benjamin, Keith A. Brewster, Renate Brümmer, Brian F. Jewett, Thomas W. Schlatter, Tracy L. Smith, and Peter A. Stamus, 1991

Characteristic impedance determination using propagation constant measurement Roger B. Marks and Dylan F. Williams, June 1991

Slowing down of the global accumulation of atmospheric methane during the 1980s L. Paul Steele, Edward J. Dlugokencky, Patricia M. Lang, Pieter P. Tans, Russell C. Martin, and Kenneth A. Masarie, 1992

A new method for measuring the strength and ductility of thin films

David T. Read and James W. Dally, 1993

Atmospheric lifetimes of long-lived halogenated species

Akkihebbal R. Ravishankara, Susan Solomon, Andrew A. Turnipseed, and Rachel F. Warren, 1993

Decrease in the growth rates of atmospheric chlorofluorocarbons 11 and 12 James W. Elkins, Thayne M. Thompson, James H. Butler, Bradley D. Hall, Thomas H. Swanson, Scott O. Cummings, Donald A. Fisher, and A.G. Raffo, 1993

Electron beam moiré

James W. Dally and David T. Read, 1993

Optical fiber geometry: Accurate measurement of cladding diameter Matt Young, Paul D. Hale, and Steven E. Mechels, 1993 
REFPROP Refrigerant Properties Database: Capabilities, limitations, and future directions Mark O. McLinden, John G. Gallagher, Marcia L. Huber, and Graham Morrison, 1993

Surface climate variations over the North Atlantic Ocean during winter: 1900-89

Clara Deser and Maurice L. Blackmon, 1993

Welding for cryogenic service

Thomas A. Siewert and Chris N. McCowan, 1993

Metrological accuracy of the electron pump

John M. Martinis and Michael Nahum, 1994

Response of the thermosphere and ionosphere to geomagnetic storms

Tim J. Fuller-Rowell, Mihail V. Codrescu, R.J. Moffett, and Shaun Quegan, 1994

A mechanism for the recurrence of wintertime midlatitude SST anomalies during winter Michael A. Alexander and Clara Deser, 1995

Analog-to-digital converters and their applications in radio receivers

Jeffery A. Wepman, 1995

Demonstration of a fundamental universal quantum logic gate

Chris Monroe, Dawn M. Meekhof, Brian E. King, Wayne M. Itano, and

David J. Wineland, 1995

The optimal growth of tropical sea surface temperature anomalies

Cécile Penland and Prashant D. Sardeshmukh, 1995

A "Schrödinger cat" superposition state of an atom

Chris Monroe, Dawn M. Meekhof, Brian E. King, and David J. Wineland, 1996

Solidification sensing using high-energy x-ray diffraction

Thomas A. Siewert, William P. Dubé, and Dale W. Fitting, 1996

X-ray detection using a superconducting transition-edge sensor microcalorimeter with electrothermal feedback

Kent D. Irwin, Gene C. Hilton, David A. Wollman, and John M. Martinis, 1996

Arctic environmental change of the last four centuries

Jonathan T. Overpeck, Konrad Hughen, Douglas Hardy, Raymond Bradley, Roslyn Case, Maryanne Douglas, Bruce Finney, Konrad Gajewski, Gordon Jacoby, Anne Jennings, Scott Lamoureux, Andrew Lasca, Glen MacDonald, Jay Moore, Michael Retelle, Sheldon Smith, Alex Wolfe, and Gregory Zielinski, 1997 
El Niño, La Niña, and the nonlinearity of their teleconnections

Martin P. Hoerling, Arun Kumar, and Min Zhong, 1997

Isochoric $(\mathrm{p}, \rho, \mathrm{T})$ measurements for five natural gas mixtures from

$\mathrm{T}=(225$ to 350$) \mathrm{K}$ at pressures to $35 \mathrm{MPa}$

Joseph W. Magee, William M. Haynes, and Michael J. Hiza, 1997

Mapping city lights with nighttime data from the DMSP Operational Linescan System Christopher D. Elvidge, Kimberly E. Baugh, Eric A. Kihn, Herbert W. Kroehl, and Ethan R. Davis, 1997

Electromagnetic theory of reverberation chambers

David A. Hill, 1998

2000 years of drought variability in the central United States

Connie A. Woodhouse and Jonathan T. Overpeck, 1998

A capacitance standard based on counting electrons

Mark W. Keller, Ali L. Eichenberger, John M. Martinis,

and Neil M. Zimmerman, 1999

For 100 years, notched bar impact testing standards have yielded widespread benefits for industry

Michael P. Manahan, Chris N. McCowan, Thomas A. Siewert, John M. Holt, Francis J. Marsh, and Earl A. Ruth, 1999

Objective estimation of perceived speech quality

Part I: Development of the measuring normalizing block technique

Part II: Evaluation of the measuring normalizing block technique

Stephen Voran, 1999 


\title{
Improved technique for determining complex permittivity with the transmission/reflection method
}

\author{
James Baker-Jarvis, Eric J. Vanzura, and William Kissick \\ NIST Electromagnetic Fields Division
}

IEEE Transactions on Microwave Theory and Techniques, 38(8): 1096-1101, 1990

\section{Significance}

This paper developed a new method for determining the permittivity from transmission-line scattering data. This new stable method for permittivity determination eliminated the instabilities that plagued the commonly used Nicolson-Ross method. The paper has been widely acclaimed as a breakthrough in transmission line permittivity measurements.

\begin{abstract}
The transmission/reflection method for complex permittivity and permeability determination is studied. The special case of permittivity measurement is examined in detail. New robust algorithms for permittivity determination that eliminate the ill-behaved nature of the commonly used procedures at frequencies corresponding to integer multiples of one-half wavelength in the sample are presented. An error analysis is presented which yields estimates of the error incurred due to the uncertainty in scattering parameters, length measurement, and reference plane position. In addition, new equations are derived for determining complex permittivity independent of reference plane position and sample length.
\end{abstract}




\title{
Observational constraints on the global atmospheric $\mathrm{CO}_{2}$ budget
}

\author{
Pieter Tans \\ NOAA Climate Monitoring and Diagnostics Laboratory \\ Inez Fung \\ National Atmospheric and Space Administration \\ Taro Takahashi \\ Columbia University
}

Science, 247: 1431-1438, 1990

\section{Significance}

This paper showed that North America was a potential sink for large amounts of $\mathrm{CO}_{2}$ that led to a completely new way of thinking about the global carbon cycle. It was used as an underpinning for the growing North American Carbon Cycle Research Program, the largest and most expensive global climate change research program on earth.

\begin{abstract}
Observed atmospheric concentrations of $\mathrm{CO}_{2}$ and data on partial pressures of $\mathrm{CO}_{2}$ in surface ocean waters are combined to identify globally significant sources and sinks of $\mathrm{CO}_{2}$. The atmospheric data are compared with boundary layer concentrations calculated with the transport field generated by a general circulations model (GCM) for specified source-sink distributions. In the model, the observed north-south atmospheric concentration gradient can be maintained only if the sinks for $\mathrm{CO}_{2}$ are greater in the Northern than in the Southern Hemisphere. The observed differences between the partial pressures of $\mathrm{CO}_{2}$ in the surface waters of the Northern Hemisphere and the atmosphere are too small for the oceans to be the major sink of fossil fuel $\mathrm{CO}_{2}$. Therefore, a large amount of the $\mathrm{CO}_{2}$ is apparently absorbed on the continents by terrestrial ecosystems.
\end{abstract}




\title{
Alternating-field susceptometry and magnetic susceptibility of superconductors
}

\author{
Ron B. Goldfarb and Curt A. Thompson \\ NIST Electromagnetic Fields Division \\ Mark Lelental \\ Eastman Kodak Company
}

NIST Internal Report 3977, October 1991; also published in Magnetic Susceptibility of Superconductors and Other Spin Systems, R.A. Hein, T.L. Francavilla, and D.H. Liebenberg, editors. New York: Plenum Press, 1991, pp. 49-80

\section{Significance}

This monograph is a thorough analysis of alternating-field susceptometry applied to superconductors. It has been cited widely as a comprehensive and authoritative guide to magnetic susceptibility measurements. Susceptometry has become a standard method for characterizing "weak-link" connected superconductors. The measurements and results have been replicated and used in laboratories throughout the world. Magnetic instrument manufacturers have reprinted it as "Tech Notes" for their customers.

\begin{abstract}
This review critically analyzes current practice in the design, calibration, sensitivity determination, and operation of alternating-field susceptometers, and examines applications in magnetic susceptibility measurements of superconductors. Critical parameters of the intrinsic and coupling components of granular superconductors may be deduced from magnetic susceptibility measurements. The onset of intrinsic diamagnetism corresponds to the initial decrease in electrical resistivity upon cooling, but the onset of intergranular coupling coincides with the temperature for zero resistivity. The lower critical field may be determined by the field at which the imaginary part of susceptibility increases from zero. Unusual features in the susceptibility of superconductor films, such as a magnetic moment that is independent of film thickness and the variation of susceptibility with angle, are related to demagnetization. Demagnetizing factors of superconductor cylinders are significantly different from those commonly tabulated for materials with small susceptibilities. Rules for the susceptibility of mixtures with specific demagnetizing factors are used to estimate the volume fraction of superconducting grains in sintered materials. Common misunderstandings of the Meissner effect, magnetic units, and formula conversions are discussed. There is a comprehensive summary of critical-state formulas for slabs and cylinders, including new equations for complex susceptibility in large alternating fields. Limitations on the use of the critical-state model for deducing critical current density are listed and the meaning of the imaginary part of susceptibility is considered.
\end{abstract}




\title{
A model for the complex permittivity of water at frequencies below 1 THz
}

\author{
Hans J. Liebe and George A. Hufford \\ NTIA Institute for Telecommunication Sciences \\ Takeshi Manabe \\ ATR Optical and Radio Communications Research Laboratories, Japan \\ International Journal of Infrared and Millimeter Waves, 12(7): 659-675, 1991
}

\section{Significance}

The dielectric properties (i.e., complex permittivity) of liquid water at atmospheric temperatures play a key role in computations of atmospheric propagation and remote sensing effects caused by raindrops and by droplets suspended in fog or clouds. Also, systems that operate in the nearmillimeter-wave (NMW, $0.1 \mathrm{THz}$ to $1 \mathrm{THz}$ ) frequency range experience much less loss than those utilizing infrared/optical frequencies in inclement weather and it is useful to have a reliable model to compute the advantage. This model substantially improved on the existing standard model (Ray, 1972) by utilizing both a considerable body of new experimental evidence and a simplified and more accurate double Debye (i.e., relaxation) term model formulation for the dielectric properties. The improved model, although developed primarily for use with the ITS millimeter wave propagation model (MPM, see Liebe, 1989, this volume), is widely recognized and has been embraced by a wide range of atmospheric science researchers and communications engineers.

\begin{abstract}
Experimental permittivity data of liquid water, compiled from the open literature, were selectively applied to support a modeling strategy. Frequencies up to $1 \mathrm{THz}$ and atmospheric temperatures are covered with an expression made up by two relaxation (Debye) terms. The double-Debye model reduces to one term when the high frequency limit is set at $100 \mathrm{GHz}$, and the model can be extended to $30 \mathrm{THz}$ by adding two resonance (Lorentzian) terms. The scheme was carried out by employing nonlinear least-squares fitting routines to data we considered reliable.
\end{abstract}




\title{
An isentropic three-hourly data assimilation system using ACARS aircraft observations
}

Stanley G. Benjamin

NOAA Forecast Systems Laboratory

Keith A. Brewster

University of Oklahoma, Norman, $O K$

Renate Brümmer

Deutsche Forschungsanstalt für Luft- und

Raumfahrt, Cologne, Germany

\author{
Brian F. Jewett \\ University of Illinois, Champaign, IL \\ Thomas W. Schlatter \\ NOAA Forecast Systems Laboratory \\ Tracy L. Smith and Peter A. Stamus \\ Cooperative Institute for Research \\ in the Atmosphere
}

Monthly Weather Review, 119: 888-906, 1991

\section{Significance}

The Mesoscale Analysis and Prediction System (MAPS) described in this paper made it possible to produce maps of surface and upper air weather conditions and, from them, generate short-term forecasts at three-hour intervals. Prior to this time, computer forecasts were started only at 0000 and $1200 \mathrm{GMT}$, following weather balloon launches. Observations made at other times were largely ignored. The frequent updating of analyses and forecasts led to improved guidance for commercial flight planning and local weather services. Also, the use of isentropic coordinates (surfaces of constant potential temperature) led to better predictions near the tropopause and in the vicinity of warm and cold fronts. MAPS, developed with the cooperation of the National Meteorological Center (NMC), was the forerunner of the Rapid Update Cycle (RUC), implemented operationally in 1994 and still running - today on an hourly cycle. MAPS and the RUC are examples of effective technology transfer. Forecasters at National Weather Service Offices use the RUC for short-range guidance; the Storm Prediction Center uses it routinely for severe weather watches; the Aviation Weather Center uses it for aviation weather advisories; the FAA uses it for estimating weather constraints on air traffic flow.

\begin{abstract}
A 3-h intermittent data assimilation system (MAPS) configured in isentropic coordinates was developed and implemented in real-time operation. The major components of this system are data ingest, objective quality control of the observations, objective analysis of the observations on a grid, and a primitive equation forecast model, all using isentropic coordinates. The primary data sources for this system are automated aircraft reports, wind profilers, surface observations, and rawinsonde balloons. Three-hour forecasts from MAPS incorporating the aircraft reports improve on 12-h MAPS forecasts valid at the same time for all levels and variables, and also improve on 12-h NMC forecasts of upper level winds. This result is due to frequent updating, here demonstrated for the first time, the quality and volume of the aircraft data, and the effectiveness of the isentropic data assimilation used.
\end{abstract}




\title{
Characteristic impedance determination using propagation constant measurement
}

\author{
Roger B. Marks and Dylan F. Williams \\ NIST Electromagnetic Fields Division \\ IEEE Microwave and Guided Wave Letters, 1(6): 141-143, June 1991
}

\section{Significance}

This paper developed the first accurate and straightforward method of measuring the frequency dependent characteristic impedance of on-wafer transmission lines. The method enables the onwafer TRL calibration, which, thanks to this work, has become the reference calibration to which all others are compared.

\begin{abstract}
A method is demonstrated whereby the characteristic impedance of transmission lines may be easily determined from a measurement of the propagation constant. The method is based on a rigorous analysis using realistic approximations to account for the effects of imperfect conductors. Numerical studies indicate that high accuracy is possible, and experiments using coplanar waveguide demonstrate the advantage of the method in the interpretation of $S$ parameters.
\end{abstract}




\title{
Slowing down of the global accumulation of atmospheric methane during the 1980s
}

\author{
L. Paul Steele \\ NOAA Climate Monitoring and Diagnostics Laboratory/CIRES/CSIRO Australia \\ Edward J. Dlugokencky \\ NOAA Climate Monitoring and Diagnostics Laboratory/CIRES \\ Patricia M. Lang and Pieter P. Tans \\ NOAA Climate Monitoring and Diagnostics Laboratory \\ Russell C. Martin and Kenneth A. Masarie \\ NOAA Climate Monitoring and Diagnostics Laboratory/CIRES
}

Nature, 358: 313-316, 1992

\section{Significance}

This was the first study to show that the rate of increase of methane, which had been steadily increasing since the beginning of the industrial revolution, was unexpectedly decreasing. This discovery was made through study of the long-term atmospheric trace gas concentration data collected on a global scale by the decades-old CMDL cooperative flask sampling program. Methane is second only to carbon dioxide in its anthropogenic influence on climate, contributing about $20 \%$ of the total increase in radiative forcing by long-lived greenhouse gases. It is also important in determining the cleansing capacity of the troposphere. The atmospheric burden of methane increased by a factor of 2.5 from pre-industrial times to the early $1980 \mathrm{~s}$. Until this paper, most scientists thought that the rate of increase of atmospheric methane would continue to grow as global population increased. This work showed that the growth of population and methane emissions had unexpectedly become decoupled. This discovery has resulted in downward revisions to estimates of the future rate of increase in atmospheric methane used to predict climate change, possibly the greatest challenge to society in this century.

\begin{abstract}
Measurements of methane in modern air and in air trapped in ice cores have shown convincingly that the abundance of atmospheric methane has been rising since the Industrial Revolution. This is a matter of concern because of the important role of methane in determining the radiative balance and chemical composition of the atmosphere. The causes of this increase have not been identified unambiguously because of uncertainties in our understanding of the global budget of atmospheric methane and in how it is changing with time. Here we report on measurements of atmospheric methane from an extensive global network of flask sampling sites, which reveal that, although methane continues to accumulate in the atmosphere, there has been a substantial slowing of the global accumulation rate between 1983 and 1990. If this deceleration continues steadily, global methane concentrations will reach a maximum around the year 2006. Our results hint that changes in methane emissions in the latitude band 30-90-degrees $\mathrm{N}$ may be of particular significance to this trend.
\end{abstract}




\title{
A new method for measuring the strength and ductility of thin films
}

\author{
David T. Read \\ NIST Materials Reliability Division \\ James W. Dally \\ Mechanical Engineering Department, University of Maryland
}

Journal of Materials Research 8: 1542-1549, July 1993

\section{Significance}

This paper was our first publication on mechanical properties of thin films. It marks the beginning of the effort at NIST to develop mechanical property measurements for thin films used in microelectronic devices. This paper was the beginning of our attempt to push the size of mechanical test specimens down toward the actual size of films used in electronic devices.

\begin{abstract}
A new method of measuring the mechanical strength of thin films is described. We prepare miniature arrays of four tensile specimens, each $0.25 \mathrm{~mm}$ wide, $1 \mathrm{~mm}$ long, and $2.2 \mu \mathrm{m}$ thick, using deposition, patterning, and etching processes common to the semiconductor industry. Each array of four specimens is carried on and protected by a rectangular silicon frame. Thirty-six such specimens are produced on a single wafer. After a specimen frame is mounted, its vertical sides are severed without damaging the specimens. The load is applied by micrometers through a special tension spring. Tensile properties of a $2.2 \mu \mathrm{m}$ thick Ti-Al-Ti film were determined.
\end{abstract}




\title{
Atmospheric lifetimes of long-lived halogenated species
}

\author{
Akkihebbal R. Ravishankara, Susan Solomon, Andrew A. Turnipseed, and Rachel F. Warren \\ NOAA Aeronomy Laboratory
}

Science, 259: 194-199, 1993

\section{Significance}

Perfluorocarbons (PFCs) are a class of compounds that were being considered as replacements for the now-banned ozone-destroying chlorofluorocarbons (CFCs) in industrial applications such as fire extinguishing, foam blowing, and refrigeration. PFCs have essentially zero ozonedestroying capacity and can be considered "ozone-friendly." However, as pointed out by this paper, it is important also to consider the possible role of PFCs as greenhouse gases.

Ravishankara et al. determined the atmospheric lifetime of six of these gases, a crucial parameter used in the calculation of the Global Warming Potential (GWP) index used by policymakers to gauge each compound's global climate impact. The researchers found that these chemical species had atmospheric residence times of 2,000 to 50,000 years (many would live longer than the past human-civilization time span!) and were up to 12,000 times more potent greenhouse gases than carbon dioxide. The implications for policymakers of these newly recognized "immortal" greenhouse gases were threefold: (1) emissions of PFCs would accumulate in the atmosphere for tens of centuries; (2) the Global Warming Potentials for these compounds would be much larger than the CFCs they would replace; and (3) it would take the atmosphere an extremely long time to recover from any inputs of these compounds. This paper delivered sound science, in a timely manner, in response to a great need in the national and international community, and opened up research in this area in the process. By averting the use of what would have been very unsuitable substitutes for the CFCs, the paper spared industry a great expense while simultaneously protecting the atmosphere from additional burdens of potent greenhouse gases.

\begin{abstract}
The atmospheric lifetimes of the fluorinated gases $\mathrm{CF}_{4}, \mathrm{C}_{2} \mathrm{~F}_{6}, \mathrm{c}-\mathrm{C}_{4} \mathrm{~F}_{8},\left(\mathrm{CF}_{3}\right)_{2} \mathrm{c}-\mathrm{C}_{4} \mathrm{~F}_{6}, \mathrm{C}_{5} \mathrm{~F}_{12}$, $\mathrm{C}_{6} \mathrm{~F}_{14}, \mathrm{C}_{2} \mathrm{~F}_{6} \mathrm{Cl}, \mathrm{C}_{2} \mathrm{~F}_{4} \mathrm{C}_{2}, \mathrm{CF}_{3} \mathrm{Cl}$, and $\mathrm{SF}_{6}$ are of concern because of the effects that these long-lived compounds acting as greenhouse gases can have on global climate. The possible atmospheric loss processes of these gases were assessed by determining the rate coefficients for the reactions of these gases with $\mathrm{O}\left({ }^{1} D\right), \mathrm{H}$, and $\mathrm{OH}$ and the absorption cross sections at 121.6 nanometers in the laboratory and using these data as input to a two-dimensional atmospheric model. The lifetimes of all the studied perfluoro compounds are $>2000$ years, and those of $\mathrm{CF}_{3} \mathrm{Cl}_{,} \mathrm{CF}_{3} \mathrm{CF}_{2} \mathrm{Cl}$, and $\mathrm{CF}_{2} \mathrm{ClCF}_{2} \mathrm{Cl}$ are $>300$ years. If released into the atmosphere, these molecules will accumulate and their effects will persist for centuries or millennia.
\end{abstract}




\title{
Decrease in the growth rates of atmospheric chlorofluorocarbons 11 and 12
}

James W. Elkins, Thayne M. Thompson, James H. Butler, and Bradley D. Hall

NOAA Climate Monitoring and Diagnostics Laboratory

Thomas H. Swanson and Scott O. Cummings

NOAA Climate Monitoring and Diagnostics Laboratory/CIRES

Donald A. Fisher and A.G. Raffo

Dupont, Inc.

Nature, 364: 780-783, August 26, 1993

\section{Significance}

Atmospheric measurements of CFC-11 and CFC-12 were initiated by CMDL in 1977 at four NOAA baseline stations and at four cooperative sites stretching from near the North Pole to the South Pole. In 1985 the Antarctic Ozone Hole was discovered and the cause subsequently found to be a chemical reaction fueled by CFCs and related halogen compounds that were subsequently controlled by the Montreal Protocol. This paper shows that the steady growth rate of CFC-11 and CFC-12 in the atmosphere, first established by the CMDL measurements, were beginning to decrease. This landmark paper provided proof that international efforts to limit emissions of ozone depleting substances were effective and provided encouragement that the stratospheric ozone hole phenomenon could be controlled. The value of long-term monitoring of atmospheric constituents, even when the role they play in atmospheric chemistry is not clearly known, is superbly illustrated by the data presented in this paper.

\begin{abstract}
The discovery of the Antarctic ozone hole in 1985 led to international efforts to reduce emissions of ozone-destroying chlorofluorocarbons. These efforts culminated in the Montreal Protocol and its subsequent amendments, which called for the elimination of CFC production by 1996 . Here we focus on $\mathrm{CFC}-11(\mathrm{CCl} 3 \mathrm{~F})$ and $\mathrm{CFC}-12(\mathrm{CCl} 2 \mathrm{~F} 2)$, which are used for refrigeration, air conditioning and the production of aerosols and foams, and which together make up about half of the total abundance of stratospheric organic chlorine. We report on a significant recent decrease in the atmospheric growth rates of these two species, based on measurements spanning the past 15 years and latitudes ranging from $83^{\circ} \mathrm{N}$ to $90^{\circ} \mathrm{S}$. This is consistent with CFC-producers' own estimates of reduced emissions. If the atmospheric growth rates of these two species continue to slow in line with predicted changes in industrial emission, global atmospheric mixing ratios will reach a maximum before the turn of the century, and then begin to decline.
\end{abstract}




\title{
Electron beam moiré
}

James W. Dally

Mechanical Engineering Department, University of Maryland

David T. Read

NIST Materials Reliability Division

Experimental Mechanics, 33: 270-277, December 1993

\section{Significance}

This paper was one of the first to go to size scales smaller than can be handled with optical microscopy, to apply scanning electron microscopy as a tool in experimental mechanics. The SEM was used both to create a grating on the specimen, and to image changes in the grating as the specimen deformed.

\begin{abstract}
A method of writing very high frequency line and dot patterns, in excess of 10,000 lines $/ \mathrm{mm}$, is described. This method uses a very small diameter, 10 to $20 \mathrm{~nm}$, beam of electrons to sensitize a 100-nm thick layer of electron resist. The line and dot patterns are produced by etching the sensitized resist. Moiré fringe patterns occur when the line arrays are observed in the scanning electron microscope. Moiré fringes with excellent contrast have been produced at magnifications as high as 1900x. This capability permits e-beam moiré to be employed in micromechanics. Examples of line arrays, dot arrays and moiré fringe patterns on a brass disk and on a tensile specimen fabricated from glass-fiber-reinforced plastic are demonstrated to introduce the possibilities for micromechanics applications.
\end{abstract}




\title{
Optical fiber geometry: Accurate measurement of cladding diameter
}

\author{
Matt Young, Paul D. Hale, and Steven E. Mechels \\ NIST Electromagnetic Technology Division
}

NIST Journal of Research, 98: 203-216, 1993

\section{Significance}

This paper is the final summary of work on standards for the measurement of the diameter of optical fiber and the development of NIST Standard Reference Material 2520. This was one of NIST's most successful programs in support of the optical communications industry. It allowed fiber manufacturers to reduce their uncertainties and, for a while, gave American manufacturers a competitive advantage over manufacturers from other countries.

\begin{abstract}
We have developed three instruments for accurate measurement of optical fiber cladding diameter: a contact micrometer, a scanning confocal microscope, and a white-light interference microscope. Each instrument has an estimated uncertainty (3 standard deviations) of $50 \mathrm{~nm}$ or less, but the confocal microscope may display a $20 \mathrm{~nm}$ systematic error as well. The micrometer is used to generate standard Reference Materials that are commercially available.
\end{abstract}




\title{
REFPROP Refrigerant Properties Database: Capabilities, limitations, and future directions
}

\author{
Mark O. McLinden, John G. Gallagher, Marcia L. Huber, and Graham Morrison \\ NIST Thermophysics Division \\ Proceedings of the ASHRAE/NIST Refrigerants Conference, Gaithersburg, MD, 1993
}

\section{Significance}

A potential environmental crisis was identified in the 1970s with the observation that man-made chemicals (chlorofluorocarbons or CFCs) used in refrigerators and air conditioners contribute to the destruction of the earth's stratospheric ozone layer. The environmental issues and international agreements on the use of refrigeration fluids resulted in a very large effort at NIST to consider and study alternative refrigeration working fluids. The REFPROP database, described in this paper, was developed to meet the needs of the refrigeration industry in their evaluation of alternative refrigerants. The computer program, allowing calculations of properties at arbitrary temperatures and pressures, quickly became the de facto standard in the industry, permitting a consensus on the numbers to use in the design and evaluation of alternative refrigerant systems. The NIST work on refrigerating fluids was able not only to help reduce the impact on the ozone layer, but also to generate cooling system designs that use substantially less energy than the older technologies. The REFPROP database described here remains a standard, and its capabilities have been substantially extended in the years since this paper appeared. The models which evolved from the work described here have been incorporated in an ISO standard that was adopted in 2004.

\begin{abstract}
The REFPROP computer database has been developed to meet the needs of the refrigeration industry in their evaluation of alternative refrigerants. In this paper we highlight the capabilities and discuss the limitations of the database through examples of several representative pure refrigerants and refrigerant blends. The database employs the Carnahan-Starling-Desantis (CSD), modified Bennedict-Webb-Rubin (MBWR), and Haar-Gallagher equations of state for thermodynamic properties, and an extended corresponding states (ECS) model for thermodynamic and transport properties; the most appropriate model for specific applications is recommended.
\end{abstract}




\title{
Surface climate variations over the North Atlantic Ocean during winter: 1900-89
}

\author{
Clara Deser and Maurice L. Blackmon \\ NOAA/CIRES Climate Diagnostics Center
}

Journal of Climate, 6: 1743-1753, September 1993

\section{Significance}

This well-cited paper revealed clear evidence for decadal variability in the North Atlantic Ocean, overlying atmosphere and sea ice. One intriguing result, among many, was that ice anomalies in the Labrador Sea lead SST anomalies in the North Atlantic by 2 years. Overall, the analyses suggested that ice-ocean-atmosphere interaction could result in low-frequency ( $>10$ years) variability in a number of ways and strongly motivated the research community to model and explain this variability.

\begin{abstract}
The low-frequency variability of the surface climate over the North Atlantic during winter is described, using 90 years of weather observations from the Comprehensive Ocean-Atmosphere Data Set. Results are based on empirical orthogonal function analysis of four components of the climate system: sea surface temperature (SST), air temperature, wind, and sea level pressure. An important mode of variability of the wintertime surface climate over the North Atlantic during this century is characterized by a dipole pattern in SSTs and surface air temperatures, with anomalies of one sign east of Newfoundland, and anomalies of the opposite polarity off the southeast coast of the United States. Wind fluctuations occur locally over the regions of large surface temperature anomalies, with stronger-than-normal winds overlying cooler-than-normal SSTs. This mode exhibits variability on quasi-decadal and biennial time scales. The decadal fluctuations are irregular in length, averaging $\sim 9$ years before 1945 and $\sim 12$ years afterward. There does not appear to be any difference between the wind-SST relationships on the different time scales. The decadal fluctuations in SSTs east of Newfoundland are closely linked to decadal variations in sea ice in the Labrador Sea, with periods of greater than normal sea ice extent preceding by $\sim 2$ years periods of colder-than-normal SSTs east of Newfoundland.

Another dominant mode of variability is associated with the global surface warming trend during the 1920s and 1930s. The patterns of SST and air temperature change between 1900-29 and 1939-68 indicate that the warming was concentrated along the Gulf Stream east of Cape Hatteras. Warming also occurred over the Greenland Sea and the eastern subtropical Atlantic. The warming trend was accompanied by a decrease in the strength of the basin-scale atmospheric circulation (negative phase of the North Atlantic Oscillation). In marked contrast to the dipole pattern, the wind changes occurred downstream of the largest SST anomalies; hence, the gradual surface warming along the Gulf Stream may have been a result of altered ocean currents rather than local wind forcing.
\end{abstract}




\title{
Welding for cryogenic service
}

\author{
Thomas A. Siewert and Chris N. McCowan \\ NIST Materials Reliability Division
}

Chapter in ASM Handbook: Volume 6 (Welding), Materials Park, Ohio: ASM International, 1016-1019, 1993

\section{Significance}

This book chapter summarizes about 20 years of research at NIST on the effect of welding on the behavior of fabricated structures at cryogenic temperatures. The behavior of the welds is similar to that of the plate material in many ways, yet there are some important differences and some special considerations. Therefore, this data complements the other reports on plates and sheets, and serves to guide fabricators and welding engineers on the key joining issues. This report covers the factors that determine the strength and toughness of the welds at temperatures down to $4 \mathrm{~K}$, and includes tables to illustrate the trends. It includes some comments on other important properties such as fatigue strength, and coefficient of thermal expansion (which becomes particularly important when dissimilar filler metals are chosen). It also discusses the powerful effect on toughness of inclusions (predominately oxides) in the weld pool. This book chapter was one of our last publications in this area and so serves as a summary of our final understanding of the most important factors.

\begin{abstract}
Cryogenic temperatures cause many structural alloys to become brittle, which is an unacceptable condition in most structural applications. Therefore, structures built for service at low temperatures are typically made from alloys that maintain some ductility at the service temperatures. Cryogenic alloys include 9Ni steels, austenitic stainless steels, manganese stainless steels, maraging steels, titanium, aluminum, and nickel alloys. The choice of weld-metal alloy may depend solely on the strength of the alloy at a given temperature or on a combination of strength, toughness, fatigue resistance, thermal conductivity, magnetic permeability, and other considerations.

Wrought or cast alloy structural members are usually joined using filler materials with compositions that are similar, but not identical, to the parent material. The weld composition is optimized to compensate for the inherent differences in the properties of weld and parent material that are due to grain structure, inclusion content, and cooling rate differences. The goal is to match, as closely as possible, the mechanical and physical properties of the weld and parent material. When nonmatching weld compositions are most appropriate, differences between the welds and parent material in terms of thermal contraction, corrosion, and other factors must be considered. This article discusses these differences and explains how they affect the choice of the weld filler metal.
\end{abstract}




\title{
Metrological accuracy of the electron pump
}

John M. Martinis and Michael Nahum

NIST Electromagnetic Technology Division

Physical Review Letters, 72: 904-907, 1994

\section{Significance}

The charge of an electron is so small that the current in an ordinary flashlight bulb amounts to more than $10,000,000,000,000,000,000$ electrons passing through every second. How can you get a hold of just one? That requires a tricky bit of engineering, including a circuit so small it must be fabricated using an electron microscope and temperatures so cold that absolute zero is only a fraction of a degree colder. However, as John Martinis and Michael Nahum demonstrated in 1994, with such apparatus, you can not only manipulate electrons one by one, but count them with an accuracy of 0.5 parts per million. This pioneering work opened the possibility of making new fundamental standards for both electrical current and capacitance.

\begin{abstract}
We have operated a five-junction electron pump with an error for transferring electrons of approximately 0.5 part per $10^{6}$. The error predicted from existing theory is several orders of magnitude smaller, thus implying that our present understanding of the Coulomb blockade is incomplete. We conjecture that the errors arise from photon-assisted tunneling, where the photon energy is supplied by noise from the environment.
\end{abstract}




\title{
Response of the thermosphere and ionosphere to geomagnetic storms
}

\author{
Tim J. Fuller-Rowell and Mihail V. Codrescu \\ NOAA Space Environment Laboratory/CIRES \\ R.J. Moffett and Shaun Quegan \\ University of Sheffield
}

Journal of Geophysical Research, 99: 3893-3914, 1994

\section{Significance}

The ionosphere during geomagnetic storms exhibits complicated and apparently inexplicable behavior often described as chaotic. This paper recognized the interplay between the instant a magnetic storm begins and the subsequent behavior of the ionosphere above an arbitrary location. Through the use of a sophisticated model, the work demonstrated that the apparent chaotic behavior could be explained and predicted in detail using a minimum of physical assumptions. In doing so, a complex physical problem, often thought intractable, was resolved.

This paper described the balance of physical processes responsible for the ionospheric response to geomagnetic storms. In particular, Fuller-Rowell et al. were able to show the dependence on Universal Time, and explain the origin of the local time modulation in the positive and negative phases. Previously the storm-time response was regarded as chaotic, with positive and negative phases appearing randomly. The paper provides a framework for analysis of the observed ionospheric response to geomagnetic storms. In addition to separating local time from $\mathrm{UT} /$ longitude dependence, the paper provided an explanation of that part of the response that is consistent from one storm to the next. The physical insight enabled an empirical storm-time prediction model to be developed for the International Reference Ionosphere, something that was not possible before. While a relatively recent publication, this paper has been cited 120 times since publication with 86 "significances" since 1998 attesting to the growing recognition of its importance.

\begin{abstract}
Four numerical simulations have been performed, at equinox, using a coupled thermosphereionosphere model to illustrate the response of the upper atmosphere to geomagnetic storms. The storms are characterized by an increase in magnetospheric energy input at high latitude for a 12hour period; each storm commences at a different universal time (UT). The initial response at high latitude is that Joule heating raises the temperature of the upper atmosphere and ion drag drives high-velocity neutral winds. The heat source drives a global wind surge, from both polar regions, which propagates to low latitudes and into the opposite hemisphere. The surge has the character of a large-scale gravity wave with a phase speed of about $600 \mathrm{~m} \mathrm{~s}^{-1}$. Behind the surge a global circulation of magnitude $100 \mathrm{~m} \mathrm{~s}^{-1}$ is established at middle latitudes, indicating that the wave and the onset of global circulation are manifestations of the same phenomena. A dominant feature of the response is the penetration of the surge into the opposite hemisphere where it
\end{abstract}


drives poleward winds for a few hours. The global wind surge has a preference for the night sector and for the longitude of the magnetic pole and therefore depends on the UT start time of the storm. A second phase of the meridional circulation develops after the wave interaction but is also restricted, in this case by the buildup of zonal winds via the Coriolis interaction. Conservation of angular momentum may limit the buildup of zonal wind in extreme cases. The divergent wind field drives upwelling and composition change on both height and pressure surfaces. The composition bulge responds to both the background and the storm-induced horizontal winds; it does not simply rotate with Earth. During the storm the disturbance wind modulates the location of the bulge; during the recovery the background winds induce a diurnal variation in its position. Equatorward winds in sunlight produce positive ionospheric changes during the main driving phase of the storm. Negative ionospheric phases are caused by increases of molecular nitrogen in regions of sunlight, the strength of which depends on longitude and the local time of the sector during storm input. Regions of positive phase in the ionosphere persist in the recovery period due to decreases in mean molecular mass in regions of previous downwelling. Ion density changes, expressed as a ratio of disturbed to quiet values, exhibit a diurnal variation that is driven by the location of the composition bulge; this variation explains the ac component of the local time variation of the observed storm phase. 


\title{
A mechanism for the recurrence of wintertime midlatitude SST anomalies during winter
}

\author{
Michael A. Alexander and Clara Deser \\ NOAA Climate Monitoring and Diagnostics Laboratory/CIRES
}

Journal of Physical Oceanography, 25: 122-137, 1995

\section{Significance}

This paper was the first to clearly demonstrate that temperature anomalies that form at the surface and extend downward into the deep ocean mixed layer in winter, could remain intact in the seasonal thermocline during the following summer, and then reappear at the surface when the mixed layer again deepened in the following fall/winter. This process, termed the "reemergence mechanism," was confirmed using both subsurface temperature data and mixed layer model simulations. The reemergence mechanism provides an avenue to predict sea surface temperature anomalies and their potential impact on the atmospheric circulation.

\begin{abstract}
In the early 1970s, Namias and Born speculated that ocean temperature anomalies created over the deep mixed layer in winter could be preserved in the summer thermocline and reappear at the surface in the following fall or winter. This hypothesis is examined using upper-ocean temperature observations and simulations with a mixed layer model. The data were collected at six ocean weather stations in the North Atlantic and North Pacific. Concurrent and lead-lag correlations are used to investigate temperature variations associated with the seasonal cycle in both the observations and the model simulations.

Concurrent correlations between the surface and subsurface temperature anomalies in both the data and the model indicate that the penetration of temperature anomalies into the ocean is closely tied to the seasonal cycle in mixed layer depth: high correlations extend to relatively deep (shallow) depths in winter (summer). Lead-lag correlations in both the data and the model, at some of the stations, indicate that temperature anomalies beneath the mixed layer in summer are associated with the temperature anomalies in the mixed layer in the previous winter/spring and following fall/winter but are unrelated or weakly opposed to the temperature anomalies in the mixed layer in summer. These results suggest that vertical mixing processes allow ocean temperature anomalies created over a deep mixed layer in winter to be preserved below the surface in summer and reappear at the surface in the following fall, confirming the Namias-Born hypothesis.
\end{abstract}




\title{
Analog-to-digital converters and their applications in radio receivers
}

\author{
Jeffery A. Wepman \\ NTIA Institute for Telecommunication Sciences
}

IEEE Communications Magazine, 39-45, May 1995

\section{Significance}

This article provides a foundation for understanding analog-to-digital converters in software defined radio (SDR) receiver applications. It is based on pioneering research on SDR's conducted at ITS. An SDR is a radio consisting of: (a) a receiver where the received signal is digitized and then processed using software-programmable digital signal processing techniques, and/or (b) a transmitter where the modulated signal to be transmitted is generated as a digital signal using software-programmable digital signal processing techniques; the digital signal is then converted to an analog signal for transmission. The software programmability of an SDR allows easy changes of the radio's fundamental characteristics such as modulation type, operating frequency, and bandwidth.

The two essential components in an SDR receiver are analog-to-digital converters and digital signal processing hardware. This article offers an extremely clear, comprehensive, and yet concise discussion of both the theoretical and practical aspects of analog-to-digital converters for radio receiver applications, including sampling methods, effects of quantization noise, and important specifications such as signal-to-noise ratio, spurious-free dynamic range, and noise power ratio. Over the past ten years, development of SDR's has increased dramatically and continues to gain momentum. SDR technology has already been used in cellular and PCS base stations as well as fixed-station military radios. Future proliferation of SDR's is anticipated as the migration from traditional radio to SDR technology in handheld devices such as cellular telephones and portable public safety and military radios is realized.

\begin{abstract}
Rapid advances in hardware development of analog-to-digital converters (ADCs) have paved the way for development of radio receivers using digitization at the IF, and in some cases, at the RF. The constraints placed on these receivers due to hardware limitations of these devices are discussed and some examples of high-speed, state-of-the-art ADCs are given.
\end{abstract}




\title{
Demonstration of a fundamental universal quantum logic gate
}

\author{
Chris Monroe, Dawn M. Meekhof, Brian E. King, Wayne M. Itano, and David J. Wineland \\ NIST Time and Frequency Division
}

Physical Review Letters, 75: 4714-4717, 1995

\section{Significance}

This paper reported the world's first experimental demonstration that the quantum mechanical properties of atoms could be used to perform simple logic operations, a crucial first step toward building a quantum computer. Quantum computing is one of the most exciting areas of research today, with the potential to transform information technology and our understanding of the microscopic world.

This work built on the mastery of Dave Wineland's group in using laser cooling and trapping and exquisite manipulation of individual ions (charged atoms) first demonstrated in Wineland et al., 1978 (this volume). Quantum computing seeks to use the unique properties of quantum mechanics to develop systems based on a small number of quantum bits (qubits) such as ions, atoms, or nanoscale devices that will be able to perform certain kinds of very important calculations many orders of magnitude faster than today's best electronic (classical) computers. Quantum information is one of the most exciting and demanding fields in physics and information sciences today, attracting many of the world's top scientists and mathematicians. Essentially, it promises to make possible calculations that are now impossible and to introduce a type of computation that is more different from modern electronic computers than those computers are from the abacus. Wineland helped launch the field of experimental quantum computing and continues to be a world leader. This paper reports the demonstration of a "controlled-NOT" quantum logic gate, which in conjunction with simple single-bit operations, forms a universal quantum logic gate for quantum computation.

\footnotetext{
Abstract

We demonstrate the operation of a two-bit "controlled-NOT" quantum logic gate, which, in conjunction with simple single-bit operations, forms a universal quantum logic gate for quantum computation. The two quantum bits are stored in the internal and external degrees of freedom of a single trapped atom, which is first laser cooled to the zero-point energy. Decoherence effects are identified for the operation, and the possibility of extending the system to more qubits appears promising.
} 


\title{
The optimal growth of tropical sea surface temperature anomalies
}

\author{
Cécile Penland and Prashant D. Sardeshmukh \\ NOAA Climate Monitoring and Diagnostics Laboratory/CIRES
}

Journal of Climate, 8: 1999-2024, 1995

\section{Significance}

Prior to this paper, ENSO events were thought to arise from the instability of coupled air-sea interactions in the tropical Indo-Pacific basin. This paper presented strong evidence that the coupled interactions were in fact stable and governed by linear, randomly perturbed dynamics. An El Niño or La Niña event was argued to arise from the random significance of an "optimal" initial structure that would then amplify linearly into a full-blown event before fading away. This view of ENSO has gradually become widely accepted, and has proven invaluable in the development of models of ENSO prediction.

\begin{abstract}
It is argued from SST observations for the period 1950-90 that the tropical Indo-Pacific oceanatmosphere system may be described as a stable linear dynamical system driven by spatially coherent Gaussian white noise. Evidence is presented that the predictable component of SST anomaly growth is associated with the constructive interference of several damped normal modes after an optimal initial structure is set up by the white noise forcing. In particular, El NiñoSouthern Oscillation (ENSO) growth is associated with an interplay of at least three damped normal modes, with periods longer than two years and decay times of 4 to 8 months, rather than the manifestation of a single unstable mode whose growth is arrested by nonlinearities. Interestingly, the relevant modes are not the three least damped modes of the system. Rather, mode selection, and the establishment of the optimal initial structure from which growth occurs, are controlled by the stochastic forcing. Experiments conducted with an empirical stochasticdynamical model show that stochastic forcing not only adds energy to the system, but also plays a role in setting up the optimal structure.

It is shown that growth from modal interference can occur for as long as 18 months, which within the confines of this model defines a predictability limit for growth events. Growth associated with the stochastic forcing is also possible, but is unpredictable. The timescale on which the predictable and unpredictable components of SST growth become comparable to each other gives a more conservative predictability limit of 15 months.

The above scenario is based on empirical evidence obtained from SST anomalies alone. From the results of several tests based on statistical properties of linear and nonlinear dynamical systems, one may conclude that much of the ENSO cycle in nature is dominated by stable, forced dynamics.
\end{abstract}




\title{
A "Schrödinger cat" superposition state of an atom
}

\author{
Chris Monroe, Dawn M. Meekhof, Brian E. King, and David J. Wineland \\ NIST Time and Frequency Division
}

Science, 272: 1131-1136, May 24, 1996

\section{Significance}

Dave Wineland's ability to exquisitely control ions with laser light and electromagnetic fields has resulted in many of the first key tests of the unique properties of quantum mechanics. For example, Wineland's group was the first in the world to demonstrate a so-called "Schrödinger cat" state. Erwin Schrödinger, a pioneering quantum theorist, illustrated the strangeness of the quantum principle of superposition through the fanciful description in the 1930s of a cat in a closed box that is simultaneously in a state of being dead and alive until an observer opens the box to discover the condition of the cat. In 1996, Wineland conducted the first direct observation of superposition by showing that a single ion simultaneously existed in two states separated by some $80 \mathrm{~nm}$ (much larger than the approximate $0.1 \mathrm{~nm}$ size of the ion) until forced by the measurement process to collapse into one of the states.

\begin{abstract}
A "Schrödinger cat"-like state of matter was generated at the single atom level. A trapped ${ }^{9} \mathrm{Be}^{+}$ ion was laser-cooled to the zero-point energy and then prepared in a superposition of spatially separated coherent harmonic oscillator states. This state was created by application of a sequence of laser pulses, which entangles internal (electronic) and external (motional) states of the ion. The Schrödinger cat superposition was verified by detection of the quantum mechanical interference between the localized wave packets. This mesoscopic system may provide insight into the fuzzy boundary between the classical and quantum worlds by allowing controlled studies of quantum measurement and quantum decoherence.
\end{abstract}




\title{
Solidification sensing using high-energy x-ray diffraction
}

\author{
Thomas A. Siewert, William P. Dubé, and Dale W. Fitting \\ NIST Materials Reliability Division
}

Advanced Materials and Processes, 23-25, July 1996

\section{Significance}

Most x-ray diffraction measurements are performed with conventional low-energy tubes, which limit the depth of penetration to a few micrometers in most materials. As a part of the work under the NIST Aerospace Consortium, we developed high-energy x-ray diffraction procedures (using energies up to $320 \mathrm{kVp}$ ), which penetrate over $20 \mathrm{~mm}$ of aluminum, and so permit x-ray transmission studies. This report summarizes a number of our tests. One of the most interesting was a series of diffraction patterns for a high-purity aluminum rod as it melted and then resolidified. The x-rays were sufficiently energetic to not only penetrate about $10 \mathrm{~mm}$ of aluminum, but also the crucible and furnace walls. Thus, this was the only technique that was found to penetrate the wall and furnace elements of a high-temperature vacuum furnace, and so permit monitoring of the solidification front location and shape during the solidification of single-crystal nickel-alloy turbine blades. The series of patterns in this publication shows a reduction in the number of diffraction spots as the grain structure coarsened during heating, a total loss of spots (and formation of a diffuse diffraction ring) as the aluminum melted, and the reappearance of new spots as the aluminum resolidified. This research served as the basis for U.S. Patent 5,589,690 and was reported in more detail in NIST TN 1500-3.

\begin{abstract}
A high-energy transmission x-ray diffraction technique that combines the capabilities of conventional high- and low-energy x-ray systems has been developed at the National Institute of Standards and Technology as a noncontact sensor for locating the liquid/solid boundary in metal castings. The high-energy (160-320 kV) x-rays can penetrate the thickness of most castings, and the mold and furnace walls. One practical goal for this new method is to collect data on the position, velocity, and possibly the shape of the solidification front of directionally solidified castings. Such information is vital for development and verification of improved solidification models, but it has been difficult to gather because of high temperatures, strong magnetic fields, and vacuum casting furnaces.
\end{abstract}




\title{
X-ray detection using a superconducting transition-edge sensor microcalorimeter with electrothermal feedback
}

\author{
Kent D. Irwin, Gene C. Hilton, David A. Wollman, and John M. Martinis \\ NIST Electromagnetic Technology Division
}

Applied Physics Letters, 69(13): 1945-1947, 1996

\section{Significance}

This paper describes the first practical realization of a new kind of high performance x-ray detector. The transition-edge sensor (TES) microcalorimeter measures x-ray energy thermally, with the absorbed $\mathrm{x}$-ray photon causing a small but measurable temperature increase of the detector. By utilizing a highly sensitive superconducting phase transition as the thermometer, and by operating at low temperatures, this type of detector provides a high resolution measurement of the $\mathrm{x}$-ray energy. The real significance of this paper lies in the new capabilities this type of detector permits. Since this publication, x-ray detectors have been developed using this technique with energy resolution more than 40 times better than conventional instruments. Commercial products based on these devices are now being developed for materials analysis applications, and many groups throughout the world are developing systems based on this technology for nextgeneration x-ray and infrared astronomical instruments.

\begin{abstract}
We have developed a new type of $\mathrm{x}$-ray detector based on a superconducting transition-edge thermometer operated near $100 \mathrm{mK}$. A superconducting quantum interference device is used to measure the current through the thermometer, and negative electrothermal feedback is used to improve the energy resolution and shorten the thermal time constant. We have used a detector mounted on a scanning electron microscope to measure the energy of titanium $\mathrm{K} \alpha(4.5 \mathrm{keV})$ fluorescence $x$ rays with a resolution better than $14 \mathrm{eV}$ full width at half-maximum. Using two other devices, we have measured an energy resolution for Joule heat pulses of $2.6 \mathrm{eV}$ at $1 \mathrm{keV}$ and $0.2 \mathrm{eV}$ at $4 \mathrm{eV}$, the best reported for any calorimeter. An electrical noise equivalent power of $3 \times 10^{-18} \mathrm{~W} / \sqrt{ } \mathrm{Hz}$ was also measured, suggesting the use of these detectors as infrared bolometers.
\end{abstract}




\section{Arctic environmental change of the last four centuries}

\author{
Jonathan T. Overpeck \\ NOAA Paleoclimatology Program, NCDC \\ Konrad Hughen \\ Institute of Arctic and Alpine Research, \\ University of Colorado \\ Douglas Hardy and Raymond Bradley \\ University of Massachusetts \\ Roslyn Case \\ University of California at Los Angeles \\ Maryanne Douglas \\ University of Toronto, Canada \\ Bruce Finney \\ Institute of Marine Sciences \\ University of Alaska \\ Konrad Gajewski \\ University of Ottawa, Canada \\ Gordon Jacoby \\ Lamont-Doherty Earth Observatory \\ Columbia University
}

\author{
Anne Jennings \\ Institute of Arctic and Alpine Research \\ University of Colorado \\ Scott Lamoureux \\ University of Alberta, Canada \\ Andrew Lasca \\ Bates College \\ Glen MacDonald \\ University of California at Los Angeles \\ Jay Moore \\ Institute of Arctic and Alpine Research \\ University of Colorado \\ Michael Retelle \\ Bates College \\ Sheldon Smith \\ University of Massachusetts \\ Alex Wolfe \\ Institute of Arctic and Alpine Research \\ University of Colorado \\ Gregory Zielinski \\ University of New Hampshire
}

Science, 278: 1251-1256, November 14, 1997

\section{Significance}

This paper is among a set of recently published, widely cited papers that document the preinstrumental record of temperature and which have been used to place 20th century warming into a long-term context. A large international team of scientists combined the results of their research to generate the first circum-Arctic view of surface air temperature changes over the last 400 years. The study used proxy climate data from tree-rings, ice-cores, lake and marine sediments. This "multi-proxy" approach insures that results were not biased as is possible if only one proxy source was used. Together, these proxy data allow 20th century warming to be evaluated in the context of the past 400 years. They indicate that the Arctic has warmed up to $1.5^{\circ} \mathrm{C}$ since 1850 - the coolest interval of the Arctic "Little Ice Age." Much of the recent Arctic warming took place between 1850 and 1920, most likely due to natural processes, whereas the warming after 1920 is increasingly difficult to ascribe to natural forcing. The Overpeck et al. results confirm results of earlier regional studies that suggest the Arctic warming is unprecedented in the past four centuries. The authors also discuss the impacts of warming across the Arctic, a region particularly sensitive to warming. These impacts include glacial melting, permafrost warming, lake and forest ecology changes, and the retreating of sea-ice. 


\begin{abstract}
A compilation of paleoclimate records from lake sediments, trees, glaciers, and marine sediments provides a view of circum-Arctic environmental variability over the last 400 years. From 1840 to the mid- $20^{\text {th }}$ century, the Arctic warmed to the highest temperatures in four centuries. This warming ended the Little Ice Age in the Arctic and has caused retreats of glaciers, melting of permafrost and sea ice, and alteration of terrestrial and lake ecosystems. Although warming, particularly after 1920, was likely caused by increases in atmospheric trace gases, the initiation of the arming in the mid- $19^{\text {th }}$ century suggests that increases solar irradiance, decreased volcanic activity, and feedbacks internal to the climate system played roles.
\end{abstract}




\title{
El Niño, La Niña, and the nonlinearity of their teleconnections
}

\author{
Martin P. Hoerling \\ NOAA/CIRES Climate Diagnostics Center \\ Arun Kumar \\ NOAA National Center for Environmental Prediction \\ Min Zhong \\ NOAA/CIRES Climate Diagnostics Center
}

Journal of Climate, 10(10): 1769-1786, 1997

\section{Significance}

This heavily cited paper showed that the paradigm of "equal and opposite" atmospheric responses to El Niño and La Niña conditions was fundamentally incorrect. It provided an explanation for the different characteristics of the responses to El Niño and La Niña forcings over the North American region. As such, this paper paved the way for significant improvements in NOAA forecasts of the climate response to El Niño and La Niña.

\begin{abstract}
The paradigm of an atmospheric system varying linearly with respect to extreme phases of the El Niño-Southern Oscillation is questioned. It is argued that the global response to tropical Pacific sea surface temperature forcing will be inherently nonlinear. A physical basis for this intrinsic nonlinearity is the thermodynamic control on deep convection.

Climate statistics for warm and cold events of the tropical Pacific are analyzed separately for the northern winter periods during 1950-96. Composite analysis of 500-mb heights reveal planetaryscale teleconnection patterns, as noted in earlier studies. A new result is the evidence for an appreciable $35^{\circ}$ longitude phase shift between the warm and cold event circulation composites, and the two wave trains appear to have different tropical origins. A large nonlinear component in North American surface climate anomalies is also found, which is consistent with such a phase shift in teleconnections. In the Tropics, rainfall anomalies also show evidence of nonlinear behavior. The maximum rain anomalies along the equator are located east of the date line during warm events, but west of the date line during cold events. The interpretation of this behavior is complicated, however, by the fact that composite warm event SST anomalies are not the exact inverse of their cold event counterparts.

Idealized atmospheric general circulation model (AGCM) experiments are performed in order to test the question of whether the observed nonlinearity is an intrinsic property of the atmospheric system. The model is forced with a composite SST anomaly that undergoes a realistic seasonally varying ENSO life cycle, as described by E. Rasmusson and T. Carpenter. Both positive and negative phases of the SST anomaly are used, and a 40-member ensemble of warm and cold event model simulations is conducted. A nonlinear climate response in the AGCM is found that closely resembles the observed composites, including a shift in the equatorial positions of the maximum rain responses and a phase shift of teleconnection patterns in the upper troposphere.
\end{abstract}


Barotropic model experiments indicate that the inherent nonlinearity in the tropical rain response may itself be responsible for the phase shift in the extratropical teleconnection patterns. 


\title{
Isochoric $(p, \rho, T)$ measurements for five natural gas mixtures from $\mathrm{T}=(225$ to 350$) \mathrm{K}$ at pressures to $35 \mathrm{MPa}$
}

Joseph W. Magee, William M. Haynes, and Michael J. Hiza

NIST Physical and Chemical Properties Division

Journal of Chemical Thermodynamics, 29: 1439-1454, 1997

\section{Significance}

Experimental measurements on natural gas properties are necessary to establish the wide ranging and flexible models used to describe these high-energy-content fluids. The program at NIST was geared to establishing a validated set of measurements on key mixtures with density determined at an expanded uncertainty of $0.05 \%$. The techniques used, mainly an isochoric gas expansion method on gravimetrically prepared mixtures, had been well established at NIST. The measurements described in this paper form the basis for the models used in our 100 billion dollar natural gas industry.

\begin{abstract}
Measurements of the $(\mathrm{p}, \rho, \mathrm{T})$ properties of five natural gas mixtures were carried out at temperatures from (225 to 350 ) $\mathrm{K}$ at pressures to $35 \mathrm{MPa}$ using an isochoric gas expansion apparatus. Ten isochores have been determined for each mixture at densities ranging from $1 \mathrm{~mol}$ $\cdot \mathrm{dm}^{-3}$ to $16 \mathrm{~mol} \cdot \mathrm{dm}^{-3}$. The gas samples were prepared gravimetrically from pure gases of verified research-grade purity. The uncertainty of the density measurements arises primarily from uncertainties involved in the determination of the experimental cell volume and of the amount of substance contained in the experimental volume. When all components of experimental uncertainty arising from random effects are considered, the expanded uncertainty (comprising 95 per cent confidence interval) of the density measurements is estimated to be 0.044 per cent.
\end{abstract}




\title{
Mapping city lights with nighttime data from the DMSP Operational Linescan System
}

\author{
Christopher D. Elvidge \\ NOAA National Geophysical Data Center \\ Kimberly E. Baugh \\ NOAA/CIRES \\ Eric A. Kihn and Herbert W. Kroehl \\ NOAA National Geophysical Data Center \\ Ethan R. Davis \\ NOAA/CIRES
}

Photogrammetric Engineering and Remote Sensing, 63(6), 727-734, 1997

\section{Significance}

This paper received the outstanding paper of the year award issued by the Royal Society. It defines the method used to detect stable and ephemeral sources of "nighttime lights" from imagery designed to detect clouds. The methodology has been used to estimate the population of countries, the economic vitality of regions, increases in urban sprawl, and local amounts of carbon emissions.

\begin{abstract}
The Defense Meteorological Satellite Program (DMSP) Operational Linescan System (OLS) has a unique capability to detect low levels of visible and near-infrared (NVIR) radiance at night. With the OLS "VIS" band data, it is possible to detect clouds illuminated by moonlight, plus lights from cities, towns, industrial sites, gas flares, and ephemeral events such as fires and lightning illuminated clouds. This paper presents methods which have been developed for detecting and geolocating VNIR emission sources with nighttime DMSP-OLS data and the analysis of image time series to identify spatially stable emissions from cities, towns and industrial sites. Results are presented for the United States.
\end{abstract}




\title{
Electromagnetic theory of reverberation chambers
}

\author{
David A. Hill \\ NIST Radio-Frequency Technology Division
}

NIST Technical Note 1506, 1998

\section{Significance}

Reverberation chambers have become very popular worldwide for EMC immunity and emissions measurements. This Tech Note provides the necessary theory for performing such measurements. It is the only publication that simultaneously provides both electromagnetic and statistical theory for predicting field properties and test object responses. Hence, it has been heavily used and referenced worldwide. The U.S. Air Force has adopted it as its standard reference document for reverberation chamber measurements in house and by contractors.

\begin{abstract}
This report presents the electromagnetic theory of reverberation chambers as applied to electromagnetic compatibility measurements. For radiated immunity measurements, mode theory and the plane-wave integral representation are useful in determining electric and magnetic field characteristics and chamber quality factor $(Q)$. The plane-wave integral representation also provides a convenient method for describing the response of a reference antenna or a test object in a stirred field. For radiated emissions, energy conservation or electromagnetic reciprocity can be used to describe the measurement of total radiated power. Comparisons of theory and measurements are presented for chamber $Q$, probability density functions of fields and received power, and radiated immunity and emissions of a simple test object. Recommendations are made for improvements in statistical electromagnetic theory as applied to mechanical stirring and the statistics of immunity and emissions measurements.
\end{abstract}




\title{
2000 years of drought variability in the central United States
}

\author{
Connie A. Woodhouse and Jonathan T. Overpeck \\ NOAA National Climatic Data Center, Paleoclimatology Program
}

Bulletin of the American Meteorological Society, 79: 2693-2714, 1998

\section{Significance}

Our understanding of drought is based on the 20th century records of climate, which contain only a subset of the range of climatic variability that can be expected under naturally occurring climate conditions. This paper assessed the results of several studies documenting drought in the central and western United States over the past 2000 years, based on a wide range of paleoclimatic data. These studies indicated that the severity of droughts of the 20th century was eclipsed several times by droughts in the previous 2000 years. Of particular note is a remarkably widespread and persistent "megadrought" at the end of the 16th century. If the climate of the past can be considered a guide to what may be expected in the future, this work suggests that droughts more severe than those of the 1930s and 1950s are likely to occur in the future, a likelihood that might be exacerbated by greenhouse warming. These results have important implications for water resource management, particularly in the arid and semi-arid regions of the United States.

\begin{abstract}
Droughts are one of the most devastating natural hazards faced by the United States today. Severe droughts of the twentieth century have had large impacts on economies, society, and the environment, especially in the Great Plains. However, the instrumental record of the last 100 years contains only a limited subset of drought realizations. One must turn to the paleoclimatic record to examine the full range of past drought variability, including the range of magnitude and duration, and thus gain the improved understanding needed for society to anticipate and plan for droughts of the future. Historical documents, tree rings, archaeological remains, lake sediment, and geomorphic data make it clear that the droughts of the twentieth century, including those of the 1930s and 1950s, were eclipsed several times by droughts earlier in the last 2000 years, and as recently as the late sixteenth century. In general, some droughts prior to 1600 appear to be characterized by longer duration (i.e., multidecadal) and greater spatial extent than those of the twentieth century. The authors' assessment of the full range of past natural drought variability, deduced from a comprehensive review of the paleoclimatic literature, suggests that droughts more severe than those of the 1930s and 1950s are likely to occur in the future, a likelihood that might be exacerbated by greenhouse warming in the next century. Persistence conditions that lead to decadal-scale drought may be related to low-frequency variations, or base-state shifts, in both the Pacific and Atlantic Oceans, although more research is needed to understand the mechanisms of severe drought.
\end{abstract}




\title{
A capacitance standard based on counting electrons
}

\author{
Mark W. Keller, Ali L. Eichenberger, John M. Martinis, and Neil M. Zimmerman \\ NIST Electromagnetic Technology Division
}

Science, 285: 1706-1709, 1999

\section{Significance}

Once precise electron counting was demonstrated in 1994, the question became whether or not it could be put to practical use. Five years later, Mark Keller, Ali Eichenberger, John Martinis, and Neil Zimmerman proved the case by building a fully practical standard for the measurement of electrical capacitance. Their measurement technique required pumping an exact number of electrons onto a capacitor, around 50 million, then accurately measuring the capacitor's voltage without disturbing more than a few electrons. Their new technique is accurate to better than 0.3 parts per million and will provide an important check on established methods of capacitance calibration. It will also yield an independent measure of the fine structure constant, one of nature's fundamental numbers.

\begin{abstract}
A capacitance standard based directly on the definition of capacitance was built. Single-electron tunneling devices were used to place $N$ electrons of charge $e$ onto a cryogenic capacitor $C$, and the resulting voltage change $\Delta V$ was measured. Repeated measurements of $C=N e / \Delta V$ with this method have a relative standard deviation of $0.3 \times 10^{-6}$. This standard offers a natural basis for capacitance analogous to the Josephson effect for voltage and the quantum Hall effect for resistance.
\end{abstract}




\title{
For 100 years, notched bar impact testing standards have yielded widespread benefits for industry
}

Michael P. Manahan, Chris N. McCowan, Thomas A. Siewert, John M. Holt, Francis J. Marsh, and Earl A. Ruth

NIST Materials Reliability Division

Standardization News, 27(2): 30-35, February 1999

\section{Significance}

This paper was written to recognize the centenary of the pendulum impact test, now most commonly used in a configuration known as the Charpy impact test. The paper described the early history of the test, the development of standard methods that reduce the uncertainty in the measurements, and the importance of the technique in the steel fabrication industries (including many fracture critical structures such as bridges, ships, and buildings). This paper received an award from the American Society for Testing and Materials during their centennial celebration in 1998 , in recognition of the importance of impact standards in promoting public safety and reducing the costs associated with material variation.

\begin{abstract}
Without standardization, the impact test has little meaning. The test result obtained from an impact test is dependent upon the specimen size, notch geometry, and the geometry of the anvils and striker. To a lesser degree, impact test results are also dependent upon other variables such as impact velocity, energy lost to the test machine, and friction. The goal of those who have written and modified ASTM E23, Standard Test Methods for Notched Bar Impact Testing of Metallic Materials, has been, and is to standardize and control the variables associated with impact testing.
\end{abstract}




\title{
Objective estimation of perceived speech quality Part I: Development of the measuring normalizing block technique Part II: Evaluation of the measuring normalizing block technique
}

\author{
Stephen Voran \\ NTIA Institute for Telecommunication Sciences
}

IEEE Transactions on Speech and Audio Processing, 7(4): 371-382 and 383-390, July 1999

\section{Significance}

This set of two papers presents a substantial innovation and practical advancement in the objective estimation of transmitted speech quality. The first paper defines the mathematical approach, which comprises a simple but effective perceptual transformation and a hierarchy of measuring normalizing blocks, each of which integrates two perceptually transformed signals over a defined time or frequency interval to obtain an objective difference measure that can be correlated with subjective (human listener) speech quality evaluations. The second paper thoroughly evaluates the resulting new estimators as well as other established metrics. The defined estimators have been standardized, patented, licensed, and implemented in commercial test equipment.
Abstract, Part I
Perceived speech quality is most directly measured by subjective listening tests. These tests are often slow and expensive, and numerous attempts have been made to supplement them with objective estimators of perceived speech quality. These attempts have found limited success, primarily in analog and higher-rate, error-free digital environments where speech waveforms are preserved or nearly preserved. The objective estimation of the perceived quality of highly compressed digital speech, possibly with bit errors or frame erasures has remained an open question. We report our findings regarding two essential components of objective estimators of perceived speech quality: perceptual transformations and distance measures. A perceptual transformation modifies a representation of an audio signal in a way that is approximately equivalent to the human hearing process. A distance measure reflects the magnitude of a perceived distance between two perceptually transformed signals.

We then describe a new objective estimation approach that uses a simple but effective perceptual transformation and a distance measure that consists of a hierarchy of measuring normalizing blocks. Each measuring normalizing block integrates two perceptually transformed signals over some time or frequency interval to determine the average difference across that interval. This difference is then normalized out of one signal, and is further processed to generate one or more measurements. The resulting new estimators, and several established estimators, are thoroughly evaluated and compared in Part II of this paper. Hierarchical structures of measuring normalizing blocks, or other structures of measuring normalizing blocks may also address open issues in perceived audio quality estimation, layered speech or audio coding, automatic speech or speaker recognition, audio signal enhancement, and other areas. 


\begin{abstract}
Part II
Part I of this paper describes a new approach to the objective estimation of perceived speech quality. This new approach uses a simple but effective perceptual transformation and a distance measure that consists of a hierarchy of measuring normalizing blocks. Each measuring normalizing block integrates two perceptually transformed signals over some time or frequency interval to determine the average difference across that interval. This difference is then normalized out of one signal, and is further processed to generate one or more measurements. In Part II, the resulting estimates of perceived speech quality are correlated with the results of nine subjective listening tests. Together, these tests include $2194-\mathrm{kHz}$ bandwidth speech codecs, transmission systems, and reference conditions, with bit rates ranging from 2.4 to $64 \mathrm{~kb} / \mathrm{s}$. When compared with six other estimators, significant improvements are seen in many cases, particularly at lower bit rates, and when bit errors or frame erasures are present. These hierarchical structures of measuring normalizing blocks, or other structures of measuring normalizing blocks may also address open issues in perceived audio quality estimation, layered speech or audio coding, automatic speech or speaker recognition, audio signal enhancement, and other areas.
\end{abstract}




\section{Papers from the 2000s}

Pressure-induced shift and broadening of 1510-1540 acetylene wavelength calibration lines William C. Swann and Sarah L. Gilbert, 2000

An optical clock based on a single trapped $\mathrm{Hg}+$ ion Scott A. Diddams, Thomas Udem, Kurt R. Vogel, Chris W. Oates, E.A. Curtis, W. David Lee, Wayne M. Itano, Robert E. Drullinger, James C. Bergquist, and Leo Hollberg, 2001

Weather radar for remote sensing:

1. On the use of radar depolarization ratios for estimating shapes of ice hydrometeors in winter clouds

Sergey Matrosov, Roger Reinking, Robert Kropfli, Brooks Martner, and Bruce Bartram, 2001

2. Evaluation of a 45-deg slant quasi-linear radar polarization state for distinguishing drizzle droplets, pristine ice crystals, and less regular ice particles

Roger Reinking, Sergey Matrosov, Robert Kropfli, and Bruce Bartram, 2002

The temporal and spectral characteristics of ultrawideband signals

William A. Kissick, 2001

Tropical origins for North Atlantic climate change

Martin P. Hoerling, Taiyi Xu, and James W. Hurrell, 2001

Wideband model of HF atmospheric radio noise

John J. Lemmon, 2001

Accuracy evaluation of NIST-F1

Steven R. Jefferts, Jon H. Shirley, Thomas E. Parker, Thomas P. Heavner, Dawn M.

Meekhof, Craig W. Nelson, Robert E. Drullinger, Leo Hollberg, W. David Lee,

Fred L. Walls, Filippo Levi, Giovanni Costanzo, and Andrea DeMarchi, 2002

Increase in the Asian SW monsoon during the past four centuries

David M. Anderson, Jonathan T. Overpeck, and Anil K. Gupta, 2002

On determining the maximum emissions from electrically large sources

Perry F. Wilson, David A. Hill, and Christopher L. Holloway, 2002

Video quality measurement techniques

Stephen Wolf and Margaret Pinson, 2002

The perfect ocean for drought

Martin P. Hoerling and Arun Kumar, 2003 


\title{
Pressure-induced shift and broadening of 1510-1540 acetylene wavelength calibration lines
}

\author{
William C. Swann and Sarah L. Gilbert \\ NIST Optoelectronics Division
}

Journal of the Optical Society of America B, 17: 1263-1270, 2000

\section{Significance}

This paper provided the technical basis for the first of a family of Standard Reference Material (SRM) wavelength standards for optical communications. During the development of wavelength-division-multiplexed (WDM) optical communications systems, these SRMs were in great demand; nearly 200 have been sold, and they are used as primary standards in most laboratories involved in optical communications throughout the world. Similar standards have been incorporated into commercial optical spectrum analyzers.

\begin{abstract}
We have measured the line centers and the pressure-induced shifts of 14 lines in the $3 v$ rotational-vibrational band of carbon monoxide, $12 \mathrm{C} 160$, and 18 lines in the corresponding band of ${ }^{13} \mathrm{C}^{16} \mathrm{O}$. These lines can be used as wavelength references in the optical fiber communication wavelength-division multiplexing $L$ band (approximately $1565-1625 \mathrm{~nm}$ ). The ${ }^{12} \mathrm{C}^{16} \mathrm{O}$ spectrum has useful reference lines from 1560 to $1595 \mathrm{~nm}$, and the ${ }^{13} \mathrm{C}^{16} \mathrm{O}$ spectrum has lines from 1595 to $1630 \mathrm{~nm}$. We observed that, except for a shift of $35 \mathrm{~nm}$ toward longer wavelengths for the ${ }^{13} \mathrm{C}^{16} \mathrm{O}$ spectrum, the behaviors of the two isotopic species are similar. We found that the pressure shift varies with line number, from $\sim+0.01$ to $+0.02 \mathrm{pm} / \mathrm{kPa}(\sim 0.16$ to $0.31 \mathrm{MHz} / \mathrm{Torr})$. In addition, we measured the pressure broadening of these lines; we found that it also varies with line number and is typically $0.3-0.4 \mathrm{pm} / \mathrm{kPa}$ (approximately $5-7 \mathrm{MHz} / \mathrm{Torr}$ ).
\end{abstract}




\title{
An optical clock based on a single trapped $\mathrm{Hg}+$ ion
}

\author{
Scott A. Diddams, Thomas Udem, Kurt R. Vogel, Chris W. Oates, E.A. Curtis, W. David Lee, \\ Wayne M. Itano, Robert E. Drullinger, James C. Bergquist, and Leo Hollberg \\ NIST Time and Frequency Division
}

Science, 293: 825-293, August 3, 2001

\section{Significance}

While the uncertainty of the current NIST-F1 laser-cooled atomic fountain clock is equivalent to about 1 second in 30 million years, there is a fundamental limitation on improving its accuracy: it uses a microwave transition dividing the second into approximately 9.2 billion "ticks." Using a transition with many more "ticks" per second enables the second to be more accurately realized. This paper describes an atomic clock based on a transition in a laser-cooled and trapped mercury ion at $282 \mathrm{~nm}$ (ultraviolet), dividing the second into about 1.1 million billion "ticks," with the potential to be up to 1,000 times more accurate than the NIST-F1 clock. This paper also demonstrates both a strong candidate for a future optical clock to replace the cesium-atom-based standard, and the means for accurately disseminating time and frequency standards from it. The microwave standard is relatively easy to disseminate, but the optical frequency standard is not. The paper describes the use of an optical frequency "comb" to directly link optical transitions to microwave transitions. The "comb" consists of an array of very sharp laser lines spanning a broad spectral range, and differing in frequency from each other by a precise and controllable amount, like the teeth of a comb. The researchers demonstrated that the mercury ion transition at about $1.1 \times 1015 \mathrm{~Hz}$ could be directly compared to a sub-multiple of the cesium atom atomic clock transition. Improvement in atomic clocks has led to transforming commercial applications, such as the Global Positioning System and cellular communications networks, and to new scientific applications such as precision tests of quantum mechanics, relativity, and astrophysics.

\begin{abstract}
Microwave atomic clocks have been the de facto standards for precision time and frequency metrology over the past 50 years, finding widespread use in basic scientific studies, communications, and navigation. However, with its higher operating frequency, an atomic clock based on an optical transition can be much more stable. We demonstrate an all-optical atomic clock referenced to the 1.064-petahertz transition of a single trapped ${ }^{199} \mathrm{Hg}^{+}$ion. A clockwork based on a mode-locked femtosecond laser provides output pulses at a 1-gigahertz rate that are phase-coherently locked to the optical frequency. By comparison to a laser-cooled calcium optical standard, an upper limit for the fractional frequency instability of $7 \times 10^{-15}$ is measured in 1 second of averaging - a value substantially better than that of the world's best microwave atomic clocks.
\end{abstract}




\title{
Weather radar for remote sensing:
}

\section{On the use of radar depolarization ratios for estimating shapes of ice hydrometeors in winter clouds}

\author{
Sergey Matrosov \\ NOAA Environmental Technology Laboratory/CIRES \\ Roger Reinking, Robert Kropfli, Brooks Martner, and Bruce Bartram \\ NOAA Environmental Technology Laboratory
}

Journal of Applied Meteorology, 40: 479-490, 2001

\section{Evaluation of a 45-deg slant quasi-linear radar polarization state for distinguishing drizzle droplets, pristine ice crystals, and less regular ice particles}

Roger Reinking, Sergey Matrosov, Robert Kropfli, and Bruce Bartram

NOAA Environmental Technology Laboratory

Journal of Atmospheric and Oceanic Technology, 19: 296-321, 2002

\section{Significance}

These companion papers, which unite new theory (Matrosov et al.) and corresponding experimental verification (Reinking et al.), represent the culmination of a 10-year series of specialized weather radar development efforts to devise methods and hardware that identify cloud hydrometeors by their physical characteristics in order to isolate by remote sensing those types of particles that cause aircraft icing. These efforts and publications resulted in a 2001 U.S. Department of Commerce Gold Medal Award, and the Federal Aviation Administration's Administrator's 2002 Excellence in Aviation Award.

\begin{abstract}
1
An approach is suggested to relate measurements of radar depolarization ratios and aspect ratios of predominant hydrometeors in nonprecipitating and weakly precipitating layers of winter clouds. The trends of elevation angle dependencies of depolarization ratios are first used to distinguish between columnar-type and plate-type particles. For the established particle type, values of depolarization ratios observed at certain elevation angles, for which the influence of particle orientation is minimal, are then used to estimate aspect ratios when the information on particle effective bulk density is assumed or inferred from other measurements. The use of different polarizations, including circular, slant $-45^{\circ}$ linear, and two elliptical polarizations is discussed. These two elliptical polarizations are quasi-circular and quasi-slant $-45^{\circ}$ linear, and both are currently achievable with the National Oceanic and Atmospheric Administration Environmental Technology Laboratory's $\mathrm{K}_{\mathrm{a}}$-band radar. In comparison with the true circular and true slant $-45^{\circ}$ linear polarizations, the discussed elliptical polarizations provide a stronger signal
\end{abstract}


in the "weak" radar receiving channel; however, it is at the expense of diminished dynamic range of depolarization ratio variations. For depolarization measurements at the radar elevation angles that do not show much sensitivity to particle orientations, the available quasi-circular polarization provides a better depolarization contrast compared to the available quasi-slant-4So linear polarization. The use of the proposed approach is illustrated with the experimental data collected during a recent field experiment. It is shown that it allows successful differentiation among pristine planar crystals, rimed planar crystals, long columns, blocky columns, and graupel. When a reasonable assumption about particle bulk density is made, quantitative estimates of particle aspect ratios from radar depolarization data are in good agreement with in situ observations. This approach can be used to detect super-cooled liquid droplets.

\section{Abstract 2}

A remote sensing capability is needed to detect clouds of super-cooled, drizzle-sized droplets, which are a major aircraft icing hazard. Discrimination among clouds of differing ice particle types is also important because both the presence and type of ice influence the survival of liquid in a cloud and the chances for occurrence of these large, most hazardous droplets. This work shows how millimeter-wavelength dual-polarization radar can be used to identify these differing hydrometeors. It also shows that by measuring the depolarization ratio (DR), the estimation of the hydrometeor type can be accomplished deterministically for drizzle droplets; ice particles of regular shapes; and to a considerable extent, the more irregular ice particles, and that discrimination is strongly influenced by the polarization state of the transmitted microwave radiation. Thus, appropriate selection of the polarization state is emphasized.

The selection of an optimal polarization state involves trade-offs in competing factors such as the functional dynamic range of DR, sensitivity to low-reflectivity clouds, and insensitivity to oscillations in the settling orientations of ice crystals. A $45^{\circ}$ slant, quasi-linear polarization state, one in which only slight ellipticity is introduced, was found to offer a very good compromise, providing considerable advantages over standard horizontal and substantially elliptical polarizations. This was determined by theoretical scattering calculations that were verified experimentally in field measurements conducted during the Mount Washington Icing Sensors Project (MWISP). A selectable-dual-polarization Ka-band (8.66 mm wavelength) radar was used. A wide variety of hydrometeor types was sampled. Clear differentiation among planar crystals, columnar crystals, and drizzle droplets was achieved. Also, differentiation among crystals of fundamentally different shapes (aspect ratios) within each of the planar and columnar families was found possible. These distinctions matched calculations of DR, usually to within 1 or $2 \mathrm{~dB}$. The results from MWISP and from previous experiments with other polarizations have demonstrated that the agreement between theory and measurements by this method is repeatable. Additionally, although less rigorously predicted by theory, the field measurements demonstrated substantial differentiation among the more irregular and more spherical ice particles, including aggregates, elongated aggregates, heavily rimed dendrites, and graupel. Measurable separation between these various irregular ice particle types and drizzle droplets was also verified. 


\title{
The temporal and spectral characteristics of ultrawideband signals
}

\author{
William A. Kissick \\ NTIA Institute for Telecommunication Sciences \\ NTIA Report 01-383, January 2001
}

\section{Significance}

This report broke new ground in the understanding of how ultrawideband (UWB) signals can be measured, characterized, and regulated, and how they interfere with traditional narrowband radio services. With nanosecond pulse widths and bandwidths that can span several $\mathrm{GHz}$ of spectrum, UWB devices (for radio communications, sensing, and imaging) spread their energy "thinly" across many bands of the radio spectrum. Because these bands are in use by other services, UWB represents a great challenge to spectrum management. To study UWB devices, new measurement techniques had to be used, and new analysis techniques developed. Performed at a time of widespread erroneous technical claims about UWB interference, this report provided the first comprehensive technical basis for subsequent UWB work by many other researchers. It is expected to become the "classic" for measurement and characterization of UWB signals. No report of similar thoroughness has been produced by other researchers, nor is one expected.

\begin{abstract}
Ultrawideband (UWB) technology, useful for both communication and sensing applications, uses the radio spectrum differently than the vast majority of radiocommunication technologies. UWB systems make use of narrow pulses and time-domain signal processing. Questions regarding how these systems, with their potentially very wide emission bandwidths, might affect the efficient use of the radio spectrum or cause interference to conventional radio and wireless systems must be answered before there is any large-scale deployment of UWB systems. The investigation reported here examined both the temporal and spectral characteristics of UWB signals, since all radio signals exist in both the time and frequency domains. The investigation was approached with theoretical analyses, measurement of actual UWB devices, and computer simulations. The emissions of several UWB transmitters were measured under controlled, and repeatable, laboratory conditions. Those measurement methods useful for routine measurements using commercially available test equipment were identified. The characteristics of an aggregate of several UWB signals were examined. An initial assessment of the effects of UWB signals on several Federal Government systems was accomplished through field measurements. This report provides a basis for an assessment of the effects of UWB signals on other communication and radar systems, the study of the spectrum efficiency of UWB technologies, and the development of spectrum sharing policies and regulations.
\end{abstract}




\title{
Tropical origins for North Atlantic climate change
}

\author{
Martin P. Hoerling and Taiyi Xu \\ NOAA/CIRES Climate Diagnostics Center \\ James W. Hurrell \\ National Center for Atmospheric Research
}

Science, 292: 90-92, 2001

\section{Significance}

The paper shows that North Atlantic climate change over recent decades is consistent with a warming trend of the tropical Indian and Pacific oceans. It thus opened an entirely new focus of future research inquiry on the nature of the link between the tropical eastern hemisphere oceans - the warm pool in particular - and North Atlantic climate. The theory put forth is that this change is a regional fingerprint of anthropogenic influence, and it thereby provides a scientific rationale for projecting a sustained, future climatic disruption over the North Atlantic/Eurasian sector. The paper offers compelling basic scientific evidence for the origin of recent climate change over the North Atlantic, identifying the agent and the process forcing decadal climate change. It improves climate information on the joint environmental change of oceans and climate, thus providing a new scientific basis for decision and policy-making. It also provides a new basis for improved climate predictions of North Atlantic climate and its environs, based on the impact of the Indo-Pacific Ocean. In so doing, it offers a new tool for both seasonal and decadal climate prediction, augmenting the well-known El Niño predictability. This widely cited publication has helped motivate new scientific efforts, including an enhanced observing system over the Indo-Pacific Ocean, to capitalize on the potential climate predictability.

\footnotetext{
Abstract

Evidence is presented that North Atlantic climate change since 1950 is linked to a progressive warming of tropical sea surface temperatures, especially over the Indian and Pacific Oceans. The ocean changes alter the pattern and magnitude of tropical rainfall and atmospheric heating, the atmospheric response to which includes the spatial structure of the North Atlantic Oscillation (NAO). The slow, tropical ocean warming has thus forced a commensurate trend toward one extreme phase of the NAO during the past half-century.
} 


\title{
Wideband model of HF atmospheric radio noise
}

John J. Lemmon

NTIA Institute for Telecommunication Sciences

Radio Science, 36(6): 1385-1391, November-December 2001

\section{Significance}

This paper presents an original model for simulating high-frequency (HF) atmospheric radio noise, essential for predicting the behavior of communications systems in the HF bands. It is part of a research program at ITS dating back to the 1970s that included the development of a channel simulator to test HF radio performance in the laboratory, and models of actual propagation as well as this model of atmospheric noise and interference, which can be a real problem in the congested HF bands. Communications technologies such as spread spectrum and advanced digital signal processing techniques are being applied to HF wideband communications systems, which, because they are covert (almost undetectable), have military applications. Since many uncertainties exist concerning the performance of these systems, accurate noise models are essential. The methodology presented in this paper provides a strong foundation for testing and evaluation for new systems well into the future.

\begin{abstract}
A model of the waveform generated by high-frequency atmospheric radio noise is presented. Cumulative probability distributions of the noise envelope are derived and shown to be in good agreement with a large database collected from a wide range of noise environments. The model includes correlations in the waveforms that simulate the burst structure of measured atmospheric noise. The bandwidth dependence of the voltage deviation parameter, which parameterizes the impulsiveness of the noise, shows behavior that is qualitatively similar to a limited amount of measured data.
\end{abstract}




\title{
Accuracy evaluation of NIST-F1
}

Steven R. Jefferts, Jon H. Shirley, Thomas E. Parker, Thomas P. Heavner, Dawn M. Meekhof, Craig W. Nelson, Robert E. Drullinger, Leo Hollberg, W. David Lee, and Fred L. Walls NIST Time and Frequency Division

Filippo Levi Instituto Elettrotecnico Nazionale, Italy

Giovanni Costanzo and Andrea DeMarchi

Politecnico di Torino, Italy

Metrologia, 39: 321-336, January 1, 2002

\section{Significance}

This paper describes the current U.S. national standard for time and frequency, the NIST-F1 laser-cooled atomic fountain clock. NBS created the world's first atomic clock in 1949, based on transitions in ammonia molecules. In 1952, NBS adapted the science and technology to beams of cesium atoms, and through the 1990s continually improved the performance of atomic clocks based on cesium beams. But using thermal beams with relatively hot atoms causes a fundamental limit to how precisely the measurements can be made because the rapidly moving atoms have significant Doppler shifts and can only be observed for a relatively short time. After NBS's first demonstration of laser-cooling in 1978, it was realized that atomic clock accuracy could be greatly improved by cooling the atoms to near absolute zero, drastically reducing the Doppler shift and enabling much longer measurement times. This paper describes the first full characterization and uncertainty analysis for NIST's first laser-cooled atomic fountain clock, NIST-F1. When first operated in 1999, NIST-F1 had an uncertainty equivalent to about 1 second in 15 million years, and it has improved to about 1 second in 30 million years. The improvement of atomic clocks over the past decades, with accuracy improving by about a factor of 10 per decade, has enabled many transforming commercial and scientific applications.

\begin{abstract}
The evaluation procedure of a new laser-cooled caesium fountain primary frequency standard developed at the National Institute of Standards and Technology (NIST) is described. The new standard, NIST-F1, is described in some detail and typical operational parameters are discussed. Systematic frequency biases for which corrections are made-second-order Zeeman shift, blackbody radiation shift, gravitational red shift and spin-exchange shift — are discussed in detail. Numerous other frequency shifts are evaluated, but are so small in this type of standard that corrections are not made for their effects. We also discuss comparisons of this standard both with local frequency standards and with standards at other national laboratories.
\end{abstract}




\title{
Increase in the Asian SW monsoon during the past four centuries
}

\author{
David M. Anderson and Jonathan T. Overpeck \\ NOAA National Climatic Data Center, Paleoclimatology Program \\ Anil K. Gupta \\ Indian Institute of Technology, Khragpur, India
}

Science, 297: 596-599, 2002

\section{Significance}

This record is the first paleo reconstruction of the monsoon during the last millennium, is five times longer than the 140-year instrumental record of the monsoon, and reveals centennial scale variability in addition to a long term trend. The research is significant because it supports the idea that the monsoon winds may increase in coming decades as the northern hemisphere continues to warm. These changes may affect a vast region of heavily populated Asia, with significant impacts on agriculture and other industries. This report and a companion paper received significant media attention, including National Public Radio and British Broadcasting Service interviews, a National Geographic television interview, and a perspectives review in Science. The papers had 6 citations each in the Science Citation Index within the first 12 months.

\begin{abstract}
Climate reconstructions reveal unprecedented warming in the past century; however, little is known about trends in aspects such as the monsoon. We reconstructed the monsoon winds for the past 1000 years using fossil Globigerina bulloides abundance in box cores from the Arabian Sea and found that monsoon wind strength increased during the past four centuries as the Northern Hemisphere warmed. We infer that the observed link between Eurasian snow cover and the southwest monsoon persists on a centennial scale. Alternatively, the forcing implicated in the warming trend (volcanic aerosols, solar output, and greenhouse gases) may directly affect the monsoon. Either interpretation is consistent with the hypothesis that the southwest monsoon strength will increase during the coming century as greenhouse gas concentrations continue to rise and northern latitudes continue to warm.
\end{abstract}




\title{
On determining the maximum emissions from electrically large sources
}

\author{
Perry F. Wilson, David A. Hill, and Christopher L. Holloway \\ NIST Radio-Frequency Technology Division
}

IEEE Transactions on Electromagnetic Compatibility, EMC-44(1): 79-86, February 2002

\section{Significance}

This paper develops theory based estimates for the directivity of an unintentional emitter. The directivity estimate can be combined with straightforward measurements of the total radiated power from a device to estimate the maximum emissions from the device and hence potential of the device to cause electromagnetic interference. Numerical simulations and measured data show the effectiveness of the approach. The results presented in this paper directly impact electromagnetic compatibility (EMC) testing of devices whose dimensions are large compared to a wavelength. The results also directly impact the development of EMC standards for frequencies above $1 \mathrm{GHz}$ (the present upper bound for most EMC emissions testing). The results in the paper should lead to reduced test times. Reduced test times will have a significant economic impact because of existing requirements that all digital devices sold in the US, the European Community, and many other world markets must satisfy emission test limits. The paper was awarded the 2003 IEEE EMC Transactions Prize Paper Award (for papers published in 2002) and the 2002 EEEL Outstanding Authorship Award.

\begin{abstract}
This paper examines the use of measurements of the total radiated power from an arbitrary source, combined with theory-based estimates of directivity or quality factor, to accurately predict the maximum electric field radiated by the source either at a line-of-sight distance or in a resonant volume. The approach using total radiated power avoids source rotations during testing and is an efficient alternative to current emission test methods. Examples of simulated (random source set) and measured (box with holes) planar-cut radiation-patterns for electrically large sources are presented. Both simulated and measured data agree well with theoretical estimates.
\end{abstract}




\title{
Video quality measurement techniques
}

\author{
Stephen Wolf and Margaret Pinson \\ NTIA Institute for Telecommunication Sciences
}

NTIA Report 02-392, June 2002

\section{Significance}

This NTIA Report describes an ITS-developed objective video quality metric (VQM) capable of accurately predicting the subjective (human viewer) evaluations of transmitted video signals for a wide range of source video characteristics (e.g., spatial detail, motion) and system impairments

(e.g., compression, transmission error, data loss). The VQM has been standardized nationally and internationally, patented, licensed, and implemented in computer-based test equipment. The underlying mathematical algorithms are highly innovative, and the computer-based techniques for emulating human perception and judgment first described here are now accepted worldwide.

\begin{abstract}
Objective metrics for measuring digital video performance are required by Government and industry for specification of system performance requirements, comparison of competing service offerings, service level agreements, network maintenance, and optimization of the use of limited network resources such as transmission bandwidth. To be accurate, digital video quality measurements must be based on the perceived quality of the actual video being received by the users of the digital video system rather than the measured quality of traditional video test signals (e.g., color bar). This is because the performance of digital video systems is variable and depends upon the dynamic characteristics of both the original video (e.g., spatial detail, motion) and the digital transmission system (e.g., bit rate, error rate). The goal of this report is to provide a complete description of the ITS video quality metric (VQM) algorithms and techniques. The ITS automated objective measurement algorithms provide close approximations to the overall quality impressions, or mean opinion scores, of digital video impairments that have been graded by panels of viewers.
\end{abstract}




\title{
The perfect ocean for drought
}

\author{
Martin P. Hoerling and Arun Kumar \\ NOAA/CIRES Climate Diagnostics Center
}

Science, 299: 691-694, 2003

\section{Significance}

The results described in this paper provide a new theory for the role of oceans in drought, and illuminate new avenues for long-lead prediction of drought. The results also reveal new evidence for an anthropogenic influence on climate at the regional scale. The warmth of Indo-west Pacific ocean temperatures that played a role in the 1998-2002 drought is consistent with the ocean's response to changing atmospheric chemistry, and was not solely due to natural variability. In this regard, the study is significant for identifying the atmospheric sensitivities to recent changes in tropical ocean states that can be capitalized upon for regional climate change projections in the 21 st Century.

This paper has been highly recognized by scientists and the popular media. It was highlighted in a non-technical article in Science by Richard Kerr, as well as in the interagency Climate Change Science Program's report of outstanding accomplishments to congress in "Our Changing Planet" and in the Encyclopedia Britannica's annual update on outstanding scientific advances.

\begin{abstract}
The 1998-2002 droughts spanning the United States, southern Europe, and Southwest Asia were linked through a common oceanic influence. Cold sea surface temperatures (SSTs) in the eastern tropical Pacific and warm SSTs in the western tropical Pacific and Indian oceans were remarkably persistent during this period. Climate models show that the climate signals forced separately by these regions acted synergistically, each contributing to widespread mid-latitude drying: an ideal scenario for spatially expansive, synchronized drought. The warmth of the Indian and west Pacific oceans was unprecedented and consistent with greenhouse gas forcing. Some implications are drawn for future drought.
\end{abstract}




\section{Author Index}

Akima, Hiroshi, 44

Alexander, Michael A., 140

Allan, David W., 29, 116

Allwine, Eugene J., 106

Anderson, David M., 169

Anderson, David N., 53

B

Baker-Jarvis, James, 122

Barger, Richard L., 52

Barnes, James A., 29

Barrick, Donald E., 68

Barsis, Albrecht P., 28

Bartram, Bruce, 163

Baugh, Kimberly E., 152

Bean, Bradford R., 32

Beehler, Roger E., 19

Benjamin, Stanley G., 126

Bergquist, James C., 162

Berry, Leslie A., 26

Blackmon, Maurice L., 135

Bradley, Raymond, 147

Brady, Charles H., 104

Brennan, J.A., 51

Brewster, Keith A., 126

Brümmer, Renate, 126

Bruno, Thomas J., 111

Buhr, Martin P., 106

Bussey, Howard E., 56

Butler, James H., 131

\section{C}

Caracena, Fernando, 63, 75

Case, Roslyn, 147

Chamberlain, George E., 91

Chappell, Charles F., 75

Childs, Greg E., 54

Chivers, Hugh J.A., 23

Churney, Kenneth L., 45

Clark, Alan F., 65, 66, 97, 108

Codrescu, Mihail V., 138

Cohen, Robert S., 48

Costanzo, Giovanni, 168

Crawford, Myron L., 55

Crow, Edwin L., 13

Cummings, Scott O., 131

Curtis, E.A., 162
Dally, James W., 129, 132

Danielson, Bruce L., 52, 91

Davies, Kenneth, 27

Davis, Dickie D., 116

Davis, Ethan R., 152

Day, Gordon W., 52, 91

Daywitt, William C., 92

Dean, J.W., 51

DeMarchi, Andrea, 168

Derr, Vernon E., 43

Deser, Clara, 135, 140

Diddams, Scott A., 162

Disney, Robert T., 57

Dlugokencky, Edward J., 128

Douglas, Maryanne, 147

Drullinger, Robert E., 73, 93, 162, 168

Dubé, William P., 145

Dutton, Evan J., 32

$\mathbf{E}$

Earnshaw, Kenneth B., 98

Eichenberger, Ali L., 155

Ekin, Jack W., 65, 66, 100, 110

Elkins, James W., 131

Elvidge, Christopher D., 152

Ely, James F., 86

Engen, Glenn F., 12, 76

Ericks, Lewis J., 54

Evans, David S., 58

Evans, Michael W., 68

Evenson, Kenneth M., 52, 67, 93

\section{F}

Fehsenfeld, Fred C., 33, 106

Ferguson, Eldon E., 33

Fickett, Fred R., 65, 66, 90

Finney, Bruce, 147

Fisher, Donald A., 131

Fitting, Dale W., 145

Flynn, Thomas M., 71

Franzen, Douglas L., 91

Frederick, Nolan V., 47, 61

Friend, Daniel G., 107

Fujita, Tetsuya T., 63

Fuller-Rowell, Tim J., 138

Fung, Inez, 123 


\section{G}

Gajewski, Konrad, 147

Gallagher, John G., 134

Gallawa, Robert L., 91

Garcia, Rolando R., 102

Gardner, Robert S., 13

Gilbert, Sarah L., 161

Goldfarb, Ron B., 124

Goodrich, Loren F., 90, 108

Goodwin, Robert D., 21

Green, John L., 60

Gupta, Anil K., 169

H

Hale, Paul D., 133

Hall, Bradley D., 131

Hall, John L., 52, 93

Hamilton, Clark A., 99

Hanley, Howard J.M., 86

Hardy, Douglas, 147

Haynes, William M., 61, 95, 151

Heavner, Thomas P., 168

Herbstreit, Jack W., 11

Hill, David A., 82, 153, 170

Hilton, Gene C., 146

Hiza, Michael J., 61, 95, 151

Hoer, Cletus A., 64, 76

Hoerling, Martin P., 149, 166, 172

Hollberg, Leo, 162, 168

Holloway, Christopher L., 170

Holt, John M., 156

Hord, Jesse, 87

Howard, Carleton J., 67

Hoxit, L. Ray, 75

Huber, Marcia L., 134

Hufford, George A., 88, 125

Hughen, Konrad, 147

Hurrell, James W., 166

I

Irwin, Kent D., 146

Itano, Wayne M., 142, 162

J

Jacoby, Gordon, 147

Jefferts, Steven R., 168

Jennings, Anne, 147

Jennings, Donald A., 31, 93

Jewett, Brian F., 126

Johnson, Victor J., 35
Kamper, Robert A., 49

Kanda, Motohisa, 81

Kaplan, Steven B., 65

Kasen, M. Bud, 62

Kautz, Richard L., 99

Keller, Mark W., 155

Kerns, David M., 85

Kihn, Eric A., 152

Kim, Ernest M., 91

King, Brian E., 142, 144

Kissick, William A., 88, 122, 165

Kneebone, C.H., 51

Kroehl, Herbert W., 152

Kropfli, R., 163

Ku, Harry C., 108

Kumar, Arun, 149, 172

$\mathbf{L}$

Lamoureux, Scott, 147

Lang, Patricia M., 128

Lasca, Andrew, 147

Lawrence, Robert S., 23

Layer, Howard P., 93

Ledbetter, Hassel M., 62

Lee, W. David, 162, 168

Leinbach, Harold, 16

Lelental, Mark , 124

Lemmon, John J., 167

Levi, Filippo, 168

Levine, Judah, 116

Liebe, Hans J., 112, 125

Little, C. Gordon, 16, 23, 37, 43

Liu, Shaw C., 106

Lloyd, Frances L., 99

Longley, Anita G., 28, 36, 88

Louie, Beverly, 101

M

Ma, Mark T., 59

MacDonald, Glen, 147

Maddox, Robert A., 75, 94

Magee, Joseph W., 151

Manabe, Takeshi, 125

Manahan, Michael P., 156

Mann, Douglas B., 51

Marks, Roger B., 127

Marsh, Francis J., 156

Martin, Russell C., 128

Martinis, John M., 137, 146, 155

Martner, Brooks, 163

Masarie, Kenneth A., 128

Matrosov, Sergey, 163

May, Peter T., 115

McCarty, Robert D., 50, 83, 87, 95

McColskey, J. David, 104 
McCowan, Chris N., 114, 136, 156

McHenry, Harry I., 62, 104

McLinden, Mark O., 134

Mechels, Steven E., 133

Meekhof, Dawn M., 142, 144, 168

Merritt, David A., 98

Middleton, David, 69

Mikesell, R.P., 62

Mockler, Richard C., 19

Moffett, R.J., 138

Monroe, Chris, 142, 144

Moore, Jay, 147

Moran, Kenneth P., 98, 115

Moreland, John, 100, 108

Morris, Derek, 56

Morrison, Graham, 134

\section{$\mathbf{N}$}

Nahman, Norris S., 72

Nahum, Michael, 137

Nelson, Craig W., 168

Nesenbergs, Martin, 74

Newell, Allen C., 109

Norton, Kenneth A., 15, 28

\section{O}

Oates, Chris W., 162

Olson, D.L., 114

Ott, Randolph H., 46

Overpeck, Jonathan T., 147, 154, 169

\section{$\mathbf{P}$}

Parker, Thomas E., 168

Parrish, David D., 106

Penland, Cécile, 143

Petersen, F. Russell, 52, 93

Peterson, Robert L., 110

Pinson, Margaret, 171

Pollock, Clifford R., 93

Powell, Robert L., 54, 65

Purtscher, Patrick T., 104

\section{Q}

Quegan, Shaun, 138

\section{$\mathbf{R}$}

Radebaugh, Ray, 34, 65, 101

Raffo, A.G., 131

Rainwater, James C., 107

Ravishankara, Akkihebbal R., 130

Read, David T., 65, 84, 104, 129, 132

Reed, Richard P., 62, 84, 97
Reinking, Roger, 163

Retelle, Michael, 147

Rice, Philip L., 28, 36

Roder, Hans M., 87

Rowland, F. Sherwood, 102

Ruth, Earl A., 156

Sardeshmukh, Prashant D., 143

Schlatter, Thomas W., 126

Schmeltekopf, Arthur L., 33

Scott, Russell B., 14

Seitz, Neal B., 113

Shelton, R.N., 108

Shirley, Jon H., 168

Shives, T. Robert, 104

Siewert, Thomas A., 114, 136, 145, 156

Smith, David R., 101

Smith, Ernest K., Jr., 10

Smith, Sheldon, 147

Smith, Tracy L., 126

Snider, Charles S., 19

Solomon, Susan, 102, 130

Spaulding, Arthur D., 57, 69

Spies, K.P., 24

Stamus, Peter A., 126

Steele, L. Paul, 128

Strauch, Richard G., 98, 115

Sugar, George R., 25

Sullivan, Donald B., 116

Svoronos, Paris D.N., 111

Swann, William C., 161

Swanson, Thomas H., 131

Takahashi, Taro, 123

Tans, Pieter P., 123, 128

Thompson, Curt A., 124

Thompson, Moody C., 11

Thompson, Thayne M., 131

Timmerhaus, Klaus D., 20

Trainer, Michael, 106

Turnipseed, Andrew A., 130

Udem, Thomas, 162

Utlaut, William F., 48

V

van de Kamp, Douglas, 98

VanZandt, Thomas E., 60

Vanzura, Eric J., 122

Vogel, K.R., 162 
Vogler, Lewis E., 89

Voran, Stephen, 157

W

Wait, James R., 22, 24, 82

Walls, Fred L., 73, 168

Warnock, James M., 60

Warren, Rachel F., 130

Weber, Bob L., 68

Weber, Lloyd A., 50

Weintraub, Stanley, 10

Weiss, Marc, 116

Wells, Joseph S., 52, 93

Wepman, Jeffery A., 141

West, E. Dale, 45

Westberg, Halvor H., 106

Williams, Dylan F., 127

Williams, Eric J., 106

Wilson, Perry F., 170

Wineland, David J., 73, 142, 144

Winkler, Richard H., 60
Wolf, Stephen, 171

Wolfe, Alex, 147

Wollman, David A., 146

Woodhouse, Connie A., 154

Wuebbles, Donald J., 102

$\mathbf{X}$

Xu, Taiyi, 166

Y

Young, Matt, 91, 133

$\mathbf{Z}$

Zaltsman, E.B., 56

Zhong, Min, 149

Zielinski, Greg, 147

Zimmerman, James E., 47, 49, 71, 101

Zimmerman, Neil M., 155 


\section{Subject Index}

\section{A}

absolute thermometer, 49

absorption

cell (calorimeter), 31

earth-space radio propagation, 32

millimeter-wave propagation model (MPM), 112

riometer, 16

accuracy, atomic clock, 168

acoustic radar

clear atmosphere, 43

development, 37

Radio Acoustic Sounding System, 115

Advanced Weather Interactive Processing System

(AWIPS), 8

Aeronomy Laboratory (NOAA), 6, 7, 33, 60, 98, 115

afterglow reaction, 33

air

conditioners, chlorofluorocarbons, 131, 134

quality, 106

aircraft

accidents, 63

icing, 163

observations, 126

Aircraft Communications Addressing and

Reporting System (ACARS), 126

Alaska, 11

Allan variance, 29

Allardice, John F., 3

All-Union Scientific-Research Institute of PhysicoTechnical and Radio-Technical Measurements (VNIIFTRI) (USSR), 56

alternating-field susceptometry, 124

American National Standard, 8

American Physical Society, 108

American Society for Testing and Materials (ASTM)

Committee on Superconductivity, 65

E23, Standard Test Methods for Notched Bar Impact Testing of Metallic Materials, 156

superconductor measurement standard, 90

American Welding Society, 114

amine-absorber pressure vessel, 104

analog-to-digital converters (ADCs), 141

Anger function, 81

anisotropic ionosphere, 26

anomalies

Appleton anomaly, 53

North Atlantic Ocean, 135

ocean mixed layer, 140

rainfall, 149

sea surface temperature growth, 143

Antarctic ozone hole, 7, see also ozone antenna scattering-matrix, see PWSM

antenna-antenna interactions, 85

antennas

arrays, 59

calibration, 81

directivity, 28

microwave, 85

system loss, 15

anthropogenic influence

chlorine compounds, 102

drought, oceans, 172

fluorinated gases, 130

methane, 128

non-methane hydrocarbons, 106

North Atlantic climate change, 166

refrigerants, 134

smog, 106

anvil cloud, 94

Apollo 11 mission, 5

Appleton anomaly, 53

apportionment boundaries, 113

Arctic, 147

area prediction mode, 88

arrays, antenna, 59

Asian monsoon, 169

ASME Boiler and Pressure Vessel Code, 114

atmosphere

lifetimes (chemistry), 130

noise, 27

radio noise, 167

radio spectrum absorption, 112

radio waves, 32

refractive index, 10

refractivity variations, 11

residence, 130

turbulence, 11

Atmospheric General Circulation Model (AGCM), 149

atomic clocks, see also NIST F1

accuracy, 168

Allan variance, 29

laser-cooling, 73

laser-line narrowing, 7

NBS-1, 5

NBS-2, 19

optical, 162

atomic frequency standards, 29

attenuation

ground waves, 26, 82

knife-edge diffraction, 89

long-term median values, 28

optical fiber measurement systems, 91

radio signal, 32

rates, 24 
attenuation-continued

stratified media models, 22

waves, irregular terrain, 46

audio quality, 8

auroral

physics, 58

zone, 16

austenitic stainless steel, 84, 114, 136

Automated Computer Time Service (ACTS), 116

Aviation Weather Center, 126

\section{B}

backscatter, 91

Bakker, Robert, 7

balloon-borne instruments, 60

balloonsonde ozone data, 102

bandwidth, 91

Big Thompson Storm, 75

bioelectromagnetic effects, 81

Blue Planet Prize, 8

bolometer mount, 12

Boltzman's constant, 49

Bose-Einstein Condensate, 8, 73

Boulder Chamber of Commerce, 2

Boulder Elks Lodge, 3

break junctions, 100, 108

British Antarctic Survey, 102

broadband

antennas, 59

model, 112

six-port network analyzer, 64

business noise, 57

\section{C}

calibration

cryogenic flow, 51

dual six-port network analyzer, 64

Thru-reflect-line (TRL), 127

transverse electromagnetic (TEM) cells, 55

vector network analyzers, 76

calorimeter

design, 45

measurement, 31

Canada, 11

capacitance standards, 137,155

carbon

dioxide (CO2)

anthropogenic effects, 128

Mauna Loa observatory, 5

sources and sinks, 123

emissions, 152

cardiography, 47

Carnahan-Starling-Desantis (CSD) equation of state, 134

Carter, Jimmy (President), 6 cavity resonators, 56

CCIR/ITU-R model, 7

CCITT, see ITU-T Recommendations

cellular telephones, 141

Central Radio Propagation Laboratory (CRPL),

$1-3,5,6,10,11,15,23,27,32$

ceramic superconductors, 110

cesium, 6, 8, 30, 168

channel systems, 28

Charpy impact test, 156

chemical analysis, 111

Cheyenne Mountain, 11

chlorine compounds, 102

chlorofluorocarbons (CFCs)

atmospheric lifetimes, 130

growth rates, 131

reaction rates, 67

refrigerant database (REFPROP), 134

chromatography, 111

city lights, 152

cladding diameter, 133

clear air

Doppler radar, 115

millimeter-wave propagation model, 112

remote sensing techniques, 43

climate

Arctic, 147

change

carbon dioxide, 123

drought, 154, 172

fluorinated gases, 130

methane, 128

North Atlantic, 166

North Atlantic Ocean, 135

reconstructions, 169

Climate Diagnostics Center (CDC), 8

Climate Monitoring and Diagnostics Laboratory (CMDL), 5, 7, 128, 131

climatic time scale, 32

clocks, see also atomic clocks, NIST-F1

cesium, 8, 168

optical, 162

performance, 29

time synchronization, 116

clouds

detection, 152

radio signal attenuation, 32

winter, 163

coherent reception, 69

cold core system (meteorology), 94

Colorado National Guard Armory, 3, 5

Colorado wind-profiling network, 99

Columbia Space Shuttle, 8

commercial flight planning, 126

communication systems

radio, 27

UWB effects, 165 
Comprehensive Ocean-Atmosphere Data Set

(COADS), 135

computer clocks, 116

Computer Method, 44

Condon, Edward U., 2

confidence

belt, 13

interval, 13

constants (mathematics), 10

contact micrometer, 133

Continental flight 426, 63

controlled-NOT, 142

convection

downbursts, 63

floods, 75

tropical, 94

cooling system designs, 134

Cooperative Institute for Research in Environmental Sciences (CIRES), 6

coplanar waveguide, 127

Cornell, Eric, 8

cosmic noise, 16

Crawford Cells, 55

critical current, 66, 90

cryocoolers, 71, 101

cryogenic

equipment design, 54

noise, 92

testing, 84

welding, 136

Cryogenic Data Center, 35

Cryogenic Engineering Conference (CEC), 20, 101

Cryogenic Engineering Laboratory (CEL), 3, 14

Cryogenic Flow Research Facility, 51

Cryogenics Division, 65, 66, 95, 97

cryogens, 83

cumulus convection, 63

curved earth, 24

customer behavior, 74

\section{D}

Davis, Dick, 6

Debye model, 72, 125

decadal

climate change, 166

variability, 135

decentralization of the Federal government, 2

Defense Meteorological Satellite Program (DMSP), 152

demagnetization, 124

density

liquified natural gas, 61

measurements, 95

metrology, 21

depolarization ratio, 163

device testing, transverse electromagnetic (TEM), 55 diabatic heating, 94

dielectric

complex permittivity, 125

properties, 55

digital

devices, reduced test times, 170

signal processing, 141

speech, 157

system performance, 69

television, 36

terrain elevation databases, 36

video quality, 171

dilution refrigerator, 34

dinosaur Drinker nisti, 7

dipole probes, 81

directivity, unintentional emitter, 170

discharge-flow system, 67

distance

measures, 157

propagation characteristics, 26

Distant Early Warming (DEW), 11

Ditsch, Carl, 6

Doppler

effect, 60

radar, 115

wind profilers, 7, 98

downbursts, 63

drawing smooth curves, 44

drift, 68

drizzle, 163

drought, 154, 172

Drinker nisti, 7

Drullinger, Robert E., 7

dual six-port network analyzer, 64, 76

dual-polarization radar, 163

\section{$\mathbf{E}$}

Earth

curvature, 26

rotation, 19

stratified media models, 22

earth-ionosphere waveguide, 24

earth-space radio propagation, 23

Echo Lake Cosmic Ray Symposium, 2

echo-sounders, 37

economic impact, 116

ecosystem alteration, 147

EEEL Outstanding Authorship Award, 170

Eisenhower, Dwight D. (President), 4

El Niño Southern Oscillation (ENSO)

growth, 143

North Atlantic climate effects, 166

prediction, 8

teleconnection patterns, 149 
electric

current standards, 137

lengths, 11

noise, 49

electromagnetic

interference, 170

radiation hazard meters, 55

waves, stratified media, 22

electromagnetic compatibility (EMC)

reverberation chambers, 153

short dipole probes, 81

TEM cells, 55

electron

beam moiré, 132

counting, 155

precipitation, 58

pumps, 137

electron-layer production, 27

electron-tunneling, 100

electrothermal feedback, 146

emissions

electrically large sources, 170

perfluorocarbons (PFCs), 130

reverberation chambers, 153

Emmy Award, 6

empirical orthogonal function (EOF), 135

end-to-end virtual connections, 113

energy

companies, 95

gap, 100, 108

Engen calorimeter, 12

Environmental Data Service (EDS), 6

Environmental Science Services Administration (ESSA), 4, 5, 6

Environmental Technology Laboratory (ETL), 7, 8, $68,115,163$

equatorial

anomaly, see Appleton anomaly

F-region, 53

Erlang formulas, 74

error

analysis techniques, 109

bars, 13

ESSA Research Laboratory (ERL), 4, 6, 7

Eurasian snow cover, 169

Evanson, Ken, 7

excitation factors, 24

explosions, 104

\section{F}

Faller, James, 6

Faraday rotation, 23

far-field radiation patterns, 91

Federal Aviation Administration (FAA), 126, 163

Federal Communications Commission (FCC), 6

Fermi-Dirac gas model, 34 ferrite, 114

Filla, Jim, 7

filler metals, 136

fine structure constant, 155

fixed-station military radios, 141

flash floods, 75

flask sampling, 128

flicker noise, 29

flow loop, 51

fluid property measurement, 21, 61, 83

Fluids Pack, 83

fluorinated gases, 130

FM broadcasts, 15

fog, 32

Forecast Systems Laboratory (FSL), 7, 8, 60, 98

forecasts

Colorado wind-profiling network, 98

El Niño Southern Oscillation, 149

flash floods, 75

mesoscale convective complexes, 94

Rapid Update Cycle, 7

severe storm, 7

short-term, 126

wind profilers, 60

FORTRAN, 83

fossil Globigerina bulloides, 169

fracture mechanics, 62

F-region, 53

frequency

human-made radio noise, 57

ionospheric effects, 23

prediction, 27

Front Range (Colorado), 60, 75

G

Gaussian

hill, 46

noise, 69

General Circulation Model (GCM), 123

geomagnetic

fields, 27, 53

storms, 16,138

glacial melting, 147

glass beams, 100

Glaze, David J., 7

global

carbon cycle, 123

distance communication, 22

warming, 147, 169

Global Assimilation of Ionospheric Measurements

(GAIM) model, 53

Global Warming Potential (GWP) index, 130

gradiometer, 47

grain-boundary weak links, 110

granular superconductors, 110

grating, 132 
gravity waves, 138

greenhouse gas forcing, see anthropogenic influence greenhouse gases, see specific type (e.g., methane)

Greenland, 11

ground waves

attenuation, 26

transmission loss, 15

group-path delay, 23

Guinness Book of World Records, 7

\section{H}

Haar-Gallagher-Kell equation of state, 134

halogen gases, 130, 131

heaters (high-power transmitters), 48

helium liquids, 34

heterogeneous chemistry, 102

High Altitude Observatory, University of Colorado, 2

high critical temperature superconductor (HTS), 108

high-energy

pulsed Doppler lidar, 7

x-ray diffraction, 145

high-field magnets, 67

high-frequency (HF)

antenna arrays, 59

atmospheric radio noise, 167

ionospheric beams, 48

ionospheric propagation, 27

High-Frequency Active Auroral Research Program (HAARP), 48

High-Power Auroral Stimulation (HIPAS), 48

high-temperature superconductors (HTS), 110

human

health, 106

perception, 171

made, see anthropogenic influence

humidity, 32

hydrocarbons, 106

hydrogen, 87, 104

hydrometeors, 163

hydroperoxyl radicals, 67

\section{I}

ice

clouds, 102

cores, 128

crystals, 163

IEEE EMC Transactions Prize Paper Award, 170

immunity measurements, 153

impedance, 76, 127

impulsive interference, 69

incoherent reception, 69

Indo-Pacific Ocean, 143, 166, 172

Industrial Revolution, 128

inspector training, 104
Institute for Telecommunication Sciences (ITS),

$$
6,7,8
$$

Institute for Telecommunication Sciences and

Aeronomy (ITSA), 4, 5, 6

Institutes for Environmental Research, 6

instrumentation, chemical, 111

integral equation, radio wave propagation, 46

Integrated Services Digital Network (ISDN), 113

interference

impulsive, 69

satisfactory service requirements, 28

intergranular Josephson junctions, 110

internal climate system, 147

International Cryocooler Conference (ICC), 71

International Electrotechnical Commission (IEC), 90

International Geophysical Year (IGY), 5, 27

International Organization for Standardization (ISO), 65,134

International Radio Consultative Committee (CCIR), 57

International Reference Ionosphere (IRI), 138

International Year of the Quiet Sun, 27

Internet Protocol (IP) networks, 113

Internet Time Service (ITS), 116

interpolation, mathematical method, 44

ionosphere

absorption, 16

chemistry, 33

F2 region, 53

geomagnetic storms, 138

layered model, 24

meteor trails, 25

radio wave effects, 23

radio wave modification, 48

radio wave propagation, 27

reflection coefficient, 26

stratified media models, 22

wave hop theory, 26

Iowa State University, 108

irregular terrain integral equation, 46

Irregular Terrain Model (ITM)

point-to-point methods, 28

tropospheric radio transmission loss, 36

user's guide, 88

isentropic analysis, 126

isochoric gas expansion, 151

isoperibol calorimetry, 45

ITS Irregular Terrain Model (ITM), see Irregular Terrain Model

ITU-T Recommendations, 113

\section{$\mathbf{J}$}

Jennings, Donald, 5

Jesperson, James, 6

John F. Kennedy International Airport, 63

Johnson noise, 49 
Joint Institute for Laboratory Astrophysics (JILA), $4,6,8$

Josephson

Effect, 49

phenomena, 65

voltage standard, 99

Junction Model, 110

\section{$\mathbf{K}$}

Ka-band radar, 163

Kallistratova, M.A., 37

Kamas, George, 6

Ketterle, Wolfgang, 8

kinetic schemes, 67

knife-edge diffraction, 89

krypton, 93

\section{$\mathbf{L}$}

La Niña, see El Niño Southern Oscillation

Labrador Sea, 135

land mobile radio (LMR), 8

land-mobile system applications, 88

Large Coil Project, 84

large-scale convection, 94

laser

cooling

clocks see atomic clocks

origination, 73

quantum logic gate, 142

superposition, 144

energy standard, 31

measurements

calorimeter, 45

methane-stabilized, 52

speed of light, 93

laser-magnetic resonance detection, 67

length measurement, 122

lidar, 7, 43

linchpin reaction, 67

linear arrays, 59

liquefied natural gas (LNG), 61, 95

liquid

density measurements, 95

helium, 34, 71

hydrogen, 3,5

methane, 61

nitrogen, 51, 92

liquification of gases, 14

listening tests, 157

Little Ice Age, 147

local time, 138

locally optimum Bayes detection (LOBD) receivers, 69

log-periodic dipole array, 59 long distance

communication, 25

propagation, 26

longitude

dependence, 138

variability, 53

Longley-Rice Model, see Irregular Terrain Model

Lorentzian function, 125

loss measurements, 56

lossy ground, 59

low latitude ionosphere, 53

low temperature measurements

materials, 97

vapors, 107

Lowe, John P., 7

lower atmosphere

probing, 37

radio wave propagation, 32

low-frequency radio waves, 26

\section{M}

magnesium ions, 73

magnetic

latitude, 53

susceptibility, 124

suspension densimeter, 61, 95

field

aligned potential difference, 58

Earth, 24

Josephson junctions, 110

weak, 47

Magnetic Fusion Test Facility (Lawrence Livermore Laboratory), 84

magneto-ionic theory, 27

magnetometer, 47

magnetospheric energy, 138

magnets, 7, 84

mapping

city lights, 152

ocean surface currents, 68

"March Meeting," American Physical Society, 108

Marconi, Guglielmo, 2

Markov model, 74

materials measurements, 97

Materials Reliability Division, 8

Materials Research Society, 108

Mauna Loa Observatory (Hawaii), 5

McKay Helm Award, 114

mechanical stirring, 153

megadrought, 154

Meissner effect, 124

mercury ions, 162

Mesometeorological Group, 75

mesonetwork, 63

Mesoscale Analysis and Prediction System (MAPS), 126 
Mesoscale Convective Complexes (MCCs), 94 metal castings, 145

meteor trails, 25

meteorology, radio, 32

meter definition, 7, 52, 93

methane

greenhouse gas, 128

liquefied natural gas densities, 95

magnetic suspension densimeter, 61

thermodynamic and transport properties, 83

methane-stabilized laser, 52

metrological accuracy, 137

microbursts, 63

microcalorimeter, 12,146

microelectronic devices, 129

micromechanics, 132

microwave

antennas, 85

microcalorimeter, 12

network analyzer, 64

techniques, 10

military applications

Allan variance, 29

area predictions, 88

fixed-station radios, 141

high frequency bands, 167

reverberation chamber measurements, 153

transhorizon communications, 11

VLF radio wave propagation, 24

millimeter-wave

bands, 5

propagation model (MPM), 112, 125

minimum scattering antennas, 85

mixed layer, ocean, 140

modal interference, 143

mode theory, 153

model interference, 69

Modified Bennedict-Webb-Rubin (MBWR) equation of state, 134

moire, 132

monopulse array, 59

monsoon, 169

Montreal Protocol, 131

Mount Washington Icing Sensors Project (MWISP), 163

MPM, see millimeter-wave propagation model

multi-service networks, 74

$\mathbf{N}$

National Aeronautical and Space Administration (NASA), 8

National Association of Securities Dealers (NASD), 116

National Geophysical Data Center (NGDC), 6

National Medal of Science, 102

National Meteorological Center (NMC), 126
National Ozone Expedition, 7, 102

National Research Council, Canada, 56

National Transportation Safety Board (NTSB), 63

National Weather Service (NWS), 7, 98

modernization, 8

natural

gas properties, 151

hydrocarbons, 106

NBS-1, 5, see also NIST-F1

NBS-2, 19

National Bureau of Standards (NBS) Tech Note 101, $15,28,36$

$\mathrm{Nb}-\mathrm{Sn}$ filament, 100

near-field

measurements, 85

radiation patterns, 91

near-infrared (NVIR) radiance, 152

negative ions, 33

network

analyzer, 64

congestion, 74

neural activity, 47

Newton-Raphson iteration, 81

Nicolson-Ross method, 122

nighttime lights, 152

National Institute of Standards and Technology (NIST)

TN 1500-3, 145

NIST-7, international time standard, 7

NIST-F1, see also atomic clocks

accuracy, 168

atomic beam frequencies, 19

cesium fountain clock, 8

optical clock, 162

radiation-pressure cooling, 73

nitrogen

flow loop, 51

liquefied natural gas densities, 95

magnetic suspension densimeter, 61

nitrogen

noise standard, 92

strengthened austenitic stainless steels, 84

thermodynamic and transport properties, 83

oxide

hydroperoxyl radicals reaction, 67

smog, 106

National Oceanic and Atmospheric Administration (NOAA)

Profiler Network, 60

Unit Citation award, 75

Nobel Prize, 4, 8, 73, 100, 102, 108

nocturnal events, 94

noise

atmospheric, 27

flicker, 29

human-made, 57

radio, 167

rural, 57 
noise-continued

standards, 92

thermometry, 49

wideband, 16

non-methane hydrocarbons (NMHCs), 106

nonpolar fluid mixtures, 86

normalizing blocks, 157

North American Carbon Cycle Research Program, 123

North Atlantic Ocean, 135

North Atlantic Oscillation (NAO), 166

notched bar impact testing, 156

n-port devices, 76

\section{$\mathbf{O}$}

oblique propagation, 27

Occupational Safety and Health Administration (OSHA), 104

ocean

carbon dioxide sink, 123

mapped surface currents, 68

mixed layer temperature anomalies, 140

observations, 8

role in drought, 172

warming, 166

ocean-atmosphere interaction, 135, 143

Office of Naval Research (ONR), 71

on-wafer transmission lines, 127

Operational Linescan System (OLS), 152

optical

clocks, see atomic clocks

communications, wavelength standards, 161

fiber characterization, 91

fiber geometry, 133

frequency "comb", 162

pulse waveforms, 72

remote sensing, 43

orographic precipitation, 75

orthobaric liquid density measurements, 95

oscillators, 29

oxide barriers, 100

oxygen, 50

ozone

alternative refrigerants, 134

Antarctic depletion, 102

depleting substances, 131

layer protection, 7

production, 67

rural, 106

smog, 106

\section{$\mathbf{P}$}

packet transmission, 25

packet-switched services, 113

paleoclimate data, 147, 154 parahydrogen, 87

PCS base stations, 141

pendulum impact test, 156

perceptual transformations, 157

perfluorocarbons (PFCs), 130

performance standards, 113

permittivity

international intercomparison, 56

transmission line, 122

water, 125

peroxyacetyl nitrate (PAN), 106

Peterson, Elmore, 3

phase velocities, earth-ionosphere, 24

Philadelphia International Airport, 63

phonon coupling, 100

photochemistry, 106

picosecond time-domain measurements, 72

piecewise function, 44

piezometer, 21

pipeline girth welds, 62

Pitzer's acentric factor, 86

planar near-field

measurements, 109

scanning theory, 85

plane-wave

integral representation, 153

spectra, 85

plasma, ionospheric, 27,48

Platteville, Colorado, 48

point-to-point scatter systems, 15

Poisson variable, 13

polar regions, 102

polarization changes, 23

pollution, air, 106

polynomials, 44

population, 152

potential

difference, 58

temperature, 126

practical superconductors, 65,90

precipitation, 75

prediction

climate change, 128

communication system behavior, 167

communication systems, 32

drought, 172

HF ionospheric propagation, 27

liquefied natural gas densities, 95

long-term median transmission loss, 36

transmission loss, 28

viscosity, 86

pressure vessels, 104

probe calibration, 55

Program for Regional Observing and Forecasting Systems (PROFS), 7

propagation

acoustic waves, 115 
propagation-continued

constant measurement, 127

effects of cloud droplets, 125

ground waves, 82

irregular terrain, 46

long distance, 26

meteors, 25

millimeter-wave propagation model (MPM), 112

modeling, 15

oblique, 27

radio wave system loss, 15

theory, 22

properties

cryogens, 83

hydrogen, 87

materials, 97

natural gas, 151

thin films, 129

propulsion systems, 50

pseudo-Brewster angle, 26

public safety, 141

pulse measurements, 71

pulse tube refrigerators, 101

pulsed laser, 31

PVT (pressure-density-temperature), 21, 86

PWSM theory, 85

\section{Q}

quality factor (Q), 153, 170

Quality of Service (QoS), 113

quantum

computing, 142

superposition theory, 144

quartz oscillators, 19, 29

quasiparticle tunneling, 108

qubits (quantum bits), 142

\section{$\mathbf{R}$}

radar

acoustic, 37, 43

aircraft safety, 63

clear air Doppler, 115

Colorado wind-profiling network, 98

depolarization, 163

mesoscale analysis, 75

ocean surface currents mapping, 68

UWB signal effects, 165

VHF, 60

radiated power, 170

radiation

hazard monitoring, 81

pressure cooling, 73

Radio Acoustic Sounding System (RASS), 115 radio

communications, 27, 33

frequency

atmospheric refractive index, 10

spectrum efficiency, 28

horizon, 11

noise

high-frequency atmospheric, 167

human-made, 57

paths, 28

propagation

ITS/Longley-Rice model, 88

meteor trail reflection, 25

prediction, 28

receivers, 141

refractive index of air, 32

signal attenuation, 88

spectrum, 165

system

design, 36

satisfactory service requirements, 28

transmission, 25

waves

attenuation, 46

high-frequency, 27

long, 26

lower atmosphere propagation, 32

meteor interaction, 25

phase measurements, 11

scattering, 11

system loss propagation, 15

versus acoustic, 37

VLF, 24

Radio Laboratory Section, 2

Radio Propagation Executive Council, 2

Radio Spectrum Measurement System (RSMS), 7

radiographs, 62

radiosondes, 115

Rapid Update Cycle (RUC), 7, 126

rawinsondes, 60, 126

reaction rates, chlorofluorocarbons, 67

received signals, 25

receivers, 141

receiving systems, 69

reduced test times, 170

reemergence mechanism, 140

reference plane position, 122

refineries, 104

reflection

height propagation characteristics, 26

meteor trails, 25

reflectometers, 64

reflectors, moon, 6

refraction, ionospheric radio waves, 23

refractivity, air, 10, 32

refrigerants (chlorofluorocarbons), 131, 134 


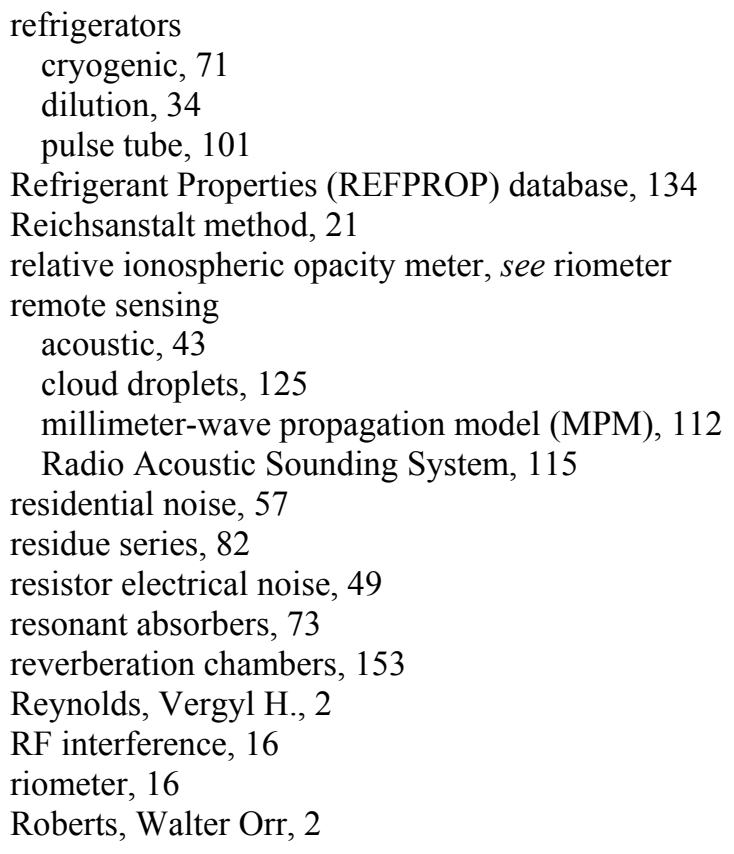

S

Shelkunoff, S.A., Award, 109

Satellite Home Viewer Improvement Act, 36

scanning

confocal microscope, 133

electron microscopy (SEM), 132

scattering, permittivity determination, 122

scattering-matrix theory, 85

Schrödinger cat, 144

scintillations, ionospheric, 23

sea surface temperature (SST)

deep mixed layer anomalies, 140

ENSO teleconnection phases, 149

North Atlantic climate change, 166

sea surface temperature (SST)

North Atlantic Ocean anomalies, 135

optimal anomaly growth, 143

role in droughts, 172

second definition (time), 6

sensing applications, 165

service system models, 74

severe storms, 7, see also Big Thompson, mesoanalysis

shadow region, 26

Shirley, Jon H., 7

short dipole probes, 81

short-term forecasts, 126

short-waves (meteorology), 94

Siegwarth, Jim, 7

signal detection, 69

sinks, carbon dioxide, 123 six-port

reflectometers, 64

systems, 76

slush hydrogen, 6

small curvature approximation, 82

smog, 67, 106

smooth curve fitting, 44

sodars, 37

software defined radio (SDR), 141

solar

flares, 16

irradiance, 147

solid materials, themal conductivity, 54

solidification models, 145

Solomon, Susan, 8

source-sink distributions, 123

Space Age, 27

space applications, 101

Space Disturbance Forecast Center, 6

Space Environmental Forecasting Division, 6

Space Shuttle Columbia, 8

S-parameters, 127

spearhead echo, 63

specific heat of fluids, 21

spectroscopy, 111

spectrum

efficiency, 28

management, 165

speech quality, 157

speed of light, 52, 93

stable linear dynamical system, 143

stainless steel, 84, 114, 136

Standard Reference Materials

\# 2520, 133

wavelength standards, 161

Stapleton International Airport (Denver), 63

statistics

atomic frequency standards, 29

immunity and emission measurements, 153

stochastic forcing, 143

storm damage, 63

Storm Prediction Center (SPC), 126

storms, upper atmosphere, 138

stratified media, 22

stratosphere

chlorofluorocarbons, see chlorofluorocarbons

ice clouds, 102

ozone layer, see ozone

wind profiling radar, 60

stress, superconducting wires, 66

submarines, 26

sunlight

geomagnetic storms, 138

smog production, 67, 106

Sunset radar, 7, 60 
superconducting

amplifier, 49

devices, small, 71

energy gap, 108

measurement standard, 90

wires, 66

Superconducting Quantum Interference Device

(SQUID), 47

superconductors

alternating-field susceptometry, 124

critical current, 90

practical, 65

tunneling spectroscopy, 100

supercurrents, 110

superposition theory, 144

surface

currents, ocean, 68

impedance, 82

surface-level ozone, 106, see also ozone

susceptometry, 124

switched telecommunication networks, 74

synchronization, time, 116

synoptic time scale, 32

system loss, 15

\section{$\mathbf{T}$}

Table of Allotments, 36

Tech Note 101 (NBS), 15, 28, 36

telecommunications

Allan variance, 29

atmospheric radio waves, 33

human-made radio noise, 57

performance measurement, 13

teleconnections, 149

telephone networks, 74

television relay, 15

TEM, see transverse electromagnetic

temperature measurements

Arctic warming, 147

Radio Acoustic Sounding System, 115

Terminal Doppler Weather Radar, 63

terrain, see irregular terrain

thermal conductivity, 54, 107

Thermal-energy associative-detachment reactions, 33

thermistor detectors, 76

thermoacoustic refrigerators, 101

thermocline, 140

thermometers, 49, 146

thermophysical properties, oxygen, 50

thermosphere, 138

Thévenin's equivalent circuit, 81

thin films, 129

Thru-reflect-line (TRL)

calibration, 127

method, 76

Thru-Short-Delay (TSD), 76 thunderstorms, 94, see also convection, severe storms, mesoscale convective complexes

time

measurements, 29

synchronization, 116

trace gases

Arctic climate change, 147

methane, 128

smog, 106

Trans-Alaska pipeline, 62

transhorizon communications, 11

transmission

lines

impedance standards, 76

on-wafer, 127

permittivity, 122

loss

concept development, 15

tropospheric communication circuits, 28

paths, 11

Transmission/Reflection Method, 122

transmitted speech quality, 157

transmitters, ultrawideband, 165

transport critical-current, 110

transverse electromagnetic (TEM) cells, 55

trapped surface waves, 82

traveling-wave antennas, 59

tropics

convective systems, 94

El Niño Southern Oscillation phases, 149

North Atlantic climate effects, 166

role in droughts, 172

sea surface temperature growth, 143

troposcatter, 11, 15

troposphere

acoustic remote sensing, 37

communication circuits, 28

radio propagation, 15

radio transmission loss, 36

remote sensing, 42

stratified media models, 22

winds, 60, 98

Tropospheric Propagation Section (CRPL), 11

tropospheric scatter, see troposcatter

Truman, Harry S., 2

tunnel junction measurements, 100

tunneling spectroscopy, 100, 108

turbulence, atmospheric, 11

TvTime, 6

\section{$\mathbf{U}$}

U.S. Capitol Dome, 8

ultra high frequency fields, 11

ultrawideband (UWB), 8, 165

unintentional emitter, 170

Union Oil Company, 104 
universal quantum logic gate, 142

Universal Time (UT), 138

University of Colorado (CU), 5, 6

urban sprawl, 152

USAF Air Weather Service, 6

user behavior, network congestion, 74

\section{V}

very high frequency (VHF)

Colorado wind-profiling network, 98

ionospheric soundings, 5

stratified media models, 22

troposcatter, 11

wind profiling radar, 60

very low frequency (VLF)

ionospheric soundings, 5

long distance propagation, 26

radio wave propagation, 24

stratified media models, 22

Video Quality Metric (VQM), 8, 171

virial coefficients, 107

visible radiance, 152

volcanic activity, 147

voltage

deviation, 167

measurement, 99

\section{W}

W.R.G. Baker Award, 109

warm core system (meteorology), 94

wave hop theory, 26

Wave Propagation Lab, 60

waveform model, 167

wavelength-division-multiplexed (WDM) optical communications systems, 161

weak links, high-temperature superconductors, 110

Weather Bureau, 6, see also National Weather Service welding

austenitic stainless steels, 84

ferrite content, 114

pipeline girth, 62

repair, 104

structures at cryogenic temperatures, 136

White House, 6

white-light interference microscope, 133 wideband

model, 167

noise, 16

Wieman, Carl, 8

wind

damage footprints, 63

geomagnetic storms, 138

monsoonal, 169

neutral, 53

profilers

buoy-mounted, 8

Colorado wind-profiling network, 98

high-energy pulsed Doppler lidar, 7

Mesoscale Analysis and Prediction System, 126

Space Shuttle Columbia, 8

Sunset VHF radar, 7, 60

Wind Profiler Demonstration Network, 115

shear, 63

winter season

El Niño Southern Oscillation events, 149

ice shapes in clouds, 163

North Atlantic climate variations, 135

ocean mixed layer anomalies, 140

wireless systems, 165

World Data Center (WDC), 5

World Trade Center, 8

World War II Interservice Radio Propagation

Laboratory (IRPL), 27

WR10 thermal noise, 92

Wullenweber antenna, 59

\section{$\mathbf{X}$}

X.25/X.75 Protocols, 113

$\mathrm{X}$-band, 12

$\mathrm{x}$-ray

detection, 146

diffraction, 145

Yagi-Uda antennas, 59

Yeager, James J., 3 